\title{
Field-Theoretic Weyl Deformation Quantization of Enlarged Poisson Algebras ${ }^{\star}$
}

\author{
Reinhard HONEGGER, Alfred RIECKERS and Lothar SCHLAFER \\ Institut für Theoretische Physik, Universität Tübingen, \\ Auf der Morgenstelle 14, D-72076 Tübingen, Germany \\ E-mail: reinhard.honegger@uni-tuebingen.de, alfred.rieckers@uni-tuebingen.de, \\ lothar.schlafer@uni-tuebingen.de
}

Received December 20, 2007, in final form May 06, 2008; Published online May 29, 2008

Original article is available at http://www.emis.de/journals/SIGMA/2008/047/

\begin{abstract}
C^{*}$-algebraic Weyl quantization is extended by allowing also degenerate presymplectic forms for the Weyl relations with infinitely many degrees of freedom, and by starting out from enlarged classical Poisson algebras. A powerful tool is found in the construction of Poisson algebras and non-commutative twisted Banach-*-algebras on the stage of measures on the not locally compact test function space. Already within this frame strict deformation quantization is obtained, but in terms of Banach-*-algebras instead of $C^{*}$-algebras. Fourier transformation and representation theory of the measure Banach-*algebras are combined with the theory of continuous projective group representations to arrive at the genuine $C^{*}$-algebraic strict deformation quantization in the sense of Rieffel and Landsman. Weyl quantization is recognized to depend in the first step functorially on the (in general) infinite dimensional, pre-symplectic test function space; but in the second step one has to select a family of representations, indexed by the deformation parameter $\hbar$. The latter ambiguity is in the present investigation connected with the choice of a folium of states, a structure, which does not necessarily require a Hilbert space representation.

Key words: Weyl quantization for infinitely many degrees of freedom; strict deformation quantization; twisted convolution products on measure spaces; Banach-*- and $C^{*}$-algebraic methods; partially universal representations
\end{abstract}

2000 Mathematics Subject Classification: 46L65; 47L90; 81R15

\section{Introduction}

In the present investigation we elaborate a kind of field theoretic Weyl quantization, which generalizes the more popular strategies in a threefold manner: We admit infinitely many degrees of freedom (by using infinite dimensional test function spaces $E$ ), we formulate the Weyl relations in terms of a possibly degenerate pre-symplectic form $\sigma$, and we start out for quantization from rather large classical Poisson algebras. An appropriate choice of the latter is basic for a rigorous quantization scheme, for which we join the ideas of the so-called strict deformation quantization. Because of the mentioned generalizations, we have however need for some technical pecularities, some of which we want to motivate in the finite dimensional case.

\subsection{Integration extension in finite dimensions}

Let us outline our approach to Weyl quantization at hand of the test function space $E:=\mathbb{R}^{d} \times \mathbb{R}^{d}$, $d \in \mathbb{N}$, which contains the coordinate tuples $f=(u, v)$ with $u, v \in \mathbb{R}^{d}$. The symplectic form $\sigma$

${ }^{\star}$ This paper is a contribution to the Special Issue on Deformation Quantization. The full collection is available at http://www.emis.de/journals/SIGMA/Deformation_Quantization.html 
is given by

$$
\sigma\left((u, v),\left(u^{\prime}, v^{\prime}\right)\right):=u \cdot v^{\prime}-v \cdot u^{\prime}, \quad \forall u, v, u^{\prime}, v^{\prime} \in \mathbb{R}^{d} .
$$

The dual space $E^{\prime}=\mathbb{R}^{d} \times \mathbb{R}^{d}$ serves as phase space for a system with $d$ degrees of freedom. The duality relation

$$
F(f)=u \cdot q+v \cdot p, \quad \forall F=[q, p] \in E^{\prime}, \quad \forall f=(u, v) \in E,
$$

imitates the smearing of the field in finite dimensions. (For smeared fields cf. e.g. [1, 2, 3]). A classical Weyl element, a "Weyl function", is given by the periodic phase space function

$$
W_{c}(f): E^{\prime} \longrightarrow \mathbb{C}, \quad F=[q, p] \longmapsto W_{c}(f)[F]=\exp \{i F(f)\}=\exp \{i(u \cdot q+v \cdot p)\},
$$

for each $f=(u, v) \in E$. According to Weyl [4, 5] one does not quantize the unbounded coordinate functions but the Weyl functions and uses the Schrödinger representation. So we do not adress here the question of further representations of the canonical commutation relations (CCR), inequivalent to the Schrödinger representation (e.g. $[6,7,8]$ ). We only mention von Neumann's uniqueness result [9] that the Schrödinger realization of the CCR is the unique (up to unitary equivalence) irreducible representation, for which the CCR arise from the Weyl relations.

Denoting in $\mathrm{L}^{2}\left(\mathbb{R}^{d}\right)$ the quantized momenta by $P_{k}=-i \hbar \frac{\partial}{\partial x_{k}}$ (depending on $\hbar \neq 0$ ) and the position operators (multiplication by the coordinate functions $x_{l}$ ) by $Q_{l}$, one arrives at the Weyl operators in the Schrödinger representation

$$
W_{S}^{\hbar}(u, v):=\exp \{i(u \cdot Q+v \cdot P)\}=\exp \left\{\frac{i}{2} \hbar u \cdot v\right\} \exp \{i u \cdot Q\} \exp \{i v \cdot P\}
$$

with $(u, v)=f \in E$. These unitary operators satisfy the Weyl relations

$$
W_{S}^{\hbar}(f) W_{S}^{\hbar}(g)=\exp \left\{-\frac{i}{2} \hbar \sigma(f, g)\right\} W_{S}^{\hbar}(f+g), \quad W_{S}^{\hbar}(f)^{*}=W_{S}^{\hbar}(-f), \quad \forall f, g \in E,
$$

and act as $\left(W_{S}^{\hbar}(u, v) \psi\right)(x)=\exp \left\{\frac{i}{2} \hbar u \cdot v\right\} \exp \{i u \cdot x\} \psi(x+\hbar v), \forall x \in \mathbb{R}^{d}$, on $\psi \in \mathrm{L}^{2}\left(\mathbb{R}^{d}\right)$. The smallest $C^{*}$-algebra containing all Weyl operators $W_{S}^{\hbar}(f), f \in E$, is $*$-isomorphic to the abstract $C^{*}$-Weyl algebra $\mathcal{W}(E, \hbar \sigma)$ over the symplectic space $(E, \hbar \sigma)$.

The concept of Weyl quantization includes a special operator ordering, implicitely given by the linear, *-preserving quantization map

$$
Q_{\hbar}^{S}\left(\sum_{k=1}^{n} z_{k} W_{c}\left(f_{k}\right)\right):=\sum_{k=1}^{n} z_{k} W_{S}^{\hbar}\left(f_{k}\right),
$$

with $n \in \mathbb{N}, z_{k} \in \mathbb{C}$, and with different $f_{k} \in E$.

The $C^{*}$-algebraic completion of this Weyl quantization covers also certain infinite sums, since $\sum_{k=1}^{\infty}\left|z_{k}\right|<\infty$ implies the convergence of $\sum_{k=1}^{\infty} z_{k} W_{c}\left(f_{k}\right)$ and $\sum_{k=1}^{\infty} z_{k} W_{S}^{\hbar}\left(f_{k}\right)$ in the $C^{*}$-norms (classically the supremum norm, and quantum mechanically the operator norm). (There exist of course $C^{*}$-algebraic quantization prescriptions different from the indicated symmetric Weyl ordering cf. [10, 11], and references therein.)

Because $E \ni f \mapsto W_{S}^{\hbar}(f)$ is discontinuous with respect to the operator norm, an extension of the quantization map $Q_{\hbar}^{S}$ to more general functions than the almost periodic functions of the type $\sum_{k=1}^{\infty} z_{k} W_{c}\left(f_{k}\right)$ is impossible in terms of the norm. However, we are dealing with a representation, the Schrödinger representation, of the abstract Weyl algebra $\mathcal{W}(E, \hbar \sigma)$, and so there exist weaker topologies than the norm. And indeed, $E \ni f \mapsto W_{S}^{\hbar}(f)$ is continuous with respect to all weak 
operator topologies (weak, $\sigma$-weak, strong, ...). This allows for extending the quantization map $Q_{\hbar}^{S}$ to suitable "continuous linear combinations" of the periodic functions $W_{c}(f)$ : Let $\mathcal{M}(E)$ be the finite, complex, regular Borel measures on $E$. The Fourier transform $\widehat{\mu}[F]:=$ $\int_{E} d \mu(f) \exp \{i F(f)\}, F \in E^{\prime}$, of the measure $\mu \in \mathcal{M}(E)$ is a bounded, continuous function $\widehat{\mu}: E^{\prime} \rightarrow \mathbb{C}$, which may be considered as the point-wise "continuous linear combination"

$$
\widehat{\mu}=\int_{E} d \mu(f) W_{c}(f)
$$

of the $W_{c}(f), f \in E$. So the desired extension of $Q_{\hbar}^{S}$ from the original linear combinations in equation (1.3) to the "continuous" ones from (1.4) is given by

$$
Q_{\hbar}^{S}(\widehat{\mu}):=\int_{E} d \mu(f) Q_{\hbar}^{S}\left(W_{c}(f)\right)=\int_{E} d \mu(f) W_{S}^{\hbar}(f) .
$$

The integral exists - as the limit of the Lebesgue partial sums - with respect to the weak operator topologies, constituting a bounded operator in $\mathrm{L}^{2}\left(\mathbb{R}^{d}\right)$. Especially, the above infinite series

$$
Q_{\hbar}^{S}(\widehat{\mu})=Q_{\hbar}^{S}\left(\sum_{k=1}^{\infty} z_{k} W_{c}\left(f_{k}\right)\right)=\sum_{k=1}^{\infty} z_{k} W_{S}^{\hbar}\left(f_{k}\right)
$$

just belong to the discrete measures $\mu=\sum_{k=1}^{\infty} z_{k} \delta\left(f_{k}\right) \in \mathcal{M}(E)_{d}$ on $E$ with finite total weights $\sum_{k=1}^{\infty}\left|z_{k}\right|<\infty$ (where $\delta(f)$ denotes the point measure at $f \in E$ ). Therefore, the map in (1.5) provides, already for finite dimensions, a considerable extension of the $C^{*}$-algebraic Weyl quantization by means of weak integration in a representation space. This extension is comparable to the transition from Fourier series to Fourier integrals, where the Fourier transformations of finite measures are continuous bounded functions. (Certain classes are described at the end of the paper.)

The connection to deformation quantization is gained by observing that $Q_{\hbar}^{S}$ acts injectively on the Fourier transformed measures. Hence one may define a non-commutative product for a certain class of phase space functions by setting

$$
\widehat{\mu} \cdot \hbar:=Q_{\hbar}^{S^{-1}}\left(Q_{\hbar}^{S}(\widehat{\mu}) Q_{\hbar}^{S}(\widehat{\nu})\right),
$$

where $Q_{\hbar}^{S}(\widehat{\mu}) Q_{\hbar}^{S}(\widehat{\nu})$ means the common operator product.

The essence of quantization may be considered as deforming the commutative, pointwise product for phase space functions into a non-commutative, $\hbar$-dependent product like $\widehat{\mu} \hbar \widehat{\nu}$. (Explicit formulations are given in the form of so-called Moyal products.) This point of view has certainly provoked many fresh ideas on the quantization problem. In this connection let us add as a side remark that, keeping the phase space functions as observables also in the quantized theory, speaks a bit against the philosophy that the canonical observables may not "exist" before being measured (and supports perhaps Einstein's side in the Einstein-Bohr debate).

In spite of the merits of the quantized phase space formalism we concentrate in the present investigation first on "quantizing" measures on the test function space. Before Fourier transformation, the deformed product (1.7) is just the twisted convolution

$$
\mu \star \hbar \nu=\mathbb{F}^{-1}(\widehat{\mu} \cdot \hbar \widehat{\nu}), \quad \forall \mu, \nu \in \mathcal{M}(E),
$$

on the measure space $\mathcal{M}(E)$ with respect to the multiplier $\exp \left\{-\frac{i}{2} \hbar \sigma(f, g)\right\}$ occurring in the Weyl relations (1.2). (As forerunners may be mentioned [12] and even [9].) 
Equipped with a suitable involution and the twisted convolution product, $\left(\mathcal{M}(E), \star_{\hbar}\right)$ constitutes a Banach-*-algebra with respect to the total variation norm. This leads us to a different reading of formula (1.5): On the right hand side we see the Weyl operators $W_{S}^{\hbar}(f)$ in the Schrödinger representation, which are integrated over the measure $\mu$ to an operator, which in general is no longer contained in the represented $C^{*}$-Weyl algebra. For varying $\mu \in \mathcal{M}(E)$ the resulting operators realize $*$-homomorphically the $*$-algebraic operations of $\left(\mathcal{M}(E), \star_{\hbar}\right)$ in terms of the hermitian conjugation and operator product. Thus the mapping $\mu \mapsto Q_{\hbar}^{S}(\widehat{\mu})$ provides us with a so-called regular representation of the Banach-*-algebra $\left(\mathcal{M}(E), \star_{\hbar}\right)$.

For such structures there exist much mathematical literature related to locally compact groups, to which we have to refer frequently. Still another interpretation of the quantization formula (1.5) results from this field of mathematics. In virtue of the Weyl relations (1.2), the mapping $E \ni f \mapsto W_{S}^{\hbar}(f)$ realizes a $\sigma$-strongly continuous projective unitary representation of the locally compact vector group $E$. Integration over the group leads to the realm of twisted group algebras.

In any case, we emphasize the use of the Banach-*-algebras $\left(\mathcal{M}(E), \star_{\hbar}\right)$. We do this in modified form also in the infinite dimensional case, as is sketched in the next Subsection. Since the extension of the (represented) $C^{*}$-Weyl algebra is performed by weak integration we term it integration extension. Related with this are the integration type representations of $\left(\mathcal{M}(E)\right.$, $\left.\star_{\hbar}\right)$.

\subsection{Overview on the extension of field theoretic Weyl quantization}

Extending of the ideas of the previous Subsection to infinite dimensional test function spaces $E$, where already usual $C^{*}$-algebraic Weyl quantization over such $E$ offers certain subtleties (e.g. $[13,14]$ ), causes additional mathematical difficulties, the treatment of which forms the subject of the present investigation. Let us at this place give only some motivation and an overview on the main steps of reasoning.

Concerning the generalization of the symplectic form, there are, in fact, many instances of field theories with pre-symplectic test function spaces. In the formulation of quantum electrodynamics (QED) for non-relativistic optical systems the transversal parts of the canonical field variables have to be separated out by means of a Helmholtz-Hodge decomposition (cf. e.g. $[15,16,17])$. Only these enter the canonical formalism (in an infinite cavity) and are quantized. The corresponding Poisson tensor for the total field in the Coulomb gauge is thus highly degenerate. In field theory for high energy physics, there are degenerate symplectic forms, for example, in the frame of conformal field theory (cf. e.g. [18]). Quite generally, field theories with intrinsic superselection rules require CCR resulting from degenerate Poisson brackets.

Also in the frame of deformation quantization, stipulated by the seminal work of [19], diverse attempts were undertaken to meet the challenges of quantum field theory (QFT) (cf. [20, 21, $22,23,24])$. The various so-called star exponentials cover a wide range of bounded functions on the field phase space. Different kinds of ordering for unbounded field polynomials occur in their series expansions. As pointed out especially by Dito [25, 20] the requirement that the star exponential of the Hamiltonian be well defined restricts the class of suitable deformed products (star products) to some extent, leaving still open a wide class of possible deformations. Most of these formulations have not reached full mathematical rigor, but they give inspiration to extend the controlled formalism as much as possible and to systematize the deformations of field expressions.

Our mentioned previous works on Weyl quantization over infinitely many degrees of freedom $[14,11]$ were based on a more restrictive notion of deformation quantization, termed strict by Rieffel and Landsman [26, 10, 27, 28, 29], and outlined briefly in Subsection 2.1. Our mathematical techniques avoid, however, phase space integrals of the quantized products and commutators, basic for the finite dimensional deformation quantization (also used in the papers 
of Rieffel and Landsman), since these are hard to generalize to infinitely many variables.

For definiteness consider the abstract Weyl relations

$$
W^{\hbar}(f) W^{\hbar}(g)=\exp \left\{-\frac{i}{2} \hbar \sigma(f, g)\right\} W^{\hbar}(f+g), \quad W^{\hbar}(f)^{*}=W^{\hbar}(-f), \quad \forall f, g \in E,
$$

where $E$ denotes the infinite dimensional test function space, equipped with the (real bilinear, antisymmetric) pre-symplectic form $\sigma$. According to the deformation strategy, $\hbar$ is a variable parameter, which we let range in $\mathbb{R}$. Generally we assume $\sigma \neq 0$, implying (partial) noncommutativity in the Weyl relations (1.8) for the quantum regime $\hbar \neq 0$.

For each $\hbar \in \mathbb{R}$ the (finite) linear combinations of Weyl elements $W^{\hbar}(f), f \in E$, determine already the abstract $C^{*}$-Weyl algebra $\mathcal{W}(E, \hbar \sigma)$, also in the case of degenerate $\sigma$, cf. Subsection 2.2. This uniqueness is achieved by demanding the abstract $C^{*}$-Weyl algebra to include all intrinsic superselection observables. For mutually different values of $\hbar$ the associated Weyl relations lead to different Weyl algebras $\mathcal{W}(E, \hbar \sigma)$, possessing different norms. In Subsection 2.3 we investigate transformations between the $\mathcal{W}(E, \hbar \sigma)$ with different $\hbar$. As is recapitulated in Subsection 2.4, the $C^{*}$-norms are so well calibrated that the Weyl quantization, induced by the mappings

$$
Q_{\hbar}\left(\sum_{k=1}^{\infty} z_{k} W_{c}\left(f_{k}\right)\right):=\sum_{k=1}^{\infty} z_{k} W^{\hbar}\left(f_{k}\right) \in \mathcal{W}(E, \hbar \sigma),
$$

satisfies all axioms of a strict deformation quantization, including Rieffel's condition. The geometric features behind the choice of the classical Poisson bracket are touched upon in Subsection 2.5. As mentioned for finite dimensions in Subsection 1.1, the dual space $E_{\tau}^{\prime}$, formed with respect to some locally convex (vector space) topology $\tau$, serves as the (flat) phase space manifold of the classical field theory. The classical Weyl elements $W^{0}(f), f \in E$, may be realized by the continuous, periodic functions $W_{c}(f)[F]:=\exp \{i F(f)\}$ on $E_{\tau}^{\prime} \ni F$. We emphasize however the algebraic universality of this basic mechanical structure and of the whole quantization method, involving the $\mathcal{W}(E, \hbar \sigma)$, which expresses a functorial dependence on $(E, \sigma)$.

The first step for the extended theory in Section 3 is the introduction of the appropriate, universal measure space $\mathcal{M}(E)$ in terms of an inductive limit. The Banach-*-algebras of measures $\left(\mathcal{M}(E), \star_{\hbar}\right)$, with the (deformed) convolution products (equation (3.4) below) and endowed with the total variation norm, for each $\hbar \in \mathbb{R}$, use all of $\mathcal{M}(E)$. Certain semi-norms compatible with $\sigma$ are introduced for constructing the Poisson algebras of measures. The existence of their moments with respect to a measure imitates differentiability assumptions on the phase space function obtained from the measure by Fourier transformation.

Let us mention that in [13] D. Kastler already introduced $(\mathcal{M}(E), \star \hbar)$ for the construction of a Boson field $C^{*}$-algebra in terms of a $C^{*}$-norm completion, which depends on a Schrödinger-like representation. There this construction has been used, however, for non-degenerate $\sigma$ (and fixed $\hbar \neq 0)$, only.

For proving the axioms of strict deformation quantization in the Banach-*-type version, the $\hbar$-independence of the variation norm is a great help.

Section 4 treats the Poisson algebras in the more common phase space formulation. Here, but also in the previous measure version, the observables of the classical and of the quantized theory are the same mathematical quantities. Our remarks in Subsection 4.2 on the pre-symplectic geometry give a glimpse on the difficulties one has in dealing with Hamiltonian vector fields in infinite dimensions. The introduction of restricted tangent and cotangent spaces touches the problem of the foliation into symplectic leaves. Our contribution is the smooth adjunction of such geometrical structures to the quantized field theory.

From the finite dimensional theory one knows how useful a rigorous classical approximation to the quantum theory is, both for technical and interpretational reasons. 
The step to the $C^{*}$-version of strict field quantization requires additional mathematical tools. In Section 5 certain representations of the $\left(\mathcal{M}(E), \star_{\hbar}\right)$, termed integration type representations, are investigated. They have the form

$$
\Pi_{\hbar}(\mu)=\int_{E} d \mu(f) \pi_{\hbar}(f), \quad \forall \mu \in \mathcal{M}(E),
$$

where $E \ni f \mapsto \pi_{\hbar}(f)$ is a $\sigma$-strongly continuous, projective, unitary representation of the vector group $E$ arising from the Weyl relations. The connection to a representation of the original $C^{*}$-Weyl algebra $\mathcal{W}(E, \hbar \sigma)$, resp. of $\left(\mathcal{M}(E)_{d}, \star \hbar\right)$, is given by

$$
\pi_{\hbar}(f)=\Pi_{\hbar}\left(W^{\hbar}(f)\right)=\Pi_{\hbar}(\delta(f)), \quad \forall f \in E,
$$

and the representation $\Pi_{\hbar}$ extends from $\left(\mathcal{M}(E)_{d}, \star_{\hbar}\right)$ to all measures $\mathcal{M}(E)$ via (1.9).

The consideration of representations is necessary, because the $C^{*}$-norm of the enveloping $C^{*}$-algebra is gained by a supremum over the representation norms (cf. Subsection 2.1). Regularity of a representation of $\mathcal{W}(E, \hbar \sigma)$, which allows for the representation dependent Boson field operators by differentiating the represented Weyl elements, has to be strengthened to our concept of $\tau$-continuity. The normal states of the $\tau$-continuous representations constitute the $\tau$-continuous folia. They play, quite generally, an important role for Boson field theory e.g. for formulating the dynamics and for a spatial decomposition theory of the non-separable $C^{*}$-Weyl algebra $\mathcal{W}(E, \hbar \sigma)$. Since they are not so well known we treat them in some detail. In the present context they are inserted into the integration type extensions (1.9) of representations (which display not all of the desired properties if only regular representations are inserted).

In Section 6 we finally deal with the extended Weyl quantization in the $C^{*}$-version. The quantization maps are based on the integration representations

$$
Q_{\hbar}^{\Pi}(\widehat{\mu}):=\Pi_{\hbar}(\mu), \quad \forall \mu \in \mathcal{M}(E) .
$$

The need of an intermediate step via Hilbert space representations causes great difficulties when using a degenerate symplectic form $\sigma$. Now the intrinsic superselection observables may be partially lost in a representation, causing deficits in the $C^{*}$-norms. To calibrate these losses we work with so-called well-matched families of ( $\tau$-continuous and regular) representations (indexed by $\hbar$ ). Under these assumptions, partial results on the Rieffel condition are derived, whereas the even more popular Dirac and von Neumann conditions provide no difficulties.

Altogether, the classical correspondence limit is concisely worked out for a rather comprehensive set of observables. Subclasses of these are described at the end of Section 6 in terms of phase space functions. The other way round, our investigation illustrates that deformation quantization in infinite dimensions requires adequate topological and measure theoretic notions. Some are connected here with Hilbert space representations, which may however be replaced by the choice of state folia, since the latter serve equally well to define weak topologies (and integration and differentiation methods) in the observable algebras.

Physically the choice of a folium expresses classical, macroscopic, i.e. collective, aspects of the quantum field system. Disjoint folia describe different features of the macroscopic preparation of the system (as e.g. different reservoir couplings, like heat baths or weak links to condensed particles). They lead to additional superselection sectors (represented also by additional central observables in the weak closures) to the intrinsic ones, originating from the algebraic degeneracy of the commutation relations. (Charges are intrinsic, thermodynamic variables parametrize folia.)

Due to the described extension of the algebraic structure in comparison to the usual $C^{*}$ Weyl algebra of Boson fields, one may profit now from a still wider class of field operator realizations than in traditional algebraic QFT. This may be of use for improving the more 
heuristic approaches to infinite dimensional deformation quantization, which employ directly the field observables (in contradistinction to the bounded Weyl elements). Since the unbounded field operators depend essentially on the representation, resp. on the chosen weak topology (e.g. in a GNS representation over a condensed state arise additional classical field parts), the classification of their deformed products has to take into account, beside - and in combination with - operator ordering, the effects of weak topologies.

\section{Preliminary notions and results}

Let us first make some notational remarks. Generally all occurring topologies are assumed to be Hausdorff. If not specified otherwise (bi-)linearity is understood over the complex field $\mathbb{C}$. The linear hull $\operatorname{LH}\{V\}$ denotes the (finite) complex linear combinations of the elements of the set $V$.

We deal exclusively with $*$-algebras $\mathcal{A}$ (containing the arbitrary elements $A$ and $B$ ) over the complex field $\mathbb{C}$ with an associative, but possibly non-commutative product. The $*$-operation is involutive $\left(\left(A^{*}\right)^{*}=A\right)$, anti-linear, and product anti-homomorphic $\left((A B)^{*}=B^{*} A^{*}\right)$. An algebra norm is a vector space norm with $\|A B\| \leq\|A\|\|B\|$. A Banach algebra is an algebra, which is complete in an algebra norm. A Banach-*-algebra is a Banach algebra with a *operation, which satisfies $\left\|A^{*}\right\|=\|A\|$. A $C^{*}$-norm $\|\cdot\|$ on a $*$-algebra $\mathcal{A}$ is an algebra norm satisfying the so-called $C^{*}$-norm property $\left\|A^{*} A\right\|=\|A\|^{2}$. A $C^{*}$-algebra is a Banach-*-algebra the norm of which is a $C^{*}$-norm.

Under a representation $\left(\Pi, \mathcal{H}_{\Pi}\right)$ of a $*$-algebra $\mathcal{A}$ we understand a $*$-homomorphism $\Pi$ from $\mathcal{A}$ into the $C^{*}$-algebra of all bounded operators on the complex representation Hilbert space $\mathcal{H}_{\Pi}$. If $\left(\Pi, \mathcal{H}_{\Pi}\right)$ represents a Banach-*-algebra, then $\|\Pi(A)\| \leq\|A\|$. If $\left(\Pi, \mathcal{H}_{\Pi}\right)$ is a faithful representation of a $C^{*}$-algebra, then $\|\Pi(A)\|=\|A\|$. If $\mathcal{A}$ is a Banach-*-algebra one introduces a $C^{*}$-norm by setting $\|A\|_{C^{*}}:=\sup \{\|\Pi(A)\|\}$, where the supremum goes over all representations. The enveloping $C^{*}$-algebra $\mathcal{C}(\mathcal{A})$ of a Banach-*-algebra $\mathcal{A}$ is the completion of $\mathcal{A}$ in the mentioned $C^{*}$-norm.

The commutative $C^{*}$-algebra $\left(\mathrm{C}_{b}(\mathrm{P}), 0\right)$, consisting of the bounded, continuous functions $A: \mathrm{P} \rightarrow \mathbb{C}, F \mapsto A[F]$ on the topological space $\mathrm{P}$ has the sup-norm $\|\cdot\|_{0}$ as its $C^{*}$-norm, $\|A\|_{0}=\sup \{|A[F]| \mid F \in \mathrm{P}\}$, and is equipped with the usual pointwise-defined commutative *-algebraic operations

$$
\left(A_{0} B\right)[F]:=A[F] B[F], \quad A^{*}[F]:=\overline{A[F]}, \quad \forall F \in \mathrm{P} .
$$

\subsection{The notion of strict deformation quantization}

Bohr's correspondence principle has reached an especially concise form in terms of strict deformation quantization. Let us specify this notion.

A Poisson algebra $(\mathcal{P},\{\cdot, \cdot\})$ consists of a commutative $*$-algebra $\left(\mathcal{P},{ }_{0}\right)$ (over $\mathbb{C}$ by the above notational remarks) equipped with a Poisson bracket $\{\cdot, \cdot\}$. The latter is a bilinear mapping $\{\cdot, \cdot\}: \mathcal{P} \times \mathcal{P} \rightarrow \mathcal{P}$, which is anticommutative $\{A, B\}=-\{B, A\}$, real $\{A, B\}^{*}=\left\{A^{*}, B^{*}\right\}$, fulfills the Jacobi identity $\{A,\{B, C\}\}+\{B,\{C, A\}\}+\{C,\{A, B\}\}=0$, and the Leibniz rule $\left\{A, B{ }_{0} C\right\}=\{A, B\}{ }_{0} C+B{ }_{0}\{A, C\} . \mathcal{P}$ is assumed to be $\|\cdot\|_{0}$-dense in a commutative $C^{*}$-algebra $\mathcal{A}^{0}\left(\|\cdot\|_{0}\right.$ denotes the $C^{*}$-norm on $\left.\mathcal{A}^{0}\right)$, where $\mathcal{A}^{0}$ is interpreted as the algebra of observables for the considered classical field theory.

In so-called "algebraic quantum field theory" a quantum field system is characterized in terms of a non-commutative $C^{*}$-algebra $\mathcal{A}^{\hbar}$, equipped with the $C^{*}$-norm $\|\cdot\|_{\hbar}$, on which the $\hbar$-scaled commutator be defined by

$$
\{A, B\}_{\hbar}:=\frac{i}{\hbar}(A B-B A), \quad \forall A, B \in \mathcal{A}^{\hbar} .
$$


The quantum system should be defined for so many values of $\hbar \neq 0$ that the classical correspondence limit $\hbar \rightarrow 0$ may be investigated. A subset $J \subseteq \mathbb{R}$, containing 0 as an accumulation point of $J_{0}:=J \backslash\{0\}$ would be sufficient. But, for simplicity, in the present work we choose mostly $J=\mathbb{R}$.

For each $\hbar \in J_{0}$ let us be given a quantization map $Q_{\hbar}: \mathcal{P} \rightarrow \mathcal{A}^{\hbar}$, which tells how the quantum observables are related to given classical ones. $Q_{\hbar}$ is supposed linear and $*$-preserving, that is $Q_{\hbar}\left(A^{*}\right)=Q_{\hbar}(A)^{*}$ for all $A \in \mathcal{P}$. Clearly, $Q_{\hbar}$ cannot respect the commutative products of $\mathcal{P}$.

Definition 1 (Strict deformation quantization). Let $J \subseteq \mathbb{R}$ be as specified above. A strict quantization $\left(Q_{\hbar}\right)_{\hbar \in J}$ of the Poisson algebra $(\mathcal{P},\{\cdot, \cdot\})$ consists for each value $\hbar \in J$ of a linear, *-preserving map $Q_{\hbar}: \mathcal{P} \rightarrow \mathcal{A}^{\hbar}$ ( $\mathcal{A}^{\hbar}$ being a $C^{*}$-algebra), such that $Q_{0}$ is the identical embedding and such that for all $A, B \in \mathcal{P}$ the following conditions are fulfilled:

(a) [Dirac's condition] The $\hbar$-scaled commutator (2.2) approaches the Poisson bracket as $0 \neq \hbar \rightarrow 0$, that is, $\lim _{\hbar \rightarrow 0}\left\|\left\{Q_{\hbar}(A), Q_{\hbar}(B)\right\}_{\hbar}-Q_{\hbar}(\{A, B\})\right\|_{\hbar}=0$.

(b) [von Neumann's condition] As $\hbar \rightarrow 0$ one has the asymptotic homomorphism property $\lim _{\hbar \rightarrow 0}\left\|Q_{\hbar}(A) Q_{\hbar}(B)-Q_{\hbar}\left(A_{0} B\right)\right\|_{\hbar}=0$.

(c) [Rieffel's condition] $J \ni \hbar \mapsto\left\|Q_{\hbar}(A)\right\|_{\hbar}$ is continuous.

The strict quantization $\left(Q_{\hbar}\right)_{\hbar \in J}$ is called a strict deformation quantization, if each $Q_{\hbar}$ is injective and if its image $Q_{\hbar}(\mathcal{P})$ is a sub-*-algebra of $\mathcal{A}^{\hbar}$.

For a strict deformation quantization one may define on $\mathcal{P}$ the deformed product

$$
A_{\hbar} B:=Q_{\hbar}^{-1}\left(Q_{\hbar}(A) Q_{\hbar}(B)\right), \quad \forall A, B \in \mathcal{P},
$$

ensuring $(\mathcal{P}, \hbar)$ to be a non-commutative $*$-algebra, $*$-isomorphic to $Q_{\hbar}(\mathcal{P})$.

In Subsection 3.4 we deal with a Banach-*-algebra version of strict deformation quantization, where the above $C^{*}$-algebras $\mathcal{A}^{\hbar}$ are replaced by Banach-*-algebras. We distinguish the two notions of a strict deformation quantization by calling them to be of $C^{*}$-type resp. of Banach*-type.

\subsection{Weyl algebra}

Let us have a pre-symplectic space $(E, \sigma)$. Recall by the way that $\sigma$ is symplectic, if its null space

$$
\operatorname{ker}_{\sigma}:=\{f \in E \mid \sigma(f, g)=0 \forall g \in E\}
$$

is trivial. The standard example of a symplectic space $E$ is a complex pre-Hilbert space, regarded as a real vector space, where $\sigma$ is the imaginary part of its complex inner product $(\cdot \mid \cdot)$.

Let us fix an arbitrary value $\hbar \in \mathbb{R}$. For $E$, considered as a topological vector group with respect to the discrete topology, we define the multiplier

$$
E \times E \ni(f, g) \longmapsto \exp \left\{-\frac{i}{2} \hbar \sigma(f, g)\right\} .
$$

In order to construct for $E$ the twisted group Banach-*- resp. $C^{*}$-algebra we start from an abstract $*$-algebra, given as the formal linear hull

$$
\Delta(E, \hbar \sigma):=\operatorname{LH}\left\{W^{\hbar}(f) \mid f \in E\right\}
$$


of linearly independent symbols $W^{\hbar}(f), f \in E$, called Weyl elements. Equipped with the Weyl relations

$$
W^{\hbar}(f) W^{\hbar}(g)=\exp \left\{-\frac{i}{2} \hbar \sigma(f, g)\right\} W^{\hbar}(f+g), \quad W^{\hbar}(f)^{*}=W^{\hbar}(-f), \quad \forall f, g \in E,
$$

$\Delta(E, \hbar \sigma)$ indeed becomes a $*$-algebra. Its identity is given by $\mathbb{1}^{\hbar}:=W^{\hbar}(0)$, and every Weyl element $W^{\hbar}(f)$ is unitary $\left(W^{\hbar}(f)^{*}=W^{\hbar}(f)^{-1}\right)$.

The completion $\overline{\Delta(E, \hbar \sigma)}^{1}$ with respect to the norm

$$
\left\|\sum_{k=1}^{n} z_{k} W^{\hbar}\left(f_{k}\right)\right\|_{1}:=\sum_{k=1}^{n}\left|z_{k}\right|, \quad n \in \mathbb{N}, z_{k} \in \mathbb{C}, \text { different } f_{k} \text { 's from } E,
$$

is just the twisted group Banach-*-algebra of $E$. The Weyl algebra $\mathcal{W}(E, \hbar \sigma)$ over the presymplectic space is by definition the twisted group $C^{*}$-algebra of $E$, i.e., the enveloping $C^{*}$ algebra of the Banach-*-algebra $\overline{\Delta(E, \hbar \sigma)}^{1}$. (For twisted group algebras we defer the reader to the citations in Subsection 3.2, and for the Weyl algebra with degenerate $\sigma$ see [30,31], and references therein.) The $C^{*}$-norm on $\mathcal{W}(E, \hbar \sigma)$ is denoted by $\|\cdot\|_{\hbar}$. It varies with different values of $\hbar \in \mathbb{R}$, in contrast to the $\hbar$-independent Banach norm $\|\cdot\|_{1} \cdot \overline{\Delta(E, \hbar \sigma)}^{1}$ is a proper, but $\|\cdot\|_{\hbar}$-dense sub-*-algebra of $\mathcal{W}(E, \hbar \sigma)$, where (see the definitions at the beginning of this Section)

$$
\|A\|_{\hbar} \leq\|A\|_{1}, \quad \forall A \in \overline{\Delta(E, \hbar \sigma)}^{1} .
$$

By construction of the enveloping $C^{*}$-algebra, the states and representations of $\mathcal{W}(E, \hbar \sigma)$ and $\overline{\Delta(E, \hbar \sigma)}^{1}$ are in 1:1-correspondence, given by continuous extension resp. restriction. The $C^{*}$ Weyl algebra $\mathcal{W}(E, \hbar \sigma)$ is simple, if and only if $\sigma$ is non-degenerate and $\hbar \neq 0$.

If $\pi_{\hbar}$ is any projective unitary representation of the additive group $E$ with respect to the multiplier (2.5), then there exists a unique non-degenerate representation $\Pi_{\hbar}$ of $\mathcal{W}(E, \hbar \sigma)$ (and by restriction of $\left.\overline{\Delta(E, \hbar \sigma)}^{1}\right)$ such that

$$
\pi_{\hbar}(f)=\Pi_{\hbar}\left(W^{\hbar}(f)\right), \quad \forall f \in E,
$$

and conversely. Whenever we use in the sequel both notions $\pi_{\hbar}$ and $\Pi_{\hbar}$, they are connected as described here. For $f \neq g$ we have $\left\|W^{\hbar}(f)-W^{\hbar}(g)\right\|_{\hbar}=2$, which implies discontinuity of $f \mapsto W^{\hbar}(f)$ in the norm. But for certain projective group representations $\pi_{\hbar}$, the mapping $E \ni f \mapsto \pi_{\hbar}(f)$ may be strongly continuous with respect to some topology $\tau$ on $E$. This point is essential for the present investigation and considered in more detail in Subsection 5.2 below.

\section{$2.3 *$-isomorphisms for the quantum Weyl algebras}

For $\hbar=0$ the multiplier (2.5) becomes trivial, which leads to the commutative $C^{*}$-Weyl algebra $\mathcal{W}(E, 0)$. For the quantum cases $\hbar \neq 0$ all Weyl algebras $\mathcal{W}(E, \hbar \sigma)$ are $*$-isomorphic, a fact which we need in Section 6 to make representations $\Pi_{\hbar}$ of $\mathcal{W}(E, \hbar \sigma)$, with different $\hbar$, compatible with each other.

Lemma 1. For $\hbar \neq 0$ let $T_{\hbar}: E \rightarrow E$ be an $\mathbb{R}$-linear bijection such that $\sigma\left(T_{\hbar} f, T_{\hbar} g\right)=\hbar \sigma(f, g)$ for all $f, g \in E$. Then there exists a unique *isomorphism $\beta_{\hbar}$ from $\mathcal{W}(E, \hbar \sigma)$ onto $\mathcal{W}(E, \sigma)$ (where $\hbar=1$ ) such that $\beta_{\hbar}\left(W^{\hbar}(f)\right)=W^{1}\left(T_{\hbar} f\right)$ for all $f \in E$.

If in addition $T_{\hbar}$ is a homeomorphism with respect to $\tau$, then the dual mapping $\beta_{\hbar}^{*}$ is an affine bijection from $\mathcal{F}_{\hbar=1}^{\tau}$ onto $\mathcal{F}_{\hbar}^{\tau}$.

(The convex folia $\mathcal{F}_{\hbar}^{\tau}$ of $\tau$-continuous states are introduced in Subsection 5.2.) 
Proof. In virtue of the linear independence of the $W^{\hbar}(f), f \in E, \beta_{\hbar}$ is a well-defined *isomorphism from $\Delta(E, \hbar \sigma)$ onto $\Delta(E, \sigma)$. Extend norm-continuously using a result in [31].

A first idea to construct a $T_{\hbar}$ would be

$$
T_{\hbar} f:=\sqrt{|\hbar|} f, \quad \forall f \in E .
$$

But here it follows for $\hbar<0$ that $\beta_{\hbar}$ is a $*$-anti-isomorphism (antilinear $*$-isomorphism), and thus either the initial or the final folium has to consist of antilinear states.

Let us try a second example. Suppose the existence of a conjugation $C$ on $(E, \sigma)$, that is, $C: E \rightarrow E$ is an $\mathbb{R}$-linear mapping satisfying $C^{2} f=f$ and $\sigma(C f, g)=-\sigma(f, C g)$ for all $f, g \in E$. The eigenspaces $E_{+}$and $E_{-}$of $C$ are given by $E_{ \pm}:=\{f \in E \mid C f= \pm f\}=\{g \pm C g \mid g \in E\}$, and the associated spectral projections $P_{ \pm}$by $P_{ \pm} g=\frac{1}{2}(g \pm C g)$ for $g \in E$. Clearly $E_{+} \bigcap E_{-}=\{0\}$ and every $f \in E$ decomposes uniquely as $f=P_{+} f+P_{-} f$ (in analogy to a polarization of a phase space).

One finds $\sigma\left(P_{+} f, P_{+} g\right)=0=\sigma\left(P_{-} f, P_{-} g\right)$, thus $\sigma(f, g)=\sigma\left(P_{+} f, P_{-} g\right)+\sigma\left(P_{-} f, P_{+} g\right)$ for all $f, g \in E$. For $\hbar \neq 0$ we define an $\mathbb{R}$-linear bijection

$$
T_{\hbar} f:=\theta_{+}(\hbar) P_{+} f+\theta_{-}(\hbar) P_{-} f, \quad \forall f \in E,
$$

where the real values $\theta_{+}(\hbar)$ and $\theta_{-}(\hbar)$ have to satisfy $\theta_{+}(\hbar) \theta_{-}(\hbar)=\hbar$. Then $\sigma\left(T_{\hbar} f, T_{\hbar} g\right)=$ $\hbar \sigma(f, g)$, and thus Lemma 1 is applicable. If $C$ is $\tau$-continuous, then $P_{ \pm}$are so, and thus it follows that $T_{\hbar}$ is a homeomorphism of $E$.

One may assume in addition a complex structure $j$ on $(E, \sigma)(j$ is an $\mathbb{R}$-linear mapping on $E$ satisfying $j^{2} f=-f, \sigma(j f, g)=-\sigma(f, j g)$, and $\sigma(f, j f) \geq 0$, for all $\left.f, g \in E\right)$, such that $C$ is just a complex conjugation for $j$, i.e., $C j=-j C$. Then $E$ becomes a complex vector space with the multiplication $z f=\Re(z) f+j \Im(z) f, f \in E, z \in \mathbb{C}$, carrying the associated complex semi-inner product $(f \mid g)_{j}:=\sigma(f, j g)+i \sigma(f, g)$, for $f, g \in E$. Since $E_{-}=j E_{+}$resp. $P_{-} j=j P_{+}$, we have $E=E_{+}+j E_{+}$, that is the complexification of $E_{+}$. Now, $T_{\hbar}(u+j v)=\theta_{+}(\hbar) u+j \theta_{-}(\hbar) v$ for all $u, v \in E_{+}$. Clearly, $C$ and $j$ are highly non-unique. A finite dimensional example, fitting to Subsection 1.1, is $T_{\hbar}(u+i v):=\hbar u+i v$ for all $u, v \in \mathbb{R}^{d}$.

\subsection{Strict deformation quantization for $C^{*}$-Weyl algebras}

For the classical case $\hbar=0$ it turns out that the abstract commutative $*$-algebra $\Delta(E, 0)$ becomes a Poisson algebra $\left(\Delta(E, 0),\{\cdot, \cdot\}_{0}\right)$, where the Poisson bracket $\{\cdot, \cdot\}_{0}$ is given by the bilinear extension of the algebraic relations

$$
\left\{W^{0}(f), W^{0}(g)\right\}_{0}=\sigma(f, g) W^{0}(f+g), \quad \forall f, g \in E .
$$

As indicated already in Section 1 for each $\hbar \in \mathbb{R}$ the Weyl quantization map $Q_{\hbar}: \Delta(E, 0) \rightarrow$ $\mathcal{W}(E, \hbar \sigma)$ is defined by the linear extension of

$$
Q_{\hbar}\left(W^{0}(f)\right):=W^{\hbar}(f), \quad \forall f \in E,
$$

(which is well defined since the Weyl elements $W^{\hbar}(f), f \in E$, are linearly independent for every $\hbar \in \mathbb{R})$. Obviously, $Q_{\hbar}$ is a linear, *-preserving $\|\cdot\|_{1}-\|\cdot\|_{1}$-isometry from $\Delta(E, 0)$ onto $\Delta(E, \hbar \sigma)$. Using the $C^{*}$-norms, one recognizes as an intermediate result in the course of the present investigation:

Theorem 1 ([14]). The family of mappings $\left(Q_{\hbar}\right)_{\hbar \in \mathbb{R}}$ constitutes a strict deformation quantization (of $C^{*}$-type) of the Poisson algebra $\left(\Delta(E, 0),\{\cdot, \cdot\}_{0}\right)$. 


\subsection{Geometry and algebraization of a classical field theory}

Suppose a locally convex topology $\tau$ on the real vector space $E$, the $\tau$-topological dual of which be denoted by $E_{\tau}^{\prime}$. On $E_{\tau}^{\prime}$ we choose the $\sigma\left(E_{\tau}^{\prime}, E\right)$-topology and denote by $\left(\mathrm{C}_{b}\left(E_{\tau}^{\prime}\right)\right.$, $\left.{ }_{0}\right)$ the commutative $C^{*}$-algebra of all bounded, $\sigma\left(E_{\tau}^{\prime}, E\right)$-continuous functions $A: E_{\tau} \rightarrow \mathbb{C}$.

For $f \in E$ the periodic functions

$$
W_{c}(f): E_{\tau}^{\prime} \rightarrow \mathbb{C}, \quad F \mapsto \exp \{i F(f)\}=W_{c}(f)[F]
$$

realize the commutative Weyl relations. With the commutative $*$-algebraic operations from equation (2.1) the trigonometric polynomials

$$
\Delta\left(E_{\tau}^{\prime}\right):=\operatorname{LH}\left\{W_{c}(f) \mid f \in E\right\}
$$

constitute a sub- $*$-algebra of $\left(\mathrm{C}_{b}\left(E_{\tau}^{\prime}\right),{ }_{0}\right)$.

The $\|\cdot\|_{0}$-closure of $\Delta\left(E_{\tau}^{\prime}\right)$ within the $C^{*}$-algebra $\left(\mathrm{C}_{b}\left(E_{\tau}^{\prime}\right),{ }_{0}\right)$ gives the proper sub- $C^{*}$-algebra $\operatorname{AP}\left(E_{\tau}^{\prime}\right)$ consisting of the almost periodic, $\sigma\left(E_{\tau}^{\prime}, E\right)$-continuous functions on $E_{\tau}^{\prime}$, [32, 18.2 and 33.26]. The following result from [31] ensures the independence of $\operatorname{AP}\left(E_{\tau}^{\prime}\right)$, as a $C^{*}$-algebra, from the chosen locally convex topology $\tau$.

Proposition 1. There exists a unique *-isomorphism between the commutative Weyl algebra $\mathcal{W}(E, 0)$ and $\left(\operatorname{AP}\left(E_{\tau}^{\prime}\right),{ }_{0}\right)$, which identifies the Weyl element $W^{0}(f)$ with the periodic function $W_{c}(f)$ for every $f \in E$. In this sense, $\mathcal{W}(E, 0) \cong\left(\operatorname{AP}\left(E_{\tau}^{\prime}\right),{ }_{0}\right)$, and $\Delta(E, 0) \cong \Delta\left(E_{\tau}^{\prime}\right)$.

For discussing geometric aspects we denote henceforth the phase space $E_{\tau}^{\prime}$, considered (in a loose sense, see below) as differentiable manifold with respect to the $\sigma\left(E_{\tau}^{\prime}, E\right)$-topology, by the symbol P.

One may use, as in [14], $T_{F} \mathrm{P}:=E_{\tau}^{\prime}$ as tangent space at each phase space point $F \in \mathrm{P}$. Hence, the cotangent space is given by $T_{F}^{*} \mathrm{P}:=\left(E_{\tau}^{\prime}\right)^{\prime}=E$, and its elements are identified with the test functions $f$. The total differential $d A \in T^{*} \mathrm{P}$ of $A: \mathrm{P} \rightarrow \mathbb{R}$ is defined at each $F \in \mathrm{P}$ by $d_{F} A(G):=\left.\frac{d A[c(t)]}{d t}\right|_{t=0}$ where one differentiates along all curves $t \mapsto c(t) \in \mathrm{P}$ with $c(0)=F$ and $\left.\frac{d c}{d t}\right|_{t=0}=G \in T_{F} \mathrm{P}$ (e.g. along the linear curve $t \mapsto F+t G$ for any $G \in E_{\tau}^{\prime}$ ). For a $\mathbb{C}$-valued function $A$ on $\mathrm{P}$ we put $d_{F} A:=d_{F} A_{1}+i d_{F} A_{2}$ with its real and imaginary parts, $A_{1}$ resp. $A_{2}$, which is an element of the complexified cotangent space $T_{F}^{*} \mathrm{P}+i T_{F}^{*} \mathrm{P}=:{ }^{\mathbb{C}} T_{F}^{*} \mathrm{P}$.

From now on we suppose a pre-symplectic form $\sigma$ on $E$, not necessarily $\tau$-continuous, which may be compared with a constant bivector field on the complexified cotangent bundle. With this given, a Poisson bracket $\{\cdot, \cdot\}$ may be defined

$$
\begin{aligned}
\{A, B\}[F]:= & -\sigma\left(d_{F} A_{1}, d_{F} B_{1}\right)-i \sigma\left(d_{F} A_{1}, d_{F} B_{2}\right) \\
& -i \sigma\left(d_{F} A_{2}, d_{F} B_{1}\right)+\sigma\left(d_{F} A_{2}, d_{F} B_{2}\right),
\end{aligned}
$$

a construction familiar from (finite dimensional) classical Hamiltonian mechanics, e.g. [33, 34, $35,36,37]$. The differentiability of $A$ and $B$ does not ensure the differentiability of $\mathrm{P} \ni F \mapsto$ $\{A, B\}[F] \in \mathbb{C}$. Consequently, in order to obtain a Poisson algebra we have need for a sub-*algebra $\mathcal{P}$ of $\left(\mathrm{C}_{b}(\mathrm{P}),{ }_{0}\right)$ consisting of differentiable functions, such that $\{A, B\} \in \mathcal{P}$ whenever $A, B \in \mathcal{P}$.

For the periodic function $W_{c}(f)$ from equation (2.13) we calculate

$$
d_{F} W_{c}(f)=i \exp \{i F(f)\} f=i W_{c}(f)[F] f \in \mathbb{C}_{F}^{*} \mathrm{P}, \quad \forall F \in \mathrm{P} .
$$

Insertion into the Poisson bracket (2.15) leads to

$$
\left\{W_{c}(f), W_{c}(g)\right\}=\sigma(f, g) W_{c}(f+g), \quad \forall f, g \in E .
$$


Since $\left\{W_{c}(f), W_{c}(g)\right\} \in \Delta\left(E_{\tau}^{\prime}\right)$ for every $f, g \in E$, the trigonometric polynomials $\Delta\left(E_{\tau}^{\prime}\right) \equiv \Delta(\mathrm{P})$ from equation (2.14), equipped with the Poisson bracket $\{\cdot, \cdot\}$ from equation (2.15) constitute a Poisson algebra.

By equations (2.11) and (2.17) the following consequence is immediate.

Corollary 1 (Poisson isomorphism). The *-isomorphism in Proposition 1 leads by restriction to a Poisson isomorphism from $\left(\Delta(E, 0),\{\cdot, \cdot\}_{0}\right)$ onto $\left(\Delta\left(E_{\tau}^{\prime}\right),{ }_{0},\{\cdot, \cdot\}\right)$.

The Corollary provides a belated motivation for the algebraically introduced Poisson bracket in $\left(\Delta(E, 0),\{\cdot, \cdot\}_{0}\right)$. The independence of $\tau$ is essential. Even on a flat infinite dimensional manifold there is no standard form of a differentiable structure, and various additional requirements are imposed on $\tau$ in this context. Our foregoing reasoning demonstrates that any notion of differentiability should lead to the same differentials of the classical Weyl elements and thus to the Poisson brackets (2.17).

Summary 1 (Functorial quantization for $C^{*}$-Weyl algebras). Let be given the presymplectic space $(E, \sigma)$ of any dimension with non-trivial $\sigma$, defining the Weyl relations for the abstract Weyl elements $W^{\hbar}(f), f \in E$. Associated with this input is a complex *-algebra $\Delta(E, \hbar \sigma)$, linearly spanned by the $W^{\hbar}(f)$, for any $\hbar \in \mathbb{R}$. For $\hbar=0$ (classical case) the latter admits an algebraic Poisson bracket, which conforms with that naturally induced by a differentiable structure on the $\tau$-dependent, topological dual $E_{\tau}^{\prime}$.

For $\hbar \neq 0$ (quantum regime) the non-commutative Weyl relations define scaled commutators in $\Delta(E, \hbar \sigma) . \Delta(E, \hbar \sigma)$ is easily equipped with an algebra norm, the closure with which produces the Banach-*-algebra $\overline{\Delta(E, \hbar \sigma)}^{1}$. The enveloping $C^{*}$-algebra defines the $C^{*}$-Weyl algebra $\mathcal{W}(E, \hbar \sigma)$ in this general frame.

As recapitulated in Theorem 1 the family of mappings $\left(Q_{\hbar}\right)_{\hbar \in \mathbb{R}}$, which linearly connect the classical Weyl elements with the quantized, leads to a strict deformation quantization.

Thus the whole quantization scheme depends functorially on $(E, \sigma)$.

In the Sections 3 and 6 we generalize this functorial concept of strict deformation quantization to much larger Poisson algebras. There, however, one needs beside $(E, \sigma)$, a suitable family of representations $\Pi_{\hbar}$ of $\mathcal{W}(E, \hbar \sigma)$ for $\hbar \neq 0$ and a semi-norm $\varsigma$, satisfying (3.8), as the only additional ingredients.

\section{Strict deformation quantization via Banach-*-algebras of measures}

From now on we suppose $(E, \sigma)$ to be a pre-symplectic space of infinite dimensions.

Let $I$ index the set of all finite dimensional subspaces $E_{\alpha}$ of $E$. The inductive limit topology $\tau_{\text {il }}[38,39]$ over the directed set $\left\{E_{\alpha} \mid \alpha \in I\right\}$ is the finest locally convex topology on $E$. Observe that we have chosen the finest directed set, given by all finite dimensional subspaces $E_{\alpha}$ of $E$, but for the construction of $\tau_{\text {il }}$ it would be sufficient to use any absorbing directed subset of finite dimensional subspaces.

Especially we are interested in the sub-index set $I_{\sigma}$ consisting of those indexes $\alpha \in I$ for which the restriction of the pre-symplectic form $\sigma$ is non-degenerate on $E_{\alpha}$. If $\sigma$ is non-degenerate on all of $E$, we perform the inductive limit along $I_{\sigma}$, keeping in mind $E=\bigcup_{\alpha \in I_{\sigma}} E_{\alpha},([13, \S 4])$, which leads us also to the finest locally convex topology $\tau_{\text {il }}$ on $E([39, \S$ IV.5]).

\subsection{Inductive limit of regular Borel measures}

Let us denote by $\mathrm{B}^{\tau}(E)$ the Borel subsets of $E$ with respect to the locally convex topology $\tau$ on $E$, where for $\tau=\tau_{\text {il }}$ we simply use "il" as index. By $\mathrm{B}\left(E_{\alpha}\right)$ we mean the Borel subsets of the 
finite dimensional $E_{\alpha}$ equipped with its vector space topology.

Lemma 2. If $\Lambda \in \mathrm{B}\left(E_{\alpha}\right)$, then $\Lambda \in \mathrm{B}^{\tau}(E)$. If $\Lambda \in \mathrm{B}^{\tau}(E)$, then $\Lambda \bigcap E_{\alpha} \in \mathrm{B}\left(E_{\alpha}\right)$.

The partial ordering of being "finer than" for the locally convex topologies on $E$ carries over to the partial ordering on the Borel sigma algebras given by the inclusion relation.

Proof. First note that the relative topology of $\tau$ on $E_{\alpha}$ is just its unique natural vector space topology. That is, if $\Lambda$ is an open subset of $E_{\alpha}$, then there exists a $\Lambda^{\prime} \in \tau$ with $\Lambda^{\prime} \cap E_{\alpha}=\Lambda$. $E_{\alpha}$ is a closed subset of $E$, since it contains each of its limit points, and consequently, $E_{\alpha} \in \mathrm{B}^{\tau}(E)$. The rest is immediate by the construction of the Borel sigma algebras.

Let $M^{\tau}(E)$ denote the Banach space of the finite complex Borel measures on $E$ with respect to the locally convex $\tau$ on $E$ (which are not necessarily regular as for the locally compact $E_{\alpha}$ ). The norm on $M^{\tau}(E)$ is given by the total variation $\|\mu\|_{1}:=|\mu|(E)$, where $|\mu| \in M^{\tau}(E)$ denotes the variation measure of $\mu \in M^{\tau}(E)$. (For later proofs recall that $|\mu|(\Lambda)$ is the supremum of the numbers $\sum_{k=1}^{n}\left|\mu\left(\Lambda_{k}\right)\right|$, where $\left\{\Lambda_{k} \mid k=1, \ldots, n\right\}$ ranges over all finite partitions of $\Lambda \in \mathrm{B}^{\tau}(E)$ into $\mathrm{B}^{\tau}(E)$-measurable sets [40, Section 4.1].) A Borel measure $\mu$ on $E$ is called positive - written as $\mu \geq 0-$, if $\mu(\Lambda) \geq 0$ for each $\Lambda \in \mathrm{B}^{\tau}(E)$. (Sets of positive measures will be indicated by the upper index "+".)

The finite (regular) complex Borel measures on the finite dimensional $E_{\alpha}$ are denoted by $\mathcal{M}\left(E_{\alpha}\right)$. According to Lemma 2 we may identify each $\mu \in \mathcal{M}\left(E_{\alpha}\right)$ with a measure from $M^{\tau}(E)$ concentrated on $E_{\alpha}$. The total variation norm $\|\cdot\|_{1}$ calculated on $E_{\alpha}$ coincides with that on $E$, and subsequently we understand $\mathcal{M}\left(E_{\alpha}\right)$ as a $\|\cdot\|_{1}$-closed subspace of $M^{\tau}(E)$. Since $\mathcal{M}\left(E_{\alpha}\right) \subseteq$ $\mathcal{M}\left(E_{\beta}\right)$ for $\alpha \leq \beta$, these measure spaces constitute an inductive system.

Our infinite dimensional $E$ is far from being locally compact (what each $E_{\alpha}$ is). Since each $\mu \in \mathcal{M}\left(E_{\alpha}\right)$ is a regular Borel measure on $E_{\alpha}$, we may consider the $\|\cdot\|_{1}$-closure

$$
\mathcal{M}(E):=\varlimsup_{\alpha \in I} \mathcal{M}\left(E_{\alpha}\right)\|\cdot\|_{1}
$$

as a closed subspace of $M^{\tau}(E)$. The measures in $\mathcal{M}(E)$ are regular (what we indicate by the italic $\mathcal{M}$ ) for each locally convex $\tau$, a universality property.

A further reason for considering $\mathcal{M}(E)$ instead of $M^{\tau}(E)$ lies in the applicability of Fubini's theorem in introducing associative products (see next Subsection). Note that

$$
\mathcal{M}(E)^{+}=\mathcal{M}(E) \bigcap M^{\tau}(E)^{+}=\bar{\bigcup}_{\alpha \in I} \mathcal{M}\left(E_{\alpha}\right)^{+}\left\|^{\|}\right\|_{1} .
$$

Especially it holds for $\mu \in \mathcal{M}(E)$, with Jordan decomposition $\mu=\mu_{1}-\mu_{2}+i\left(\mu_{3}-\mu_{4}\right)$, that the $\mu_{k}$ as well as $|\mu|$ are in $\mathcal{M}(E)^{+}$.

Subsequently we use the following two facts without mentioning: $\left|\int_{E} d \mu a\right| \leq \int_{E} d|\mu||a|$ for every bounded Borel measurable function $a: E \rightarrow \mathbb{C}$. If $\mu \in \mathcal{M}(E)$ and $a: E \rightarrow \mathbb{C}$ is Borel measurable with $\int_{E} d|\mu||a|<\infty$, then $\mu_{a} \in \mathcal{M}(E)$, where $d \mu_{a}(f):=a(f) d \mu(f)$.

For each $\alpha \in I$ the measure Banach space $\mathcal{M}\left(E_{\alpha}\right)$ decomposes uniquely according to

$$
\mathcal{M}\left(E_{\alpha}\right)=\mathcal{M}\left(E_{\alpha}\right)_{d} \oplus \underbrace{\mathcal{M}\left(E_{\alpha}\right)_{s} \oplus \mathcal{M}\left(E_{\alpha}\right)_{a}}_{=: \mathcal{M}\left(E_{\alpha}\right)_{c}}
$$

into three $\|\cdot\|_{1}$-closed subspaces: the discrete measures $\mathcal{M}\left(E_{\alpha}\right)_{d}$, the singularly continuous measures $\mathcal{M}\left(E_{\alpha}\right)_{s}$, and the absolutely continuous measures $\mathcal{M}\left(E_{\alpha}\right)_{a}$ with respect to the Haar measure on $E_{\alpha} \cdot \mathcal{M}\left(E_{\alpha}\right)_{c}$ are the continuous measures on $E_{\alpha}$. For the absolutely continuous measures 
one has $\mathcal{M}\left(E_{\alpha}\right)_{a} \not \subset \mathcal{M}\left(E_{\beta}\right)_{a}$ for $\alpha<\beta$, rather $\mathcal{M}\left(E_{\alpha}\right)_{a} \subseteq \mathcal{M}\left(E_{\beta}\right)_{s}$, and so they do not constitute an inductive system. Inductive systems over $I$ are obtained, however, for the discrete resp. continuous measures, since $\mathcal{M}\left(E_{\alpha}\right)_{d} \subseteq \mathcal{M}\left(E_{\beta}\right)_{d}$ resp. $\mathcal{M}\left(E_{\alpha}\right)_{c} \subseteq \mathcal{M}\left(E_{\beta}\right)_{c}$ for $\alpha \leq \beta$. So we define the $\|\cdot\|_{1}$-closed subspaces

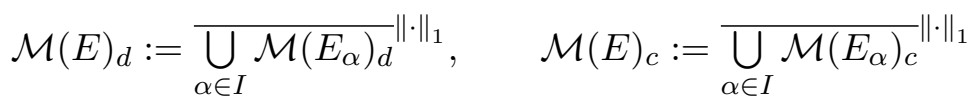

of $\mathcal{M}(E)$. We denote by $\delta(f)$ the point measure at $f \in E$, which for each $\Lambda \in \mathrm{B}^{\tau}(E)$ gives $\delta(f)(\Lambda)=1$, if $f \in \Lambda$, and $\delta(f)(\Lambda)=0$, if $f \notin \Lambda$. Each element $\mu$ of $\mathcal{M}(E)_{d}$ is of the form $\mu=$

$\sum_{k=1}^{\infty} z_{k} \delta\left(f_{k}\right)$ with $\|\mu\|_{1}=\sum_{k=1}^{\infty}\left|z_{k}\right|<\infty$ and with different $f_{k}$ 's from $E$, where $|\mu|=\sum_{k=1}^{\infty}\left|z_{k}\right| \delta\left(f_{k}\right)$ is the associated variation measure.

\subsection{Non-commutative Banach- $*$-algebras of measures}

Suppose $\hbar \in \mathbb{R}$. It is well known that for each $\alpha \in I$ the measure space $\mathcal{M}\left(E_{\alpha}\right)$ becomes a Banach-*-algebra with respect to the $*$-operation $\mu \mapsto \mu^{*}$ defined by

$$
\mu^{*}(\Lambda):=\overline{\mu(-\Lambda)}, \quad \forall \Lambda \in \mathrm{B}\left(E_{\alpha}\right)
$$

and the associative product $\star_{\hbar}$ given as the twisted resp. deformed convolution

$$
\mu \star_{\hbar} \nu(\Lambda):=\int_{E_{\alpha}} d \mu(f) \int_{E_{\alpha}} d \nu(g) \exp \left\{-\frac{i}{2} \hbar \sigma(f, g)\right\} \Lambda(f+g), \quad \forall \Lambda \in \mathrm{B}\left(E_{\alpha}\right) .
$$

$E_{\alpha} \ni h \mapsto \Lambda(h)$ denotes the characteristic function of $\Lambda$, (with $\Lambda(h)=1$ for $h \in \Lambda$, and $\Lambda(h)=0$ elsewhere). The above construction is found for the commutative case $\hbar=0$ in textbooks on measure theory and harmonic analysis, whereas for (twisted) group algebras we refer e.g. to $[41,32,42,43,44,45,46]$. The relations $\left\|\mu^{*}\right\|_{1}=\|\mu\|_{1}$ and $\left\|\mu \star_{\hbar} \nu\right\|_{1} \leq\|\mu\|_{1}\|\nu\|_{1}$ of the $*$-algebraic operations extend $\|\cdot\|_{1}$-continuously to the inductive Banach space $\mathcal{M}(E)$ from equation (3.1) making it to a Banach-*-algebra, which we denote by

$$
\left(\mathcal{M}(E), \star_{\hbar}\right)
$$

(cf. Theorem 2 below). Its identity is realized by the point measure at zero $\delta(0)$. The Banach*-algebra $\left(\mathcal{M}(E), \star_{\hbar}\right)$ is commutative, if and only if $\hbar=0$, provided that $\sigma \neq 0$.

Fubini's theorem plays the essential role in proving that the product in equation (3.4) is associative, resp. commutative for the case $\hbar=0$. Fubini's theorem, however, works well for regular Borel measures on locally compact spaces. Since our test function space $E$ has infinite dimension, $\star_{\hbar}$ may not be associative on $M^{\tau}(E)$. That is why we restrict ourselves to the regular measures $\mathcal{M}(E)$. In [13] the Banach- $*$-algebra $\left(\mathcal{M}(E), \star_{\hbar}\right)$ is performed as a purely inductive limit not taking into account that in the completion one gains further measures out of $M^{\tau}(E)$. In contradistinction to the product $\star_{\hbar}$, the $*$-involution is well defined also on $M^{\tau}(E)$ by equation (3.3).

Theorem 2. The mapping $\mathbb{R} \times \mathcal{M}(E) \times \mathcal{M}(E) \rightarrow \mathcal{M}(E),(\hbar, \mu, \nu) \mapsto \mu \star_{\hbar} \nu$ is jointly continuous (i.e., continuous with respect to the product topology on $\mathbb{R} \times \mathcal{M}(E) \times \mathcal{M}(E)$, where on $\mathcal{M}(E)$ we have the topology arising from the total variation norm $\left.\|\cdot\|_{1}\right)$.

Proof. For each $\mu, \nu \in \mathcal{M}(E)$ and all $\hbar, \lambda \in \mathbb{R}$ we obtain

$$
\left|\left(\mu \star_{\hbar} \nu-\mu \star_{\lambda} \nu\right)(\Lambda)\right|
$$




$$
\begin{aligned}
& =\left|\int_{E} d \mu(f) \int_{E} d \nu(g)\left(\exp \left\{-\frac{i}{2} \hbar \sigma(f, g)\right\}-\exp \left\{-\frac{i}{2} \lambda \sigma(f, g)\right\}\right) \Lambda(f+g)\right| \\
& \leq \int_{E} d|\mu|(f) \int_{E} d|\nu|(g)\left|\exp \left\{-\frac{i}{2} \hbar \sigma(f, g)\right\}-\exp \left\{-\frac{i}{2} \lambda \sigma(f, g)\right\}\right| \Lambda(f+g),
\end{aligned}
$$

from which we conclude

$$
\left|\mu \star_{\hbar} \nu-\mu \star_{\lambda} \nu\right|(\Lambda) \leq \int_{E} d|\mu|(f) \int_{E} d|\nu|(g)\left|\exp \left\{-\frac{i}{2} \hbar \sigma(f, g)\right\}-\exp \left\{-\frac{i}{2} \lambda \sigma(f, g)\right\}\right| \Lambda(f+g)
$$

for all $\Lambda \in \mathrm{B}^{\tau}(E)$. Taking into account $\|\mu\|_{1}=|\mu|(E)$ for $\mu \in \mathcal{M}(E)$ yields that

$$
\left\|\mu \star_{\hbar} \nu-\mu \star_{\lambda} \nu\right\|_{1} \leq \int_{E} d|\mu|(f) \int_{E} d|\nu|(g)\left|\exp \left\{-\frac{i}{2} \hbar \sigma(f, g)\right\}-\exp \left\{-\frac{i}{2} \lambda \sigma(f, g)\right\}\right| \stackrel{\hbar \rightarrow \lambda}{\longrightarrow} 0 .
$$

Now the result follows from standard $\|\cdot\|_{1}$-estimations.

The $*$-algebraic structure (consisting of the $*$-operation and the product $\star_{\hbar}$ ) allows further concept of positivity. For a measure $\mu \in \mathcal{M}(E)^{+}$its positivity is for no $\hbar \in \mathbb{R}$ connected with the $*$-algebraic positivity. Recall that the latter means $\mu=\nu^{*} \star \hbar \nu$ for some $\nu \in \mathcal{M}(E)$. The positive measures $\mathcal{M}(E)^{+}$, however, constitute a closed convex cone in $\mathcal{M}(E)$ satisfying $\mathcal{M}(E)^{+} \bigcap\left(-\mathcal{M}(E)^{+}\right)=\{0\}$ (pointedness) and $\mathcal{M}(E)^{+} \star_{0} \mathcal{M}(E)^{+} \subseteq \mathcal{M}(E)^{+}$.

For each $\alpha \in I$ we have that $\mathcal{M}\left(E_{\alpha}\right)_{d}$ is a sub-Banach-*-algebra, whereas $\mathcal{M}\left(E_{\alpha}\right)_{a}$ and $\mathcal{M}\left(E_{\alpha}\right)_{c}$ constitute closed (two-sided) $*$-ideals of the Banach- $*$-algebra $\left(\mathcal{M}\left(E_{\alpha}\right), \star_{\hbar}\right)$, (e.g. [32]). Thus also in the inductive limit the discrete measures $\mathcal{M}(E)_{d}$ constitute a sub-Banach-*algebra and the continuous measures $\mathcal{M}(E)_{c}$ a closed (two-sided) *-ideal of the Banach-*algebra $\left(\mathcal{M}(E), \star_{\hbar}\right)$. Especially $\mathcal{M}(E)_{d f}$, the subspace of measures in $\mathcal{M}(E)_{d}$ with finite support (for which $\sum_{k} z_{k} \delta\left(f_{k}\right)$ ranges over a finite number of terms), is a $\|\cdot\|_{1}$-dense sub-*-algebra of $\left(\mathcal{M}(E)_{d}, \star_{\hbar}\right)$.

The following result is immediate with the construction in Subsection 2.2.

Proposition 2 (Measure realization of the Weyl algebra). Let $\hbar \in \mathbb{R}$, and let $(E, \sigma)$ be an arbitrary pre-symplectic space. If we associate for each $f \in E$ the abstract Weyl element $W^{\hbar}(f)$ with the point measure $\delta(f)$, the Weyl relations

$$
W^{\hbar}(f) W^{\hbar}(g)=\exp \left\{-\frac{i}{2} \hbar \sigma(f, g)\right\} W^{\hbar}(f+g), \quad W^{\hbar}(f)^{*}=W^{\hbar}(-f), \quad \forall f, g \in E,
$$

from equation (2.7) are transformed into

$$
\delta(f) \star_{\hbar} \delta(g)=\exp \left\{-\frac{i}{2} \hbar \sigma(f, g)\right\} \delta(f+g), \quad \delta(f)^{*}=\delta(-f), \quad \forall f, g \in E .
$$

Moreover, the *-algebra $\Delta(E, \hbar \sigma)$ is bijectively transformed onto the $*$-algebra $\left(\mathcal{M}(E)_{d f}, \star_{\hbar}\right)$. Closure in the $\|\cdot\|_{1}$-norm leads to the Banach-*-algebra $\overline{\Delta(E, \hbar \sigma)}^{1}=\left(\mathcal{M}(E)_{d}, \star_{\hbar}\right)$, for which the enveloping $C^{*}$-algebra is $*$-isomorphic to $\overline{\Delta(E, \hbar \sigma)}\|\cdot\|=\mathcal{W}(E, \hbar \sigma)$, the closure in the $C^{*}$-norm.

Thus we may identify the boson field algebra $\mathcal{W}(E, \hbar \sigma)$ with the enveloping $C^{*}$-algebra of the discrete measure algebra $\left(\mathcal{M}(E)_{d}, \star_{\hbar}\right)$.

\subsection{Poisson algebras of measures}

Since $\tau_{\text {il }}$ is the finest locally convex topology on $E$, every semi-norm $\kappa$ on $E$ is $\tau_{\text {il }}$-continuous. Thus from $\mathcal{M}(E) \subseteq M^{\mathrm{il}}(E)$ it follows that the integral $\int_{E} \kappa(f)^{m} d|\mu|(f)$ is well defined for all $\mu \in \mathcal{M}(E)$ with respect to the Borel sigma algebra $\mathrm{B}^{\mathrm{il}}(E)$, but possibly leads to the value $\infty$. If $\kappa$ is a $\tau$-continuous semi-norm with respect to any other locally convex topology $\tau$ on $E$, then the integral $\int_{E} \kappa(f)^{m} d|\mu|(f)$ may be understood also in terms of the Borel sigma algebra $\mathrm{B}^{\tau}(E)$. 
Let $n \in \mathbb{N} \bigcup\{0, \infty\}$. We define for each semi-norm $\kappa$ on $E$ the space

$$
\mathcal{M}_{\kappa}^{n}(E):=\left\{\mu \in \mathcal{M}(E)\left|\int_{E} \kappa(f)^{m} d\right| \mu \mid(f)<\infty \text { for all } 0 \leq m \leq n\right\} .
$$

We call $\mu_{\kappa}^{m} \in \mathcal{M}(E)$ with $d \mu_{\kappa}^{m}(f):=\kappa(f)^{m} d \mu(f)$ the $m$-th moment measure for $\mu \in \mathcal{M}_{\kappa}^{n}(E)$ with respect to the semi-norm $\kappa$. Note that $\mu \in \mathcal{M}_{\kappa}^{n}(E)$ implies $|\mu| \in \mathcal{M}_{\kappa}^{n}(E)$, and $\left|\mu_{\kappa}^{m}\right|=|\mu|_{\kappa}^{m}$ by definition. For finite $n \in \mathbb{N}$ it is easily shown that $\mathcal{M}_{\kappa}^{n}(E)$ is a Banach space with respect to the norm

$$
\|\mu\|_{\kappa}^{n}:=\|\mu\|_{1}+\sum_{m=1}^{n}\left\|\mu_{\kappa}^{m}\right\|_{1} .
$$

$\mathcal{M}_{\kappa}^{\infty}(E)=\bigcap_{n} \mathcal{M}_{\kappa}^{n}(E)$ turns out to be a Fréchet space with respect to the metrizable locally convex topology $v_{\kappa}$ arising from the increasing system of norms $\|\cdot\|_{\kappa}^{n}, n \in \mathbb{N}$. For $n=0$ we re-obtain $\mathcal{M}_{\kappa}^{0}(E)=\mathcal{M}(E)$ and $\|\cdot\|_{\kappa}^{0}=\|\cdot\|_{1}$. Obviously, $\bigcup_{\alpha \in I} \mathcal{M}_{\kappa}^{n}\left(E_{\alpha}\right)$ is dense in $\mathcal{M}_{\kappa}^{n}(E)$ with respect to $\|\cdot\|_{\kappa}^{n}$, for $n<\infty$, resp. to $v_{\kappa}$, for $n=\infty$.

Lemma 3. For each $n \in \mathbb{N} \bigcup\{\infty\}$, each $\hbar \in \mathbb{R}$, and each semi-norm $\kappa$ on the pre-symplectic space $E$ the previously introduced linear space of complex measures $\mathcal{M}_{\kappa}^{n}(E)$ is a sub-*-algebra of the Banach-*-algebra $\left(\mathcal{M}(E), \star_{\hbar}\right)$. The following assertions are valid:

(a) For each $n \in \mathbb{N}\|\mu\|_{\kappa}^{n}=\left\|\mu^{*}\right\|_{\kappa}^{n}$ and $\left\|\mu \star_{\hbar} \nu\right\|_{\kappa}^{n} \leq c_{n}\|\mu\|_{\kappa}^{n}\|\nu\|_{\kappa}^{n}$ for all $\mu, \nu \in \mathcal{M}_{\kappa}^{n}(E)$ with some constant $c_{n} \geq 1$ defined in equation (3.6) below. (One has $c_{1}=1$ for $n=1$ but necessarily $c_{n}>1$ for $n \geq 2$, and so $\mathcal{M}_{\kappa}^{n}(E)$ is not a Banach*-algebra with respect to $\|\cdot\|_{\kappa}^{n}$ but with respect to an equivalent norm.)

(b) $\mathcal{M}_{\kappa}^{\infty}(E)$ is a Fréchet-*-algebra with respect to its Fréchet topology $v_{\kappa}$ (i.e., the product is jointly $v_{\kappa}$-continuous, and the $*$-operation is $v_{\kappa}$-continuous).

Proof. $|\mu|^{*}(\Lambda)=\left|\mu^{*}\right|(\Lambda)=|\mu|(-\Lambda)$ for all $\Lambda \in \mathrm{B}^{\mathrm{il}}(E)$ together with the semi-norm property $\kappa(f)=\kappa(-f)$ imply $\mathcal{M}_{\kappa}^{n}(E)$ to be invariant under the $*$-operation $\mu \mapsto \mu^{*}$ and $\|\mu\|_{\kappa}^{n}=\left\|\mu^{*}\right\|_{\kappa}^{n}$.

Let $\mu, \nu \in \mathcal{M}_{\kappa}^{n}(E)$. Then $\mu \star_{\hbar} \nu \in \mathcal{M}(E)$. We show $\mu \star_{\hbar} \nu \in \mathcal{M}_{\kappa}^{n}(E)$. From the semi-norm property $\kappa(f+g) \leq \kappa(f)+\kappa(g)$ we obtain for all $m \in \mathbb{N}$ that

$$
\begin{aligned}
\left|\left(\mu \star_{\hbar} \nu\right)_{\kappa}^{m}(\Lambda)\right| & =\left|\int_{E} d \mu(f) \int_{E} d \nu(g) \exp \left\{-\frac{i}{2} \hbar \sigma(f, g)\right\} \kappa(f+g)^{m} \Lambda(f+g)\right| \\
& \leq \int_{E} d|\mu|(f) \int_{E} d|\nu|(g)(\kappa(f)+\kappa(g))^{m} \Lambda(f+g)=\sum_{k=0}^{m}\left(\begin{array}{c}
m \\
k
\end{array}\right)|\mu|_{\kappa}^{m-k} \star_{0}|\nu|_{\kappa}^{k}(\Lambda) .
\end{aligned}
$$

Thus, $\left|\mu \star_{\hbar} \nu\right|_{\kappa}^{m} \leq \sum_{k=0}^{m}\left(\begin{array}{c}m \\ k\end{array}\right)|\mu|_{\kappa}^{m-k} \star_{0}|\nu|_{\kappa}^{k}$, resp. $\left\|\left(\mu \star_{\hbar} \nu\right)_{\kappa}^{m}\right\|_{1} \leq \sum_{k=0}^{m}\left(\begin{array}{c}m \\ k\end{array}\right)\left\|\mu_{\kappa}^{m-k}\right\|_{1}\left\|\nu_{\kappa}^{k}\right\|_{1}<\infty$ for all $m \leq n$. Consequently, $\mu \star_{\hbar} \nu \in \mathcal{M}_{\kappa}^{n}(E)$. Moreover,

$$
\begin{aligned}
\left\|\mu \star_{\hbar} \nu\right\|_{\kappa}^{n} & =\sum_{m=0}^{n}\left\|\left(\mu \star_{\hbar} \nu\right)_{\kappa}^{m}\right\|_{1} \leq \sum_{m=0}^{n} \sum_{k=0}^{m}\left(\begin{array}{c}
m \\
k
\end{array}\right)\left\|\mu_{\kappa}^{m-k}\right\|_{1}\left\|\nu_{\kappa}^{k}\right\|_{1} \\
& \leq \underbrace{\sup \left\{\left(\begin{array}{c}
n \\
k
\end{array}\right) \mid k=0,1, \ldots, n\right\}}_{=: c_{n}}\|\mu\|_{\kappa}^{n}\|\nu\|_{\kappa}^{n} .
\end{aligned}
$$

Hence part (a) is proved, which also leads to proving of the part (b).

For each fixed $n \in \mathbb{N} \bigcup\{\infty\}$ the moment measure spaces $\mathcal{M}_{\kappa}^{n}(E)$ are in inverse-orderpreserving correspondence with the semi-norms $\kappa$ on $E$ : If the semi-norm $\eta$ is stronger than $\kappa-$ i.e., $0 \leq \kappa \leq c \eta$ for some $c \geq 0$-, then $\mathcal{M}(E) \supseteq \mathcal{M}_{\kappa}^{n}(E) \supseteq \mathcal{M}_{\eta}^{n}(E)$, where $\mathcal{M}(E)$ corresponds 
to the trivial semi-norm. If two semi-norms $\kappa$ and $\eta$ are equivalent $-c_{1} \eta \leq \kappa \leq c_{2} \eta$ with some constant $0<c_{1} \leq c_{2}-$, then $\mathcal{M}_{\kappa}^{n}(E)=\mathcal{M}_{\eta}^{n}(E)$. For a collection of semi-norms $\kappa_{\beta}, \beta \in B$, the intersection $\bigcap_{\beta \in B} \mathcal{M}_{\kappa_{\beta}}^{n}(E)$ gives a further sub-*-algebra of $\left(\mathcal{M}(E), \star_{\hbar}\right)$.

Analogously to equation (2.2) we introduce for each $\hbar \neq 0$ the scaled commutator

$$
\{\mu, \nu\}_{\hbar}:=\frac{i}{\hbar}\left(\mu \star_{\hbar} \nu-\nu \star_{\hbar} \mu\right), \quad \forall \mu, \nu \in \mathcal{M}(E), \quad \hbar \neq 0 .
$$

For $\hbar=0$, instead of a commutator, we construct a Poisson bracket $\{\cdot, \cdot\}_{0}$. The starting point for this construction is a semi-norm $\varsigma$ on $E$ satisfying

$$
|\sigma(f, g)| \leq c \varsigma(f) \varsigma(g), \quad \forall f, g \in E,
$$

for some constant $c>0$. Whenever we write $\varsigma$ below, we assume this inequality. Since each semi-norm is continuous with respect to the finest locally convex topology $\tau_{\mathrm{il}}$, we conclude that a semi-norm $\varsigma$ satisfying equation (3.8) exists, if and only if the pre-symplectic form $\sigma$ is jointly continuous with respect to some locally convex topology $\tau$ on $E$. Clearly, $\varsigma$ has to be a norm on $E$, if $\sigma$ is non-degenerate on $E$.

Theorem 3. For every $\mu, \nu \in \mathcal{M}_{\varsigma}^{1}(E)$ the expression

$$
\{\mu, \nu\}_{0}(\Lambda):=\int_{E} d \mu(f) \int_{E} d \nu(g) \sigma(f, g) \Lambda(f+g), \quad \forall \Lambda \in \mathrm{B}^{\mathrm{il}}(E),
$$

gives a well-defined measure $\{\mu, \nu\}_{0} \in \mathcal{M}(E)$. Furthermore, if $\mu, \nu \in \mathcal{M}_{\varsigma}^{n}(E)$ for some finite $n \in \mathbb{N}$, then $\{\mu, \nu\}_{0} \in \mathcal{M}_{\varsigma}^{n-1}(E)$, and

$$
\left\|\{\mu, \nu\}_{0}\right\|_{\varsigma}^{n-1} \leq c c_{n-1}\|\mu\|_{\varsigma}^{n}\|\nu\|_{\varsigma}^{n}, \quad \forall \mu, \nu \in \mathcal{M}_{\varsigma}^{n}(E) .
$$

With the scaled commutators $\{\cdot, \cdot\}_{\hbar}$ from equation $(3.7)$ for $\hbar \neq 0$, and the bracket $\{\cdot, \cdot\}_{0}$ for $\hbar=0$, the mapping

$$
\mathbb{R} \times \mathcal{M}_{\varsigma}^{n}(E) \times \mathcal{M}_{\varsigma}^{n}(E) \rightarrow \mathcal{M}_{\varsigma}^{n-1}(E), \quad(\hbar, \mu, \nu) \mapsto\{\mu, \nu\}_{\hbar}
$$

is jointly continuous for every $n \in \mathbb{N} \bigcup\{\infty\}$, that means, continuous with respect to the product topology on $\mathbb{R} \times \mathcal{M}_{\varsigma}^{n}(E) \times \mathcal{M}_{\varsigma}^{n}(E)$ arising from the norm $\|\cdot\|_{\varsigma}^{n}$ and the norm $\|\cdot\|_{\varsigma}^{n-1}$ on $\mathcal{M}_{\varsigma}^{n-1}(E)$ for $n<\infty$, resp. the Fréchet topology $v_{\kappa}$ for $n=\infty$.

Furthermore, the mapping

$$
\{\cdot, \cdot\}_{0}: \mathcal{M}_{\varsigma}^{\infty}(E) \times \mathcal{M}_{\varsigma}^{\infty}(E) \rightarrow \mathcal{M}_{\varsigma}^{\infty}(E), \quad(\mu, \nu) \mapsto\{\mu, \nu\}_{0}
$$

defines a jointly continuous Poisson bracket $\{\cdot, \cdot\}_{0}$ with respect to the Fréchet topology $v_{\varsigma}$, which makes $\left(\mathcal{M}_{\varsigma}^{\infty}(E), \star_{0},\{\cdot, \cdot\}_{0}\right)$ to a Poisson algebra. Suppose $\mathcal{P}$ to be one of the following cases:

(I) $\mathcal{P}=\mathcal{M}_{\kappa}^{\infty}(E)$ with respect to some semi-norm $\kappa$ on $E$ stronger than, or equivalent to $\varsigma$.

(II) $\mathcal{P}=\bigcap_{\beta \in B} \mathcal{M}_{\kappa_{\beta}}^{\infty}(E)$ for a collection of semi-norms $\kappa_{\beta}, \beta \in B$, satisfying $\varsigma \leq \sum_{\beta \in \Gamma} c_{\beta} \kappa_{\beta}$ for some finite subindex set $\Gamma \subseteq B$ and some constants $c_{\beta}>0$.

(III) $\mathcal{P}$ is the intersection of $\mathcal{M}(E)_{d}=\overline{\Delta(E, 0)}^{1}$ or of $\mathcal{M}(E)_{c}$ with one of the above cases. One may also take intersections with the absolutely continuous complex measures $\mathcal{M}\left(E_{\alpha}\right)_{a}$ on a finite dimensional subspace $E_{\alpha}$ of $E$.

$(I V) \mathcal{P}=\mathcal{M}(E)_{d f}=\Delta(E, 0)$.

Then $\mathcal{P} \subseteq \mathcal{M}_{\varsigma}^{\infty}(E)$, and $\left(\mathcal{P}, \star_{0},\{\cdot, \cdot\}_{0}\right)$ constitutes a Poisson algebra, which in addition is invariant under the product $\star_{\hbar}$ for each $\hbar \in \mathbb{R}$. 
Proof. Estimation (3.8) ensures that for all $\Lambda \in \mathrm{B}^{\mathrm{il}}(E)$ in equation (3.9) we have that

$$
\begin{aligned}
\left|\left(\{\mu, \nu\}_{0}\right)_{\varsigma}^{m}(\Lambda)\right| & \leq \int_{E} d|\mu|(f) \int_{E} d|\nu|(g) \varsigma(f+g)^{m}|\sigma(f, g)| \Lambda(f+g) \\
& \leq c \int_{E} d|\mu|(f) \int_{E} d|\nu|(g)(\varsigma(f)+\varsigma(g))^{m} \varsigma(f) \varsigma(g) \Lambda(f+g) \\
& \leq c \sum_{k=0}^{m}\left(\begin{array}{c}
m \\
k
\end{array}\right) \int_{E} d|\mu|_{\varsigma}^{m-k+1}(f) \int_{E} d|\nu|_{\varsigma}^{k+1}(g) \Lambda(f+g),
\end{aligned}
$$

implying $\left\|\left(\{\mu, \nu\}_{0}\right)_{\varsigma}^{m}\right\|_{1} \leq c \sum_{k=0}^{m}\left(\begin{array}{c}m \\ k\end{array}\right)\left\|\mu_{\varsigma}^{m-k+1}\right\|_{1}\left\|\nu_{\varsigma}^{k+1}\right\|_{1}$. Thus

$$
\begin{aligned}
\left\|\{\mu, \nu\}_{0}\right\|_{\varsigma}^{n-1} & =\sum_{m=0}^{n-1}\left\|\left(\{\mu, \nu\}_{0}\right)_{\varsigma}^{m}\right\|_{1} \leq c \sum_{m=0}^{n-1} \sum_{k=0}^{m}\left(\begin{array}{c}
m \\
k
\end{array}\right)\left\|\mu_{\varsigma}^{m-k+1}\right\|_{1}\left\|\nu_{\varsigma}^{k+1}\right\|_{1} \\
\leq c & \underbrace{\sup \left\{\left(\begin{array}{c}
n-1 \\
k
\end{array}\right) \mid k=0,1, \ldots, n-1\right\}}_{\left.=c_{n-1} \quad \text { (defined in equation }(3.6)\right)}\|\mu\|_{\varsigma}^{n}\|\nu\|_{\varsigma}^{n} .
\end{aligned}
$$

Thus $\{\mu, \nu\}_{0} \in \mathcal{M}_{\varsigma}^{n-1}(E)$, whenever $\mu, \nu \in \mathcal{M}_{\varsigma}^{n}(E)$.

We now turn to the joint continuity in (3.10). We demonstrate here only the case $n=1$, for $n \geq 2$ one may proceed similarly as in the previous argumentations. Let $\mu, \nu \in \mathcal{M}_{\varsigma}^{1}(E)$. Then

$$
\begin{aligned}
& \left|\left(\{\mu, \nu\}_{\hbar}-\{\mu, \nu\}_{0}\right)(\Lambda)\right| \leq\left|\{\mu, \nu\}_{\hbar}-\{\mu, \nu\}_{0}\right|(\Lambda) \\
& \quad \leq \int_{E} d|\mu|(f) \int_{E} d|\nu|(g)\left|\frac{i}{\hbar}\left(\exp \left\{-\frac{i}{2} \hbar \sigma(f, g)\right\}-\exp \left\{\frac{i}{2} \hbar \sigma(f, g)\right\}\right)-\sigma(f, g)\right| \Lambda(f+g) .
\end{aligned}
$$

Inserting $\Lambda=E$ finally gives $\lim _{\hbar \rightarrow 0}\left\|\{\mu, \nu\}_{\hbar}-\{\mu, \nu\}_{0}\right\|_{1}=0$ by use of the differential quotient limit $\lim _{\hbar \rightarrow 0} \frac{i}{\hbar}\left(\exp \left\{-\frac{i}{2} \hbar \sigma(f, g)\right\}-\exp \left\{\frac{i}{2} \hbar \sigma(f, g)\right\}\right)=\sigma(f, g)$. Note that by the mean value theorem of differential calculus with one real variable we obtain a bound by

$$
\left|\frac{\exp \left\{ \pm \frac{i}{2} \hbar \sigma(f, g)\right\}-1}{\hbar}\right| \leq \frac{1}{2}|\sigma(f, g)| \leq \frac{c}{2} \varsigma(f) \varsigma(g), \quad \forall 0 \neq \hbar \in \mathbb{R}, \quad \forall f, g \in E,
$$

which allows application of Lebesgue's dominated convergence theorem. Consequently,

$$
\begin{aligned}
\left\|\{\mu, \nu\}_{\hbar}-\left\{\mu^{\prime}, \nu^{\prime}\right\}_{0}\right\|_{1} & \leq\left\|\{\mu, \nu\}_{\hbar}-\{\mu, \nu\}_{0}\right\|_{1}+\left\|\left\{\mu-\mu^{\prime}, \nu\right\}_{0}\right\|_{1}+\left\|\left\{\mu^{\prime}, \nu-\nu^{\prime}\right\}_{0}\right\|_{1} \\
& \leq\left\|\{\mu, \nu\}_{\hbar}-\{\mu, \nu\}_{0}\right\|_{1}+\left\|\left(\mu-\mu^{\prime}\right)_{\varsigma}^{1}\right\|_{1}\left\|\nu_{\varsigma}^{1}\right\|_{1}+\left\|\mu_{\varsigma}^{\prime 1}\right\|_{1}\left\|\left(\nu-\nu^{\prime}\right)_{\varsigma}^{1}\right\|_{1},
\end{aligned}
$$

implying the joint continuity at $\hbar=0$. Now the rest is easily shown.

Of course, one obtains a larger Poisson algebra than $\mathcal{M}_{\varsigma}^{\infty}(E)$, if one finds a semi-norm $\varsigma^{\prime}$, which is smaller than $\varsigma$ but also satisfies the estimate (3.8).

\subsection{Strict deformation quantization of Banach-*-type}

With our previous results we arrive at a version of strict deformation quantization, which is formulated in terms of our measure Banach-*-algebras $\left(\mathcal{M}(E), \star_{\hbar}\right)$, instead of $C^{*}$-algebras as in Definition 1. In some sense, the basic idea of deformation quantization is realized in this version more exactly than in the $C^{*}$-algebraic manner: One has the same mathematical quantities for observables in the classical and quantum regime. One has even the same topology in both cases, given by the $\|\cdot\|_{1}$-norm, what makes the classical limit easier. The classical product is now the usual convolution and the quantum product is the twisted convolution. 


\section{Theorem 4 (Functorial Banach-*-strict deformation quantization).}

Theorem 2 implies von Neumann's condition

$$
\lim _{\hbar \rightarrow 0}\left\|\mu \star_{\hbar} \nu-\mu \star_{0} \nu\right\|_{1}=0, \quad \forall \mu, \nu \in \mathcal{M}(E) .
$$

From Theorem 3 follows Dirac's condition

$$
\lim _{\hbar \rightarrow 0}\left\|\{\mu, \nu\}_{\hbar}-\{\mu, \nu\}_{0}\right\|_{1}=0, \quad \forall \mu, \nu \in \mathcal{M}_{\varsigma}^{1}(E) .
$$

Rieffel's condition is trivially fulfilled, since on all the Banach-*-algebras $\left(\mathcal{M}(E), \star_{\hbar}\right), \hbar \in \mathbb{R}$, we have the same norm, namely the total variation norm $\|\cdot\|_{1}$.

Now select an arbitrary Poisson algebra $\left(\mathcal{P}, \star_{0},\{\cdot, \cdot\}_{0}\right)$ from Theorem 3 and define for each $\hbar \in \mathbb{R}$ the identical (thus injective) embedding

$$
Q_{\hbar}^{B}: \mathcal{P} \longrightarrow(\mathcal{M}(E), \star \hbar), \quad \mu \longmapsto \mu
$$

as quantization map. Then the image $Q_{\hbar}^{B}(\mathcal{P})$ is a sub-*-algebra of $\left(\mathcal{M}(E), \star_{\hbar}\right)$ for every $\hbar \in \mathbb{R}$.

Summarizing it follows that $\left(Q_{\hbar}^{B}\right)_{\hbar \in \mathbb{R}}$ constitutes a Banach-*-algebra version of strict deformation quantization, which depends functorially on the pre-symplectic space $(E, \sigma)$, provided the semi-norms are chosen, which characterize the enlarged measure Poisson algebras.

\section{Poisson algebras of phase space functions}

By means of Fourier transformation we are going to realize the measure Poisson algebras $\left(\mathcal{P}, \star_{0},\{\cdot, \cdot\}_{0}\right)$ from Subsection 3.3 (see especially Theorem 3) in terms of functions on $E_{\tau}^{\prime}$, the $\tau$-dual of $E$. For $\tau=\tau_{\text {il }}$ it coincides with the space $E^{\prime}$ of all $\mathbb{R}$-linear functionals on $E$.

\subsection{Fourier transformation of the measure algebras}

Lemma 4. The partial ordering of being "finer than" of the locally convex topologies on E carries over to the partial ordering of the dual spaces given by inclusion. Furthermore, if $\rho \leq \tau$, then $E_{\rho}^{\prime}$ is dense in $E_{\tau}^{\prime}$ with respect to the $\sigma\left(E_{\tau}^{\prime}, E\right)$-topology.

Proof. The $\sigma\left(E_{\tau}^{\prime}, E\right)$-dual of $E_{\tau}^{\prime}$ is $E$ itself, and thus consists of the functionals $F \mapsto F(f)$, $f \in E$. Since $\rho$ is Hausdorff, it holds that $F(g)=0$ for all $F \in E_{\rho}^{\prime}$ implies $g=0$. So the "annihilator" of $E_{\rho}^{\prime}$ vanishes, and thus $E_{\rho}^{\prime}$ is dense in $E_{\tau}^{\prime}$ according to the Hahn-Banach theorem [39, Corollary IV.3.14].

For every locally convex $\tau$ the Fourier transformation $\mathbb{F}$, acting on all finite Borel measures $M^{\tau}(E)$, is defined

$$
\mathbb{F} \mu[F] \equiv \widehat{\mu}[F]:=\int_{E} d \mu(f) \exp \{i F(f)\}, \quad F \in E_{\tau}^{\prime}, \quad \mu \in M^{\tau}(E) .
$$

In terms of the Weyl functions $W_{c}(f)[F]=\exp \{i F(f)\}, f \in E$, the Fourier transformation writes

$$
\widehat{\mu}=\int_{E} d \mu(f) W_{c}(f), \quad \mu \in M^{\tau}(E)
$$

and may now be read as an integral over phase space functions.

For $\alpha \in I$ a measure $\mu \in \mathcal{M}\left(E_{\alpha}\right)$ is concentrated on $E_{\alpha}$, and hence its Fourier transform satisfies $\widehat{\mu}[F]=\widehat{\mu}[G]$ for all $F, G \in E_{\tau}^{\prime}$ with $F(f)=G(f) \forall f \in E_{\alpha}$. This is just the identification 
of the dual $E_{\alpha}^{\prime}$ of $E_{\alpha}$ with classes in $E_{\tau}^{\prime}$. In this sense we identify subsequently $E_{\alpha}^{\prime}$ with a part of $E_{\tau}^{\prime}$.

We denote the Fourier image of a set of measures by a hat, indicating by $\left(E_{\tau}^{\prime}\right)$ the independent variables. Thus we write e.g. $\widehat{M}\left(E_{\tau}^{\prime}\right):=\mathbb{F} M^{\tau}(E)$. We know $\widehat{M}\left(E_{\tau}^{\prime}\right) \subseteq \mathrm{C}_{b}\left(E_{\tau}^{\prime}\right)$. For the point measures one has $\widehat{\delta(f)}=W_{c}(f)$. Thus we obtain the following subspace structure for some Fourier transformed measure spaces

$$
\widehat{\mathcal{M}}\left(E_{\tau}^{\prime}\right)_{d f} \subset \widehat{\mathcal{M}}\left(E_{\tau}^{\prime}\right)_{d} \subset \operatorname{AP}\left(E_{\tau}^{\prime}\right) \subset \mathrm{C}_{b}\left(E_{\tau}^{\prime}\right),
$$

where the *-algebra $\widehat{\mathcal{M}}\left(E_{\tau}^{\prime}\right)_{d f}=\Delta\left(E_{\tau}^{\prime}\right)$ of trigonometric functions and the $C^{*}$-algebra of all almost periodic functions $\mathrm{AP}\left(E_{\tau}^{\prime}\right)$ are introduced in Subsection 2.5. They are independent of $\tau$ as algebras. In the set of special almost periodic functions $\widehat{\mathcal{M}}\left(E_{\tau}^{\prime}\right)_{d}$, one can consider the $\|\cdot\|_{1}$-norm, making it $*$-isomorphic to the $\tau$-independent Banach-*-algebra $\overline{\Delta(E, \hbar \sigma)}^{1}$ of Subsection 2.2.

Since the Fourier transforms are $\sigma\left(E_{\tau}^{\prime}, E\right)$-continuous functions, we conclude from the above Lemma that the sup-norm may be evaluated on smaller dual spaces,

$$
\|\widehat{\mu}\|_{0}=\sup \left\{|\widehat{\mu}[F]| \mid F \in E_{\tau}^{\prime}\right\}=\sup \left\{|\widehat{\mu}[F]| \mid F \in E_{\rho}^{\prime}\right\}, \quad \forall \mu \in M^{\tau}(E),
$$

where $\rho$ is any locally convex topology on $E$ with $\rho \leq \tau$.

Because of the mentioned universality of $\mathcal{M}(E)$ we have

$$
\widehat{\mathcal{M}}\left(E_{\tau}^{\prime}\right)_{d} \subset \mathbb{F} \mathcal{M}(E)=\widehat{\mathcal{M}}\left(E_{\tau}^{\prime}\right) \subset \mathrm{C}_{b}\left(E_{\tau}^{\prime}\right),
$$

for every locally convex topology $\tau$ on $E$. This means that the extension of the considered phase space functions from $\widehat{\mathcal{M}}\left(E_{\tau}^{\prime}\right)_{d}$ to $\widehat{\mathcal{M}}\left(E_{\tau}^{\prime}\right)$ depends functorially on $E$. A concrete topology $\tau$ comes into play only if the measures $\mu \in M^{\tau}(E)$ are considered which are not in $\mathcal{M}(E)$, respectively, which possess Fourier transforms $\widehat{\mu} \in \widehat{M}\left(E_{\tau}^{\prime}\right)$ not contained in $\widehat{\mathcal{M}}\left(E_{\tau}^{\prime}\right)$.

If we use in $\widehat{M}\left(E_{\tau}^{\prime}\right)$ the *-operation of pointwise complex conjugation inherited from $\mathrm{C}_{b}\left(E_{\tau}^{\prime}\right)$ (see equation $(2.1)$ ), then the Fourier transformation $\mathbb{F}$ is $*$-preserving, that is, $\widehat{\mu^{*}}=\widehat{\mu}^{*}$ for all $\mu \in M^{\tau}(E)$. The Fourier transformation of the deformed resp. twisted convolution $\star_{\hbar}$ from equation (3.4) leads to the deformed product ${ }^{\hbar}$ on $\widehat{\mathcal{M}}\left(E_{\tau}^{\prime}\right)$,

$$
\widehat{\mu} \cdot \hbar \widehat{\nu}:=\widehat{\mu \star_{\hbar} \nu}, \quad \forall \mu, \nu \in \mathcal{M}(E), \quad \hbar \in \mathbb{R} .
$$

This agrees with the deformed product from equation (2.3) arising from a strict deformation quantization (cf. also Subsection 1.1). By construction it follows for each $\hbar \in \mathbb{R}$ that $\mathbb{F}$ is a *-isomorphism from the Banach-*-algebra $\left(\mathcal{M}(E), \star_{\hbar}\right)$ onto the $*$-algebra $\left(\widehat{\mathcal{M}}\left(E_{\tau}^{\prime}\right),{ }^{\circ}\right)$ consisting of functions.

The Weyl relations (3.5) for the point measures $\delta(f) \equiv W^{\hbar}(f)$ lead by Fourier transformation to the deformed --product for the periodic Weyl functions $W_{c}(f)$

$$
W_{c}(f){ }_{\hbar} W_{c}(g)=\exp \left\{-\frac{i}{2} \hbar \sigma(f, g)\right\} W_{c}(f+g), \quad W_{c}(f)^{*}=W_{c}(-f), \quad \forall f, g \in E .(
$$

In case of $\hbar=0$ equation (4.4) reduces to the commutative pointwise product of functions. We see that $\mathbb{F}$ is a non-surjective, but injective $*$-homomorphism from the commutative Banach*-algebra $\left(\mathcal{M}(E), \star_{0}\right)$ into the commutative $C^{*}$-algebra $\left(\mathrm{C}_{b}\left(E_{\tau}^{\prime}\right),{ }_{0}\right)$. Especially, $\left(\widehat{\mathcal{M}}(E)_{d f},{ }_{0}\right)$ resp. $\left(\widehat{\mathcal{M}}(E)_{d},{ }_{0}\right)$ are $\|\cdot\|_{0}$-dense sub-*-algebras of the $C^{*}$-algebra $\left(\operatorname{AP}\left(E_{\tau}^{\prime}\right),{ }_{0}\right)$ of the almost periodic functions. 


\subsection{Pre-symplectic geometry revisited}

We assume in the present Subsection a fixed locally convex topology $\tau$ on $E$, for which a $\tau$ continuous semi-norm $\varsigma$, satisfying (3.8), exists. Thus, in contradistinction to Subsection 2.5, $\sigma$ is now jointly $\tau$-continuous. We denote again the phase space manifold $E_{\tau}^{\prime}$ by $\mathrm{P}$. We modify, however, the notions of tangent and cotangent spaces, which is suggested from our $\tau$-continuous semi-norm $\varsigma$ on $E$. Let $f \mapsto[f]$ be the quotient map to form the quotient $E / \operatorname{ker}(\varsigma)$ with respect to the kernel $\operatorname{ker}(\varsigma):=\{f \in E \mid \varsigma(f)=0\}$. Then $\|[f]\|_{\varsigma}:=\varsigma(f)$ defines a norm on $E / \operatorname{ker}(\varsigma)$, the completion of which is denoted by $E_{\varsigma}$. Because of (3.8) the pre-symplectic form $\sigma$ extends $\|\cdot\|_{\varsigma}$-continuously to $E_{\varsigma}$ with the estimation

$$
\left|\sigma_{\varsigma}(\phi, \psi)\right| \leq c\|\phi\|_{\varsigma}\|\psi\|_{\varsigma}, \quad \forall \phi, \psi \in E_{\varsigma},
$$

where we have set $\sigma_{\varsigma}([f],[g]):=\sigma(f, g)$ for all $f, g \in E$.

Let $E_{\varsigma}^{\prime}$ consist of all $\mathbb{R}$-linear functionals $G: E \rightarrow \mathbb{R}$ for which there exists a constant $k \geq 0$ (depending on $G$ ) with $|G(f)| \leq k \varsigma(f) \forall f \in E$. By construction, $E_{\varsigma}^{\prime} \subseteq E_{\tau}^{\prime}$. Since $\operatorname{ker}(G) \supseteq \operatorname{ker}(\varsigma)$ for all $G \in E_{\varsigma}^{\prime}$ it follows that $E_{\varsigma}^{\prime}$ is the topological dual of $E_{\varsigma}$ with respect to the norm $\|\cdot\|_{\varsigma}$, identifying $G([f])$ with $G(f)$ for all $f \in E$.

In order to allow in

$$
d_{F} A(G):=\left.\frac{d A[c(t)]}{d t}\right|_{t=0}
$$

the differentiation only in the directions $G$ from $E_{\varsigma}^{\prime}$, we restrict at each $F \in \mathrm{P}$ the tangent space to

$$
{ }_{\varsigma} T_{F} \mathrm{P}:=E_{\varsigma}^{\prime} \subseteq E_{\tau}^{\prime}
$$

Considering on $E_{\varsigma}^{\prime}$ the $\sigma\left(E_{\varsigma}^{\prime}, E_{\varsigma}\right)$-topology, we obtain the cotangent space

$$
{ }_{\varsigma} T_{F}^{*} \mathrm{P}:=\left(E_{\varsigma}^{\prime}\right)^{\prime}=E_{\varsigma} \supseteq\{[f] \mid f \in E\} .
$$

In generalization of equation (2.15) we define a constant Poisson tensor field $\Sigma$ on the cotangent bundle ${ }_{\varsigma} T^{*} \mathrm{P}$ by $\Sigma_{F}:=-\sigma_{\varsigma}: E_{\varsigma} \times E_{\varsigma} \rightarrow \mathbb{R}$ for every $F \in \mathrm{P}$, which we extend complex bilinearly to the complexified cotangent bundle ${ }_{\varsigma}^{\mathbb{C}} T^{*} \mathrm{P}$. The associated Poisson bracket at the phase space point $F \in \mathrm{P}$ is canonically introduced as

$$
\begin{aligned}
\{A, B\}[F] & :=\Sigma_{F}\left(d_{F} A, d_{F} B\right) \\
& =-\sigma_{\varsigma}\left(d_{F} A_{1}, d_{F} B_{1}\right)-i \sigma_{\varsigma}\left(d_{F} A_{1}, d_{F} B_{2}\right)-i \sigma_{\varsigma}\left(d_{F} A_{2}, d_{F} B_{1}\right)+\sigma_{\varsigma}\left(d_{F} A_{2}, d_{F} B_{2}\right),
\end{aligned}
$$

where $A$ and $B$ are $\mathbb{C}$-valued functions on $\mathrm{P}$, which are differentiable in all directions $G \in E_{\varsigma}^{\prime}$ with $d_{F} A, d_{F} B \in{ }_{\varsigma}^{\mathbb{C}} T_{F}^{*} \mathrm{P}=E_{\varsigma}+i E_{\varsigma}$ for all $F \in \mathrm{P}$. The latter condition means for a function $A: \mathrm{P} \rightarrow \mathbb{C}$ that its total differential $\mathrm{P} \ni F \mapsto d_{F} A$ is continuous with respect to the $\sigma\left(E_{\varsigma}, E_{\varsigma}^{\prime}\right)$ topology. But this weak continuity does in general not ensure that $\mathrm{P} \ni F \mapsto\{A, B\}[F] \in \mathbb{C}$ is continuous. But, in virtue of (4.6), the Poisson bracket continuity is here automatically fulfilled, if $\mathrm{P} \ni F \mapsto d_{F} A$ and $\mathrm{P} \ni F \mapsto d_{F} B$ are strongly continuous, i.e., with respect to the norm $\|\cdot\|_{\varsigma}$ (and so in the specified weak*-topology on $\mathrm{P}$ ).

For the Weyl functions $W_{c}(f): \mathrm{P} \rightarrow \mathbb{C}$ from equation (2.13) we obtain with definition (4.7) $d_{F} W_{c}(f)(G)=i G(f) W_{c}(f)[F]$. If we restrict differentiation to the directions from $E_{\varsigma}^{\prime}$, then the identification $G([f]) \equiv G(f)$ leads to

$$
d_{F} W_{c}(f)=i \exp \{i F(f)\}[f]=i W_{c}(f)[F][f] \in{ }_{\varsigma}^{\mathbb{C}} T_{F}^{*} \mathrm{P}=E_{\varsigma}^{\prime}+i E_{\varsigma}^{\prime}, \quad \forall F \in \mathrm{P},
$$


which deviates from equation (2.16). (Note that $E_{\varsigma}^{\prime}$ separates points not only on $E_{\varsigma}$, but also on $E$, if and only if $\varsigma$ is a proper norm and not only a semi-norm). Inserting into equation (4.8) and noting $\sigma_{\varsigma}([f],[g])=\sigma(f, g)$ yields

$$
\left\{W_{c}(f), W_{c}(g)\right\}=\sigma(f, g) W_{c}(f+g)=\sigma_{\varsigma}([f],[g]) W_{c}(f+g), \quad \forall f, g \in E,
$$

which coincides with the former Poisson bracket formula of equation (2.17).

If the Poisson bracket $\{\cdot, \cdot\}$ of (4.8) would commute with the Fourier integration $\widehat{\mu}=$ $\int_{E} d \mu(f) W_{c}(f)$, then we would obtain from (4.10)

$$
\begin{aligned}
\{\widehat{\mu}, \widehat{\nu}\}[F] & =\int_{E} d \mu(f) \int_{E} d \nu(g)\left\{W_{c}(f), W_{c}(g)\right\}[F] \\
& =\int_{E} d \mu(f) \int_{E} d \nu(g) \sigma(f, g) \exp \{i F(f+g)\}=\mathbb{F}\{\mu, \nu\}_{0}[F],
\end{aligned}
$$

where $\{\cdot, \cdot\}_{0}$ is the Poisson bracket for the measures from equation (3.9). This suggests to take as Poisson algebras the Fourier transforms of the spaces $\mathcal{P}$ from Theorem 3.

Theorem 5. Let $n \in \mathbb{N} \bigcup\{\infty\}$. Then $\widehat{\mu} \in \widehat{\mathcal{M}}_{\varsigma}^{n}\left(E_{\tau}^{\prime}\right)$ is n-times continuously differentiable in all the directions of $E_{\varsigma}^{\prime}$, and moreover, for each $F \in \mathrm{P} \equiv E_{\tau}^{\prime}$ its total differential $d_{F} \widehat{\mu} \in{ }_{\varsigma}^{\mathbb{T}} T_{F}^{*} \mathrm{P}$ is given by the Bochner integral

$$
d_{F} \widehat{\mu}=i \int_{E} d \mu(f) \exp \{i F(f)\}[f]=\int_{E} d \mu(f) d_{F} W_{c}(f),
$$

which converges with respect to the norm $\|\cdot\|_{\varsigma}$ on the Banach space ${ }_{\varsigma}^{\mathbb{C}} T_{F}^{*} \mathrm{P}=E_{\varsigma}+i E_{\varsigma}$.

The Fourier transformation $\mathbb{F}$ is a Poisson automorphism transforming the measure Poisson bracket $\{\cdot, \cdot\}_{0}$ from equation (3.9) into the function Poisson bracket $\{\cdot, \cdot\}$ from equation (4.8), that is,

$$
\{\widehat{\mu}, \widehat{\nu}\}=\mathbb{F}\{\mu, \nu\}_{0}, \quad \forall \mu, \nu \in \mathcal{M}_{\varsigma}^{1}(E) .
$$

Let $\widehat{\mathcal{P}}:=\mathbb{F} \mathcal{P}$, with the choices for $\mathcal{P}$ as in Theorem 3 . Then $\left(\widehat{\mathcal{P}},{ }_{0},\{\cdot, \cdot\}\right)$ constitutes a Poisson algebra contained in $\widehat{\mathcal{M}}_{\varsigma}^{\infty}\left(E_{\tau}^{\prime}\right)$, which in addition is invariant under all deformed products $\hbar$, $\hbar \in \mathbb{R}$. Especially $\left(\widehat{\mathcal{M}}_{\varsigma}^{\infty}\left(E_{\tau}^{\prime}\right),{ }_{0},\{\cdot, \cdot\}\right)$ is a Poisson algebra of this type.

Proof. For $\mu \in \mathcal{M}_{\varsigma}^{n}(E)$ and $G \in E_{\varsigma}^{\prime}$ let us define the measure $\mu_{G}$ by $d \mu_{G}(f)=G(f) d \mu(f)$. Since $|G(f)|=|G([f])| \leq\|G\|_{\varsigma}\|[f]\|_{\varsigma}=\|G\|_{\varsigma} \varsigma(f) \forall f \in E$, we conclude that $\left|\mu_{G}\right| \leq\|G\|_{\varsigma}|\mu|_{\varsigma}^{1}$, and hence $\mu_{G} \in \mathcal{M}_{\varsigma}^{n-1}(E)$. Consequently, we may differentiate according to equation (4.7) as

$$
\begin{aligned}
d_{F} \widehat{\mu}(G) & =\left.\frac{d \widehat{\mu}[F+t G]}{d t}\right|_{t=0}=\left.\frac{d}{d t}\left(\int_{E} d \mu(f) \exp \{i F(f)+i t G(f)\}\right)\right|_{t=0} \\
& =i \int_{E} d \mu(f) G(f) \exp \{i F(f)\}=\widehat{\mu_{G}}[F]
\end{aligned}
$$

implying $\mathrm{P} \ni F \mapsto d_{F} \widehat{\mu}(G)$ to be an element of $\widehat{\mathcal{M}}_{\varsigma}^{n-1}(\mathrm{P})$, especially being continuous. Iteration shows that $\widehat{\mu}$ is $n$-times continuously differentiable in all the directions of $E_{\varsigma}^{\prime}$. Now $G([f])=G(f)$ yields (4.12). Note, for the Bochner integral it is necessary that the range of the weak measurable quotient map $\operatorname{supp}(\mu) \ni f \mapsto[f]$ has to be separable [40, p. 350], which is ensured because $\mu$ is approximable by a sequence of measures on finite dimensional subspaces of $E$. Inserting (4.12) into the Poisson bracket from equation (4.8) makes our consideration in equation (4.11) rigorous, and thus yields $\{\widehat{\mu}, \widehat{\nu}\}=\mathbb{F}\{\mu, \nu\}_{0}$. The assertions concerning the Poisson algebras $\widehat{\mathcal{P}}$ are immediate.

Especially, with the identification of $W^{\hbar}(f) \equiv \delta(f)$ of Proposition 2, the Poisson isomorphism in Corollary 1 agrees just with the Fourier transformation. This case is covered by the measure Poisson algebra (IV) of Theorem 3. 


\section{Representations, folia, and enveloping $C^{*}$-algebras}

The present Section makes representation theory available for the generalized Weyl quantization in Section 6. Recall that we assumed our pre-symplectic space $(E, \sigma)$ infinite dimensional.

\subsection{Folia and quasi-equivalence classes of representations}

Let us sketch the connection between folia and representations, as it is treated e.g. in [47, 48, 44, 49]; an overview is given in [50].

A folium $\mathcal{F}$ of a $C^{*}$-algebra $\mathcal{A}$ is a specific face of the state space $\mathcal{S}(\mathcal{A})$ : it is a norm-closed, convex subset of $\mathcal{S}(\mathcal{A})$, which is invariant under so-called small perturbations, meaning that $\omega \in \mathcal{F}$ implies $\omega_{B} \in \mathcal{F}$ for all $B \in \mathcal{A}$ with $\left\langle\omega ; B^{*} B\right\rangle \neq 0$. Here $\omega_{B}$ is the state on $\mathcal{A}$ given by $\left\langle\omega_{B} ; \cdot\right\rangle=\left\langle\omega ; B^{*} \cdot B\right\rangle\left\langle\omega ; B^{*} B\right\rangle^{-1}$. The collection of all folia of $\mathcal{A}$ is denoted by $\operatorname{fol}(\mathcal{A})$.

Two non-degenerate representations $\Pi_{1}$ and $\Pi_{2}$ of the $C^{*}$-algebra $\mathcal{A}$ are quasi-equivalent (see e.g. [51, Section 2.4.4]), if and only if there exists a $*$-isomorphism $\alpha$ from the von Neumann algebra ${\overline{\Pi_{1}(\mathcal{A})}}^{w}$ onto the von Neumann algebra ${\overline{\Pi_{2}(\mathcal{A})}}^{w}$ with $\alpha\left(\Pi_{1}(A)\right)=\Pi_{2}(A), \forall A \in \mathcal{A}$. The upper index " $w$ " indicates closure with respect to the $\sigma$-weak operator topology of the representation Hilbert space. We denote by $\operatorname{rep}(\mathcal{A})$ the set of all quasi-equivalence classes of

representations. So, writing subsequently $\Pi \in \operatorname{rep}(\mathcal{A})$ we denote a quasi-equivalence class of representations, and $\overline{\Pi(\mathcal{A})}^{w}$ indicates the associated $\mathrm{W} *$-algebra, which is abstracted from the *-isomorphic von Neumann algebras given by the representations in the quasi-equivalence class.

There is a one-to-one correspondence $\mathcal{F} \mapsto \Pi_{\mathcal{F}}$ from $\operatorname{fol}(\mathcal{A})$ onto $\operatorname{rep}(\mathcal{A})$, which preserves the partial order relations

$$
\mathcal{F}_{1} \subseteq \mathcal{F}_{2} \subseteq \mathcal{S}(\mathcal{A}) \quad \mapsto \quad \Pi_{\mathcal{F}_{1}} \leq \Pi_{\mathcal{F}_{2}} \leq \Pi_{u}, \quad \mathcal{F}_{1}, \mathcal{F}_{2} \in \operatorname{fol}(\mathcal{A})
$$

The partial ordering in the family of folia $\operatorname{fol}(\mathcal{A})$ is the set inclusion, and " $\leq$ " means the partial ordering of being a subrepresentation, up to quasi-equivalence. The quasi-equivalence class $\Pi_{\mathcal{F}} \in \operatorname{rep}(\mathcal{A})$, corresponding to a given $\mathcal{F} \in \operatorname{fol}(\mathcal{A})$, may be constructively determined by means of the special representative obtained as the direct sum of GNS representations over all states in $\mathcal{F}$.

$\Pi_{u}$ denotes the universal representation of the $C^{*}$-algebra. For $\Pi_{\mathcal{F}_{1}} \leq \Pi_{\mathcal{F}_{2}}$ there exists a central projection $P \in{\overline{\Pi_{\mathcal{F}_{2}}(\mathcal{A})}}^{w}$ such that ${\overline{\overline{\mathcal{F}}_{1}(\mathcal{A})}}^{w}={\overline{\Pi_{\mathcal{F}_{2}}(\mathcal{A})}}^{w} P$, and we sometimes identify $\Pi_{\mathcal{F}_{1}}(A)$ with $\Pi_{\mathcal{F}_{1}}(A) P$, for $A \in \mathcal{A}$.

In the mentioned correspondence, the smallest folium $\mathcal{F}_{\omega}$ containing the state $\omega$ corresponds to the equivalence class of its GNS representation $\Pi_{\omega}$, that is, $\Pi_{\omega}$ is a representative of the class

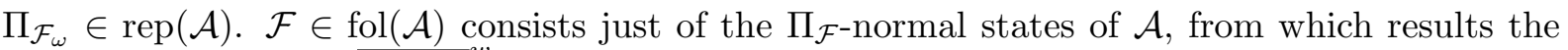
Banach space duality ${\overline{\Pi_{\mathcal{F}}(\mathcal{A})}}^{w}=\mathrm{LH}(\mathcal{F})^{*}$. Generally we do not distinguish notationally between $\omega \in \mathcal{F}$ as a state on $\mathcal{A}$ and its unique normal extension $\omega$ to the $\mathrm{W} *$-algebra ${\overline{\Pi_{\mathcal{F}}(\mathcal{A})}}^{w}$ (writing $\langle\omega ; A\rangle$ for $A \in \mathcal{A}$ as well as for $A \in{\overline{\Pi_{\mathcal{F}}(\mathcal{A})}}^{w}$ ).

If $\mathcal{A}=C^{*}(\mathcal{B})$ is the enveloping $C^{*}$-algebra of a Banach-*-algebra $\mathcal{B}$ (see e.g. [43]), then $\mathcal{A}$ and $\mathcal{B}$ have the same representations given by continuous extensions resp. restrictions, in which case we sometimes use the notation $\operatorname{fol}(\mathcal{B}):=\operatorname{fol}(\mathcal{A})$ and $\operatorname{rep}(\mathcal{B}):=\operatorname{rep}(\mathcal{A})$. Throughout the present work we do not distinguish notationally between representations of $\mathcal{B}$ and their unique continuous extension to the enveloping $C^{*}$-algebra $\mathcal{A}$.

\section{$5.2 \tau$-continuous representations of the Weyl algebra}

We denote by $\mathcal{T}(E, \sigma)$ the set of all topologies $\tau$ on $E$ such that for each $f \in E$ the maps $E \ni g \mapsto f+g \in E$ and $E \ni g \mapsto \sigma(f, g) \in \mathbb{R}$ are $\tau$-continuous, what means just the separate $\tau$-continuity of the addition and of the antisymmetric form $\sigma$. The set $\mathcal{T}(E, \sigma)$ of topologies is 
directed with respect to the natural partial ordering "finer than". So, each vector space topology on $E$, for which $\sigma$ is separately continuous, is an element of $\mathcal{T}(E, \sigma)$.

Let $\tau \in \mathcal{T}(E, \sigma)$. A state $\omega \in \mathcal{S}(\mathcal{W}(E, \hbar \sigma))$ is called $\tau$-continuous, if its characteristic function

$$
E \ni f \longmapsto\left\langle\omega ; W^{\hbar}(f)\right\rangle=: C_{\omega}(f)
$$

is $\tau$-continuous. A representation $\Pi_{\hbar}$ of $\mathcal{W}(E, \hbar \sigma)$ is denoted $\tau$-continuous, if $E \ni f \mapsto$ $\Pi_{\hbar}\left(W^{\hbar}(f)\right)$ is continuous with respect to the topology $\tau$ on $E$ and to any weak operator topology on the image space. Recall that all of the weak operator topologies (weak, strong, $\sigma$-strong*, ...) are equivalent on the group of unitary operators. Clearly $\tau$-continuity carries over to the whole quasi-equivalence class $\Pi_{\hbar} \in \operatorname{rep}(\mathcal{W}(E, \hbar \sigma)$ ), and hence the mapping (see equation (2.8))

$$
E \ni f \longmapsto \Pi_{\hbar}\left(W^{\hbar}(f)\right)=\pi_{\hbar}(f) \in{\overline{\Pi_{\hbar}(\mathcal{W}(E, \hbar \sigma))}}^{w}
$$

is continuous with respect to $\tau$ on $E$ and to the $\sigma$-strong (equivalently $\sigma$-weak) topology on the $\mathrm{W} *$-algebra ${\overline{\Pi_{\hbar}(\mathcal{W}(E, \hbar \sigma))}}^{w}$. If for $\tau$ the addition is jointly continuous, then we may regard $E$ as a topological group, and $\pi_{\hbar}$ may be viewed as a quasi-equivalence class of $\tau$-continuous, projective, unitary group representations.

Let us say a word about the existence of $\tau$-continuous states, and, via GNS construction, about the existence of $\tau$-continuous representations. By $[31,52]$ the characteristic functions $(5.2)$ of the states on $\mathcal{W}(E, \hbar \sigma)$ coincide with the convex set $\mathcal{C}(E, \hbar \sigma)$ of functions $C: E \rightarrow \mathbb{C}$, which are projective positive-definite (i.e. $\sum_{i, j=1}^{n} \overline{z_{i}} z_{j} \exp \left\{\frac{i}{2} \hbar \sigma\left(f_{i}, f_{j}\right)\right\} C\left(f_{j}-f_{i}\right) \geq 0$ for arbitrary $z_{j} \in \mathbb{C}$, $\left.f_{j} \in E, n \in \mathbb{N}\right)$, and are normalized (i.e. $\left.C(0)=1\right) \cdot \mathcal{C}(E, \hbar \sigma)$ is always non-empty, but a priori it is not known, if it contains elements which are $\tau$-continuous. Let us first consider the case $\sigma \neq 0$ and $\hbar \neq 0$. It is well known that a Gaussian function $E \ni f \mapsto \exp \left\{-\frac{1}{4} s(f, f)\right\}$ ( $s$ is a positive symmetric $\mathbb{R}$-bilinear form on $E$ ) is an element of $\mathcal{C}(E, \hbar \sigma)$, if and only if $\hbar^{2} \sigma(f, g)^{2} \leq s(f, f) s(g, g)$ for all $f, g \in E$. So, provided there exists such an jointly $\tau$-continuous form $s$, there exists a $\tau$-continuous state. For finite dimensional $E$ such forms $s$ are easily constructed. However, for infinite dimensions there may exist pathological (pre-) symplectic spaces $(E, \sigma)$, for which no $\tau$-continuous form $s$, or even worse, no $\tau$-continuous characteristic function exists at all, excluding $\tau$-continuous states. For such "symplectic pathology" we refer to [53]. For the standard example of a symplectic space (see beginning of Subsection 2.2) one may take, however, the $\|\cdot\|$-continuous form $s(f, g)=|\hbar| \Re(f \mid g)$ in order to show the existence of $\tau$-continuous states for every $\tau$ finer than this norm.

Hence for our subsequent investigations, we may assume a pre-symplectic space $(E, \sigma)$ for which $\mathcal{W}(E, \hbar \sigma)$ has $\tau$-continuous states for many $\tau \in \mathcal{T}(E, \sigma)$. (This carries through to the quotient $E / \operatorname{ker}_{\sigma}$, which is needed in the proof of Lemma 8.)

For $\hbar=0$, where $\mathcal{C}(E, 0)$ consists of all positive-definite, normalized functions, the existence question of $\tau$-continuous states is answered easily. Every character on the vector group $E$ is an element of $\mathcal{C}(E, 0)$ and may be interpreted as the characteristic function of a unique state on the commutative Weyl algebra $\mathcal{W}(E, 0)$. Consequently, if $\tau$ is an arbitrary locally convex topology on $E$, then each $\tau$-continuous character $E \ni f \mapsto \exp \{i F(f)\}$, where $F \in E_{\tau}^{\prime}$, defines a $\tau$-continuous state.

It is shown in $[54,50,55]$ that for each topology $\tau \in \mathcal{T}(E, \sigma)$ the set of states

$$
\mathcal{F}_{\hbar}^{\tau}=\{\omega \in \mathcal{S}(\mathcal{W}(E, \hbar \sigma)) \mid \omega \text { is } \tau \text {-continuous }\}
$$

constitutes a folium in the state space $\mathcal{S}(\mathcal{W}(E, \hbar \sigma))$. The representation $\Pi_{\hbar}^{\tau} \in \operatorname{rep}(\mathcal{W}(E, \hbar \sigma))$, associated with $\mathcal{F}_{\hbar}^{\tau}$, is the largest $\tau$-continuous representation of the Weyl algebra $\mathcal{W}(E, \hbar \sigma)$, 
that is, each $\tau$-continuous $\Pi_{\hbar} \in \operatorname{rep}(\mathcal{W}(E, \hbar \sigma))$ is a subrepresentation of $\Pi_{\hbar}^{\tau}$. By Lemma 8 of the Appendix, $\Pi_{\hbar}^{\tau}$ is faithful.

The partial ordering on $\mathcal{T}(E, \sigma)$ carries over to the associated folia of $\mathcal{W}(E, \hbar \sigma)$

$$
\tau_{1} \leq \tau_{2} \leq d \quad \Longrightarrow \quad \mathcal{F}_{\hbar}^{\tau_{1}} \subseteq \mathcal{F}_{\hbar}^{\tau_{2}} \subseteq \mathcal{F}_{\hbar}^{d}=\mathcal{S}(\mathcal{W}(E, \hbar \sigma))
$$

( $d$ indicates the discrete topology), which leads with equation (5.1) to the partial ordering of the associated (quasi-equivalence classes of) representations according to their continuity properties.

\subsection{Folium of regular states of the Weyl algebra}

A representation $\Pi_{\hbar} \in \operatorname{rep}(\mathcal{W}(E, \hbar \sigma))$ is called regular, if for each $f \in E$ the mapping $\mathbb{R} \ni$ $t \mapsto \Pi_{\hbar}\left(W^{\hbar}(t f)\right)$ is $\sigma$-strongly continuous in a neighbourhood of the origin, or equivalently, if $E_{\alpha} \ni f \mapsto \Pi_{\hbar}\left(W^{\hbar}(f)\right)$ is $\sigma$-strongly continuous on every finite dimensional subspace $E_{\alpha}$ of $E$ with respect to the unique vector space topology on $E_{\alpha}$. A state $\omega$ on $\mathcal{W}(E, \hbar \sigma)$ is termed regular, if $\mathbb{R} \ni t \mapsto\left\langle\omega ; W^{\hbar}(t f)\right\rangle$ is continuous in a neighbourhood of the origin for each $f \in E$. This is equivalent to its GNS representation being regular [2, Section 5.2.3]. The set of regular states

$$
\mathcal{F}_{\hbar}^{\mathrm{reg}}:=\{\omega \in \mathcal{S}(\mathcal{W}(E, \hbar \sigma)) \mid \omega \text { is regular }\}
$$

again constitutes a folium of $\mathcal{W}(E, \hbar \sigma)$. The representation associated with $\mathcal{F}_{\hbar}^{\text {reg }}$, denoted by $\Pi_{\hbar}^{\text {reg }} \in \operatorname{rep}(\mathcal{W}(E, \hbar \sigma))$, is the largest regular representation of $\mathcal{W}(E, \hbar \sigma)$.

Regularity suggests that $E$ carries a vector space topology which is induced by its finite dimensional subspaces, namely the finest locally convex topology $\tau_{\text {il }}$ (introduced at the beginning of Section 3), which already has been discussed in [56]. But for every locally convex topology on $E$ its restriction to $E_{\alpha}$ just gives the natural unique vector space topology on $E_{\alpha}$.

Since every linear map from $E$ into a locally convex space is continuous with respect to $\tau_{\text {il }}$, it follows that the pre-symplectic form $\sigma$ is at least separately $\tau_{\mathrm{il}}$-continuous, and consequently, $\tau_{\text {il }} \in \mathcal{T}(E, \sigma)$, what demonstrates the existence of a locally convex element in $\mathcal{T}(E, \sigma)$. In general, $\sigma$ may not be jointly $\tau_{\mathrm{il}}$-continuous on $E \times E$, but only bounded and hypocontinuous [57]. In any case, the standard example of a symplectic space, $\sigma=\Im(\cdot \mid \cdot)$, is jointly $\tau_{\text {il }}$-continuous (since $\tau_{\text {il }}$ is finer than the norm topology arising from $\left.(\cdot \mid \cdot)\right)$.

The folium of all $\tau_{\mathrm{il}}$-continuous states on $\mathcal{W}(E, \hbar \sigma)$ is denoted by $\mathcal{F}_{\hbar}^{\text {il }}$, and the associated largest $\tau_{\mathrm{il}}$-continuous representation by $\Pi_{\hbar}^{\mathrm{il}} \in \operatorname{rep}(\mathcal{W}(E, \hbar \sigma))$. Since $\tau_{\mathrm{il}}$ is the finest locally convex topology in $\mathcal{T}(E, \sigma)$ we know the ordering relations

$$
\mathcal{F}_{\hbar}^{\tau} \subseteq \mathcal{F}_{\hbar}^{\mathrm{il}} \subseteq \mathcal{F}_{\hbar}^{\mathrm{reg}} \quad \Longleftrightarrow \quad \Pi_{\hbar}^{\tau} \leq \Pi_{\hbar}^{\mathrm{il}} \leq \Pi_{\hbar}^{\mathrm{reg}},
$$

for each locally convex $\tau \in \mathcal{T}(E, \sigma)$. Because the characteristic functions (5.2) are non-linear, it is for infinite dimensional $E$ not possible to show that regularity implies $\tau_{\text {il }}$-continuity. In general one knows that $\mathcal{F}_{\hbar}^{\text {il }}$ is a proper subfolium of $\mathcal{F}_{\hbar}^{\text {reg }}$. In [57] an example of a test function space $(E, \sigma)$ is given, for which the two folia coincide.

For infinite dimensional $E$ there exists a continuum of inequivalent regular representations of $\mathcal{W}(E, \hbar \sigma)$ and, for given locally convex $\tau$, one has even a continuum of inequivalent $\tau$ continuous representations.

\subsection{Representations of the measure Banach-*-algebras}

We fix here an $\hbar \in \mathbb{R}$ and consider a certain type of Hilbert space representations of the Banach-*-algebra $\left(\mathcal{M}(E), \star_{\hbar}\right)$. First, however, we restrict ourselves to $\left(\mathcal{M}\left(E_{\alpha}\right), \star_{\hbar}\right)$ for the finite dimensional, and thus locally compact, subspaces $E_{\alpha}$ of $E$, referring some facts known from the literature (e.g. [32, 44, 46]). 
There exists a 1:1:1:1-correspondence between the $\sigma$-strongly continuous unitary representations $\left(\pi_{\hbar}, \mathcal{H}_{\hbar}\right)$ of the additive group $E_{\alpha}$, the non-degenerate regular (=continuous) representations $\left(\Pi_{\hbar}, \mathcal{H}_{\hbar}\right)$ of $\left(\mathcal{M}\left(E_{\alpha}\right)_{d}, \star_{\hbar}\right)$ resp. of $\mathcal{W}\left(E_{\alpha}, \hbar \sigma\right)$, the non-degenerate representations $\left(\Pi_{\hbar}, \mathcal{H}_{\hbar}\right)$ of $\left(\mathcal{M}\left(E_{\alpha}\right)_{a}, \star_{\hbar}\right)$, and those representations $\left(\Pi_{\hbar}, \mathcal{H}_{\hbar}\right)$ of $\left(\mathcal{M}\left(E_{\alpha}\right), \star_{\hbar}\right)$ for which $\Pi_{\hbar}\left(\mathcal{M}\left(E_{\alpha}\right)_{a}\right) \mathcal{H}_{\hbar}$ is dense in the representation Hilbert space $\mathcal{H}_{\hbar}$. This correspondence is given by $\Pi_{\hbar}(\mu)=$ $\int_{E_{\alpha}} d \mu(f) \pi_{\hbar}(f), \mu \in \mathcal{M}\left(E_{\alpha}\right)$. It preserves irreducibility. Such a $\left(\Pi_{\hbar}, \mathcal{H}_{\hbar}\right)$ is called regular in the literature, since it extends the original regular representation of $\left(\mathcal{M}\left(E_{\alpha}\right)_{d}, \star_{\hbar}\right)$ resp. of $\mathcal{W}\left(E_{\alpha}, \hbar \sigma\right)$ to a representation of the larger algebra $\left(\mathcal{M}\left(E_{\alpha}\right), \star_{\hbar}\right)$ resp. $C^{*}\left(\mathcal{M}\left(E_{\alpha}\right), \star_{\hbar}\right)$.

Since a regular representation $\left(\Pi_{\hbar}, \mathcal{H}_{\hbar}\right)$ of $\left(\mathcal{M}\left(E_{\alpha}\right), \star_{\hbar}\right)$ acts non-degenerately on the closed *-ideal of the absolutely continuous measures $\mathcal{M}\left(E_{\alpha}\right)_{a}$, one has

$$
\left\|\Pi_{\hbar}(\mu)\right\|=\sup \left\{\left\|\Pi_{\hbar}\left(\mu \star_{\hbar} \gamma\right)\right\| \mid \gamma \in \mathcal{M}\left(E_{\alpha}\right)_{a},\|\gamma\|_{1} \leq 1\right\}=\lim _{n \rightarrow \infty}\left\|\Pi_{\hbar}\left(\mu \star_{\hbar} \gamma_{n}\right)\right\|,
$$

where $\left(\gamma_{n}\right)_{n \in \mathbb{N}}$, with $\left\|\gamma_{n}\right\|_{1}=1$, is an approximate identity for $\left(\mathcal{M}\left(E_{\alpha}\right)_{a}, \star_{\hbar}\right)$ (because $\Pi_{\hbar}(\nu) \xi=$ $\lim _{n} \Pi_{\hbar}\left(\gamma_{n}\right) \Pi_{\hbar}(\nu) \xi$ for $\nu \in \mathcal{M}\left(E_{\alpha}\right)_{a}$ and $\xi \in \mathcal{H}_{\Pi}$, and $\Pi_{\hbar}\left(\mathcal{M}\left(E_{\alpha}\right)_{a}\right) \mathcal{H}_{\Pi}$ is dense in $\mathcal{H}_{\Pi}$, where $\left.\left\|\Pi_{\hbar}(\mu)\right\|=\sup _{\|\eta\|=1}\left\|\Pi_{\hbar}(\mu) \eta\right\|\right)$. For $\mathcal{M}\left(E_{\alpha}\right)_{a}$ there exist countable approximate identities (since $E_{\alpha}$ is finite dimensional), as e.g. such $\gamma_{n} \in \mathcal{M}\left(E_{\alpha}\right)_{a}^{+}$which are concentrated in the ball of radius $n^{-1}$ around the origin.

For an arbitrary representation $\left(\Psi_{\hbar}, \mathcal{H}_{\hbar}\right)$ of $\left(\mathcal{M}\left(E_{\alpha}\right), \star_{\hbar}\right)$ one may separate out a regular part: $\mathcal{M}\left(E_{\alpha}\right)_{a}$ being a closed $*$-ideal ensures that the orthogonal projection $P$ onto the closure of $\Psi_{\hbar}\left(\mathcal{M}\left(E_{\alpha}\right)_{a}\right) \mathcal{H}_{\hbar}$ commutes with each $\Psi_{\hbar}(\mu), \mu \in \mathcal{M}\left(E_{\alpha}\right)$. Hence $\left(\Psi_{\hbar}, \mathcal{H}_{\hbar}\right)$ decomposes uniquely into the direct sum $\Psi_{\hbar}=\Pi_{\hbar} \oplus \Pi_{\hbar}^{N}$, where $\Pi_{\hbar}:=P \Psi_{\hbar}$ is an regular representation of $\left(\mathcal{M}\left(E_{\alpha}\right), \star_{\hbar}\right)$, and where $\Pi_{\hbar}^{N}:=(\mathbb{1}-P) \Psi_{\hbar}$ is a representation of $\left(\mathcal{M}\left(E_{\alpha}\right), \star_{\hbar}\right)$ which vanishes on the *ideal $\mathcal{M}\left(E_{\alpha}\right)_{a}$, which we simply call a non-regular representation.

The Banach-*-algebra $\left(\mathcal{M}\left(E_{\alpha}\right), \star_{\hbar}\right)$ is a dense sub-*-algebra of its enveloping $C^{*}$-algebra $C^{*}\left(\mathcal{M}\left(E_{\alpha}\right), \star_{\hbar}\right)$, the $C^{*}$-norm of the latter being written as $\|\cdot\|_{\hbar}$. One finds that $C^{*}\left(\mathcal{M}\left(E_{\alpha}\right)_{d}, \star_{\hbar}\right)$ $=\mathcal{W}\left(E_{\alpha}, \hbar \sigma\right)$ is a sub- $C^{*}$-algebra, and both $C^{*}\left(\mathcal{M}\left(E_{\alpha}\right)_{a}, \star_{\hbar}\right)$ and $C^{*}\left(\mathcal{M}\left(E_{\alpha}\right)_{c}, \star_{\hbar}\right)$ are closed *ideals of $C^{*}\left(\mathcal{M}\left(E_{\alpha}\right), \star_{\hbar}\right)$. The algebras $\left(\mathcal{M}\left(E_{\alpha}\right)_{a}, \star_{\hbar}\right)$ and $C^{*}\left(\mathcal{M}\left(E_{\alpha}\right)_{a}, \star_{\hbar}\right)$ are denoted twisted group Banach-*-algebra resp. twisted group $C^{*}$-algebra of the vector group $E_{\alpha}$ with respect to the multiplier (2.5).

The $L^{2}$-representation $\Pi_{\hbar}^{L^{2}}$, given by $\Pi_{\hbar}^{L^{2}}(\mu) \phi:=\mu \star \hbar \phi$ for $\phi \in \mathrm{L}^{2}\left(E_{\alpha}\right)$, is an example of an injective regular representation of $\left(\mathcal{M}\left(E_{\alpha}\right), \star_{\hbar}\right)$ (e.g. [32], [44]). The operator-norm-closure of $\Pi_{\hbar}^{L^{2}}\left(\mathcal{M}\left(E_{\alpha}\right)_{a}\right)$ is called restricted twisted group $C^{*}$-algebra. If $\sigma$ is non-degenerate on $E_{\alpha}$ and $\hbar \neq 0$, then it follows that these two twisted group $C^{*}$-algebras coincide (by Lemma 6(a) below), and are $*$-isomorphic to the $C^{*}$-algebra of compact operators on a separable Hilbert space [58, 59], cf. also [46].

$C^{*}\left(\mathcal{M}\left(E_{\alpha}\right)_{a}, \star_{\hbar}\right)$ being a closed $*$-ideal of $C^{*}\left(\mathcal{M}\left(E_{\alpha}\right), \star_{\hbar}\right)$ ensures the existence of a canonical *-homomorphism $m_{\hbar}$ from $C^{*}\left(\mathcal{M}\left(E_{\alpha}\right), \star_{\hbar}\right)$ into the multiplier $C^{*}$-algebra $\mathbb{M}_{\hbar}^{\alpha}$ of $C^{*}\left(\mathcal{M}\left(E_{\alpha}\right)_{a}, \star_{\hbar}\right)$ (e.g. [60, VIII.1], [61, III.6]). The above 1:1:1:1-correspondence shows that the extension of the largest regular representation $\Pi_{\hbar}^{\text {reg }}$ of $\mathcal{W}\left(E_{\alpha}, \hbar \sigma\right)$ resp. of $\left(\mathcal{M}\left(E_{\alpha}\right)_{d}, \star_{\hbar}\right)$ leads just to the universal representation of the twisted group $C^{*}$-algebra $C^{*}\left(\mathcal{M}\left(E_{\alpha}\right)_{a}, \star_{\hbar}\right)$. Hence $\mathbb{M}_{\hbar}^{\alpha}$ may be realized as a sub- $C^{*}$-algebra of the $\mathrm{W} *$-algebra ${\overline{\Pi_{\hbar}^{\text {reg }}\left(\mathcal{M}\left(E_{\alpha}\right)_{a}\right)}}^{w}$, in which case $m_{\hbar}$ coincides with $\Pi_{\hbar}^{\text {reg }}$. By the subsequent Lemma (also by Lemma 8 of the Appendix) $\Pi_{\hbar}^{\text {reg }}$ in addition acts faithfully on the discrete twisted group algebra $C^{*}\left(\mathcal{M}\left(E_{\alpha}\right)_{d}, \star_{\hbar}\right)=\mathcal{W}\left(E_{\alpha}, \hbar \sigma\right)$. Consequently, also $m_{\hbar}$ acts faithfully on the sub- $C^{*}$-algebra $C^{*}\left(\mathcal{M}\left(E_{\alpha}\right)_{d}, \star_{\hbar}\right)$, and the latter may be considered as sub- $C^{*}$ algebra of $\mathbb{M}_{\hbar}^{\alpha}$, too.

Lemma 5. Let $\Pi_{\hbar}$ be a regular representation of $\left(\mathcal{M}\left(E_{\alpha}\right)\right.$, $\left.\star_{\hbar}\right)$. If the representation $\Pi_{\hbar}$ acts faithfully on $C^{*}\left(\mathcal{M}\left(E_{\alpha}\right)_{a}, \star_{\hbar}\right)$, then it is faithful on $C^{*}\left(\mathcal{M}\left(E_{\alpha}\right)_{d}, \star_{\hbar}\right)=\mathcal{W}\left(E_{\alpha}, \hbar \sigma\right)$, too. 
Proof. $C^{*}\left(\mathcal{M}\left(E_{\alpha}\right)_{a}, \star_{\hbar}\right)$ is a thick $*$-ideal in its multiplier algebra $\mathbb{M}_{\hbar}^{\alpha}$. So every faithful representation of $C^{*}\left(\mathcal{M}\left(E_{\alpha}\right)_{a}, \star_{\hbar}\right)$ extends uniquely to a faithful representation of $\mathbb{M}_{\hbar}^{\alpha}$ (e.g. [61, Proposition III.6.25] and its proof). Now restrict to $C^{*}\left(\mathcal{M}\left(E_{\alpha}\right)_{d}, \star_{\hbar}\right)$.

Because of the above decomposition theorem of representations $\Psi_{\hbar}=\Pi_{\hbar} \oplus \Pi_{\hbar}^{N}, m_{\hbar}$ possibly may not act faithfully on the $C^{*}$-algebra $C^{*}\left(\mathcal{M}\left(E_{\alpha}\right), \star_{\hbar}\right)$, nevertheless $m_{\hbar}$ acts injectively on the measure space $\mathcal{M}\left(E_{\alpha}\right)$ itself $\left(\mu \star_{\hbar} \nu=0\right.$ (resp. $\nu \star_{\hbar} \mu=0$ ) for all $\nu \in \mathcal{M}\left(E_{\alpha}\right)_{a}$ yields $\mu=0$ ). In Subsection 6.3 we show the lower semicontinuity of $\mathbb{R} \ni \hbar \mapsto\left\|m_{\hbar}(\mu)\right\|=\left\|\Pi_{\hbar}^{\mathrm{reg}}(\mu)\right\|$ for all $\mu \in \mathcal{M}\left(E_{\alpha}\right)$, but continuity follows only for those measures $\mu$ satisfying $\|\mu\|_{\hbar}=\left\|m_{\hbar}(\mu)\right\|$ for all $\hbar \in \mathbb{R}$. By the above arguments, however, $m_{\hbar}$ acts faithfully on the two sub- $C^{*}$-algebras $C^{*}\left(\mathcal{M}\left(E_{\alpha}\right)_{a}, \star_{\hbar}\right)$ and $C^{*}\left(\mathcal{M}\left(E_{\alpha}\right)_{d}, \star_{\hbar}\right)$ of $C^{*}\left(\mathcal{M}\left(E_{\alpha}\right), \star_{\hbar}\right)$, and hence the above proper continuity is valid for each $\mu \in \mathcal{M}\left(E_{\alpha}\right)_{a} \cup \mathcal{M}\left(E_{\alpha}\right)_{d}$.

Taking the inductive limit of the $C^{*}$-algebras $C^{*}\left(\mathcal{M}\left(E_{\alpha}\right), \star_{\hbar}\right), \alpha \in I$, we arrive at the enveloping $C^{*}$-algebra $C^{*}\left(\mathcal{M}(E), \star_{\hbar}\right)$ of the whole Banach-*-algebra $\left(\mathcal{M}(E), \star_{\hbar}\right)$, the $C^{*}$-norm of which is denoted by $\|\cdot\|_{\hbar}$, too. Also here we have that $C^{*}\left(\mathcal{M}(E)_{d}, \star_{\hbar}\right)=\mathcal{W}(E, \hbar \sigma)$ is a sub$C^{*}$-algebra, and $C^{*}\left(\mathcal{M}(E)_{c}, \star_{\hbar}\right)$ is a closed $*$-ideal of $C^{*}\left(\mathcal{M}(E), \star_{\hbar}\right)$. But analogously as for the Banach-*-algebras $\left(\mathcal{M}\left(E_{\alpha}\right)_{a}, \star_{\hbar}\right), \alpha \in I$, there is no inductive limit of the absolutely continuous measure $C^{*}$-algebras $C^{*}\left(\mathcal{M}\left(E_{\alpha}\right)_{a}, \star_{\hbar}\right), \alpha \in I$. A certain generalization of the above 1:1:1:1-correspondence to infinite dimensional $(E, \sigma)$ is found in [46].

We now turn to representations of our measure Banach-*-algebra $\left(\mathcal{M}(E), \star_{\hbar}\right)$. By the Subsections 2.2 and 5.2, especially by equation (5.3), each representation (class)

$$
\Pi_{\hbar} \in \operatorname{rep}\left(\mathcal{M}(E)_{d}, \star_{\hbar}\right)=\operatorname{rep}(\mathcal{W}(E, \hbar \sigma)) \quad \text { with } \quad \Pi_{\hbar} \leq \Pi_{\hbar}^{\mathrm{reg}}
$$

gives rise to the projective unitary group representation (class)

$$
E \ni f \longmapsto \pi_{\hbar}(f)=\Pi_{\hbar}\left(W^{\hbar}(f)\right)=\Pi_{\hbar}(\delta(f)),
$$

which is continuous on each finite dimensional subspace $E_{\alpha}$ of $E$ with respect to the $\sigma$-strong (equivalently $\sigma$-weak) topology on the $\mathrm{W} *$-algebra ${\overline{\Pi_{\hbar}(\mathcal{W}(E, \hbar \sigma))}}^{w}$. Since $\sigma$ is jointly continuous on each $E_{\alpha}$, it is obvious that $\Pi_{\hbar}$ may be extended by integration in a weak operator topology, like

$$
\mu \longmapsto \Pi_{\hbar}(\mu):=\int_{E} d \mu(f) \pi_{\hbar}(f) \in{\overline{\Pi_{\hbar}(\mathcal{W}(E, \hbar \sigma))}}^{w},
$$

to a representation of each Banach-*-algebra $\left(\mathcal{M}\left(E_{\alpha}\right), \star_{\hbar}\right), \alpha \in I$. Finally, by taking the inductive limit over $\alpha \in I$ and by using a $\|\cdot\|_{1}$-density argument, one arrives at a representation of the whole Banach-*-algebra $\left(\mathcal{M}(E), \star_{\hbar}\right)$, denoted by the same symbol. If $\Pi_{\hbar} \leq \Pi_{\hbar}^{\tau}$ (requiring $\Pi_{\hbar}$ to be $\tau$-continuous), for some locally convex $\tau \in \mathcal{T}(E, \sigma)$, then the extension in equation (5.8) may be performed for all Borel measures $\mu \in M^{\tau}(E)$, in which case $\Pi_{\hbar}$ is only a linear mapping; only its restriction to $\mathcal{M}(E)$ is a $*$-homomorphism. Note that for an only regular $\Pi_{\hbar}$ the linear extension to $M^{\tau}(E)$ does not work.

Since every representation $\Pi_{\hbar}$ from the sub- $C^{*}$-algebra $C^{*}\left(\mathcal{M}(E)_{d}, \star_{\hbar}\right)=\mathcal{W}(E, \hbar \sigma)$ may be extended to a representation on $C^{*}\left(\mathcal{M}(E), \star_{\hbar}\right)$ with in general a larger representation Hilbert space, we may regard subsequently only representations (resp. equivalence classes of representations) of $\left(\mathcal{M}(E), \star_{\hbar}\right)$. So, when writing $\Pi_{\hbar}$, we henceforth mean an element of $\operatorname{rep}\left(\mathcal{M}(E), \star_{\hbar}\right)=$ $\operatorname{rep}\left(C^{*}\left(\mathcal{M}(E), \star_{\hbar}\right)\right)$.

To avoid the abuse of "regular" and to indicate the construction method, we make the following convention for infinite dimensional $E$.

Definition 2 (Integration type representations of $\left(\mathcal{M}(E), \star_{\hbar}\right)$ ). Under an integration type representation $\Pi_{\hbar}$ of $\left(\mathcal{M}(E), \star_{\hbar}\right)$ (or of $\left(C^{*}\left(\mathcal{M}(E), \star_{\hbar}\right)\right.$ ) we understand a representation, 
which extends either a regular or a $\tau$-continuous representation $\left(\Pi_{\hbar}, \mathcal{H}_{\hbar}\right)$ of the $C^{*}$-Weyl algebra $\mathcal{W}(E, \hbar \sigma)=C^{*}\left(\mathcal{M}(E)_{d}, \star_{\hbar}\right)$, using equation (5.8). By construction, the representation Hilbert space is still $\mathcal{H}_{\hbar}$.

(Recall that for infinite dimensional $E$ "regularity" of a representation of the Weyl algebra $\mathcal{W}(E, \hbar \sigma)=C^{*}\left(\mathcal{M}(E)_{d}, \star_{\hbar}\right)$ is weaker than " $\tau$-continuity" (in contradistinction to the finite dimensional case), but both properties allow to extend the weak integration over test functions from discrete to more general measures.)

The set of (quasi-equivalence classes of) integration type representations is denoted nevertheless by repreg $\left(\mathcal{M}(E), \star_{\hbar}\right)=\operatorname{rep}_{\text {reg }}\left(C^{*}\left(\mathcal{M}(E), \star_{\hbar}\right)\right)$ (to have a short subscript). The integrated extensions of the largest regular resp. $\tau$-continuous representation, namely of $\Pi_{\hbar}^{\text {reg }}$ resp. of $\Pi_{\hbar}^{\tau}$, are denoted by the old symbols.

Especially, when writing $\Pi_{\hbar} \leq \Pi_{\hbar}^{\mathrm{reg}}$ or $\Pi_{\hbar} \leq \Pi_{\hbar}^{\tau}$, we indicate that $\Pi_{\hbar}$ is of integration type, too.

Lemma 6. For $\hbar \neq 0$ the following assertions are valid:

(a) Let $\alpha \in I_{\sigma}$ (i.e. $\sigma$ is non-degenerate on $E_{\alpha}$, Section 3). Then $C^{*}\left(\mathcal{M}\left(E_{\alpha}\right)_{a}, \star_{\hbar}\right)$ is simple (a statement implying the well-known simplicity of the Weyl algebra $\mathcal{W}\left(E_{\alpha}, \hbar \sigma\right)$ by means of Lemma 5).

(b) Let $\sigma$ be non-degenerate on $E$. Then we have $\left\|\Pi_{\hbar}(\mu)\right\|=\left\|\Pi_{\hbar}^{\mathrm{reg}}(\mu)\right\|, \mu \in \mathcal{M}(E)$, for every subrepresentation $\Pi_{\hbar} \leq \Pi_{\hbar}^{\mathrm{reg}}$. This is also valid when we replace $E$ by $E_{\alpha}$ with $\alpha \in I_{\sigma}$.

Proof. [13] ensures that the regular part $\Pi_{\hbar}$ of any representation $\Psi_{\hbar}=\Pi_{\hbar} \oplus \Pi_{\hbar}^{N}$ of $\left(\mathcal{M}\left(E_{\alpha}\right), \star_{\hbar}\right)$ is a direct sum of identical copies of the irreducible Schrödinger representation, a generalized von Neumann uniqueness result. Thus $\left\|\Pi_{\hbar}(\mu)\right\|$ has the same value for every integration type representation $\Pi_{\hbar}$. Moreover, $\left\|\Pi_{\hbar}(\mu)\right\|=\|\mu\|_{\hbar}$ for all $\mu \in \mathcal{M}\left(E_{\alpha}\right)_{a}$, since $\Pi_{\hbar}^{N}\left(\mathcal{M}\left(E_{\alpha}\right)_{a}\right)=0$. For part (b) take the inductive limit over $\alpha \in I_{\sigma}$.

Let us finally give the classical case $(\hbar=0)$ a special treatment. Recall that $\|\widehat{\mu}\|_{0}$ denotes the supremum norm of the continuous phase space function $\widehat{\mu}$. $\|\widehat{\mu}\|_{0} \leq\|\mu\|_{1}$ for all $\mu \in M^{\tau}(E)$, which may be shown directly, and which is for $\mu \in \mathcal{M}(E)$ a consequence of the continuity of *-homomorphisms [61, Proposition I.5.2].

Proposition 3. The function algebra, given by the Fourier transformed $\left(\mathcal{M}(E), \star_{0}\right)$, is *isomorphic to the largest $\tau$-continuous representation class $\Pi_{0}^{\tau} \in \operatorname{rep}_{\text {reg }}\left(C^{*}\left(\mathcal{M}(E), \star_{0}\right)\right)$, restricted to $\left(\mathcal{M}(E), \star_{0}\right)$, for an arbitrary locally convex topology $\tau$ on $E$, what essentially amounts to the equality of the $C^{*}$-norms $\left\|\Pi_{0}^{\tau}(\mu)\right\|=\|\widehat{\mu}\|_{0}$ for all $\mu \in \mathcal{M}(E)$. (For represented algebra elements we use the operator norm.)

Furthermore, $\Pi_{0}^{\tau}$ acts faithfully on the sub-C $C^{*}$-algebras $C^{*}\left(\mathcal{M}\left(E_{\alpha}\right)_{a}, \star_{0}\right)$, for every $\alpha \in I$, and on $C^{*}\left(\mathcal{M}(E)_{d}, \star_{0}\right)$. In terms of the $C^{*}$-norm $\|\cdot\|_{0}$ of $C^{*}\left(\mathcal{M}(E)\right.$, $\left.\star_{0}\right)$, that means

$$
\|\mu\|_{0}=\left\|\Pi_{0}^{\tau}(\mu)\right\|=\|\widehat{\mu}\|_{0}, \quad \forall \mu \in \mathcal{M}(E)_{d} \bigcup\left(\bigcup_{\alpha} \mathcal{M}\left(E_{\alpha}\right)_{a}\right) .
$$

Proof. Let $\mu \in \mathcal{M}\left(E_{\alpha}\right)$. As mentioned in Subsection 5.2 the characteristic funtions $C_{\omega}$ of the states $\omega$ from the folium $\mathcal{F}_{0}^{\tau}$ associated with $\Pi_{0}^{\tau}$ are $\tau$-continuous, positive-definite functions on $E$. By Bochner's theorem (which is not valid for infinite dimensional $E$ because of the lack of local compactness) for the restriction of $C_{\omega}$ to $E_{\alpha}$ there exists a unique (positive) probability measure $\rho_{\omega}$ on $E_{\alpha}^{\prime}$ such that $C_{\omega}(f)=\int_{E_{\alpha}^{\prime}} d \rho_{\omega}[F] \exp \{i F(f)\}$ for all $f \in E_{\alpha}$. We conclude that

$$
\left\langle\omega ; \mu^{*} \star_{0} \mu\right\rangle=\int_{E_{\alpha}} d \mu^{*}(f) \int_{E_{\alpha}} d \mu(g) C_{\omega}(f+g)=\int_{E_{\alpha}^{\prime}} d \rho_{\omega}[F]|\widehat{\mu}[F]|^{2} \leq\|\widehat{\mu}\|_{0}^{2} .
$$


Since for every $F \in E_{\tau}^{\prime}$ there is a state in $\mathcal{F}_{0}^{\tau}$ with characteristic function $E \ni f \mapsto \exp \{i F(f)\}$ (cf. again Subsection 5.2), we conclude that $\left\|\Pi_{0}^{\tau}(\mu)\right\|^{2}=\sup \left\{\left\langle\omega ; \mu^{*} \star_{0} \mu\right\rangle \mid \omega \in \mathcal{F}_{0}^{\tau}\right\}=\|\widehat{\mu}\|_{0}^{2}$ (recall that $\mathcal{F}_{0}^{\tau}$ consists of the normal states on ${\overline{\Pi_{\hbar}(\mathcal{W}(E, 0))}}^{w})$. Taking the inductive limit over $\alpha \in I$ we get $\left\|\Pi_{0}^{\tau}(\mu)\right\|=\|\widehat{\mu}\|_{0}$ for all $\mu \in \mathcal{M}(E)$. The rest is immediate with Lemma 8 of the Appendix.

Lemma 7. For $\mu \in M^{\tau}(E)^{+}$we have $\|\mu\|_{1}=\mu(E)=\widehat{\mu}[0]=\|\widehat{\mu}\|_{0}$.

Proof. For $\mu \in M^{\tau}(E)^{+}$it follows that $\widehat{\mu}$ is a continuous positive-definite function on the additive group $E_{\tau}^{\prime}$. Hence $|\widehat{\mu}[F]| \leq \widehat{\mu}[0]$ for all $F \in E_{\tau}^{\prime}$, e.g. [32, (32.4)], and thus $\|\widehat{\mu}\|_{0}=\widehat{\mu}[0]$. On the other side, $\mu$ being positive ensures $\mu=|\mu|$, and hence $\|\mu\|_{1}=\mu(E)=\widehat{\mu}[0]$.

\section{Extended Weyl quantization as strict deformation quantization (of $C^{*}$-type)}

\subsection{Families of representations, quantization maps}

Up to now we have developed the Banach-*-algebra version $\left(Q_{\hbar}^{B}\right)_{\hbar \in \mathbb{R}}$ of strict deformation quantization (in Theorem 4) for the generalized Weyl quantization. The transition to the original $C^{*}$-type version from Definition 1 is carried through in a second step by the selection of a family of (quasi-equivalence classes of) representations

$$
\Pi \equiv\left(\Pi_{\hbar}\right)_{\hbar}:=\left\{\Pi_{\hbar} \in \operatorname{rep}\left(\mathcal{M}(E), \star_{\hbar}\right) \mid \hbar \neq 0\right\} .
$$

This being given, we define for every $\hbar \neq 0$ the family of quantization maps by

$$
\widehat{\mu} \longmapsto Q_{\hbar}^{\Pi}(\widehat{\mu}):=\Pi_{\hbar}(\underbrace{Q_{\hbar}^{B}(\mu)}_{=\mu})=\Pi_{\hbar}(\mu) .
$$

As is common for Weyl quantization, we start from phase space functions $\widehat{\mu}: \mathrm{P} \rightarrow \mathbb{C}$ (with $\left.\mathrm{P} \equiv E_{\tau}^{\prime}\right)$ instead of the associated measures $\mu \in \mathcal{M}(E)$ resp. $M^{\tau}(E)$.

Since Fourier transformation is a bijection, the quantization map $Q_{\hbar}^{\Pi}$ is well-defined on $\widehat{\mathcal{M}}\left(E_{\tau}^{\prime}\right)$, even on $\widehat{M}\left(E_{\tau}^{\prime}\right)$, provided $\Pi_{\hbar} \leq \Pi_{\hbar}^{\tau}$. Recall from Definition 2 , that $\Pi_{\hbar}$ may be an integration type representation

$$
\Pi_{\hbar}(\mu)=\int_{E} d \mu(f) \underbrace{\Pi_{\hbar}\left(W^{\hbar}(f)\right)}_{=\pi_{\hbar}(f)} \in{\overline{\Pi_{\hbar}(\mathcal{W}(E, \hbar \sigma))}}^{w} .
$$

For a true strict deformation quantization one has to restrict the domain of definition of the quantization maps $Q_{\hbar}^{\Pi}, \hbar \neq 0$, to a Poisson algebra $\widehat{\mathcal{P}}=\mathbb{F} \mathcal{P}$ of phase space functions. We may choose $\mathcal{P}$ as any measure Poisson algebra from Theorem 3, whose Fourier transformations are treated in Theorem 5 .

For each $\hbar$ the images $Q_{\hbar}^{\Pi}(\widehat{\mu})=\Pi_{\hbar}(\mu)$ have to be operators in a $C^{*}$-algebra, for which we take e.g. the smallest $C^{*}$-algebra $\mathcal{A}^{\hbar}$ comprising the represented Banach-*-algebra $\Pi_{\hbar}\left(\mathcal{M}(E)\right.$, $\left.\star_{\hbar}\right)$. We elaborate this for selected families $\Pi \equiv\left(\Pi_{\hbar}\right)_{\hbar}$ of representations.

As mentioned previously, we have the norm estimations

$$
\left\|\Pi_{\hbar}(\mu)\right\| \leq\|\mu\|_{1}, \quad \forall \hbar \in \mathbb{R}, \quad \mu \in \mathcal{M}(E)
$$

where $\|\cdot\|$ are the $C^{*}$-norms of the different $\mathcal{A}^{\hbar}$. From these estimations the Dirac and the von Neumann conditions follow easily, see Subsection 6.2. But, whereas Rieffel's condition has been trivially fulfilled in the Banach-*-deformation quantization $\left(Q_{\hbar}^{B}\right)_{\hbar \in \mathbb{R}}$ of Theorem 4 , relation (6.3) 
does not provide a simple deduction of the continuity of $\mathbb{R} \ni \hbar \mapsto\left\|Q_{\hbar}^{\Pi}(\widehat{\mu})\right\|=\left\|\Pi_{\hbar}(\mu)\right\|$ with the appropriate $C^{*}$-norms for the different values of $\hbar$ (Rieffel's condition).

For the representation family $\Pi=\left(\Pi_{\hbar}\right)_{\hbar}$ we consider $\Pi^{\#}:=\left(\Pi_{\hbar}^{\#}\right)_{\hbar}$ with $\# \in\{$ reg, il, $\tau\}$, $\tau \in \mathcal{T}(E, \sigma)$. We write $\Pi \leq \Pi^{\#}$, if $\Pi_{\hbar} \leq \Pi_{\hbar}^{\#}$ for every $\hbar \neq 0$. A family $\Pi \leq \Pi^{\tau}$ may be linearly extended from the universal $\left(\mathcal{M}(E), \star_{\hbar}\right)$ to all of $M^{\tau}(E)$, by using the integration type method equation (5.8). Thus, $\left\|\Pi_{\hbar}(\mu)\right\| \leq\|\mu\|_{1}$ in any case. If in $\Pi=\left(\Pi_{\hbar}\right)_{\hbar}$ each $\Pi_{\hbar}$ acts faithfully on a sub- $C^{*}$-algebra $\mathcal{B}^{\hbar}$ of the enveloping $C^{*}$-algebra $C^{*}\left(\mathcal{M}(E), \star_{\hbar}\right)$, then we call $\Pi$ "faithful on $\left(\mathcal{B}^{\hbar}\right)_{\hbar \neq 0} "$.

Up to now, a family $\Pi=\left(\Pi_{\hbar}\right)_{\hbar}$ of quasi-equivalence classes of representations was defined for the values $\hbar \neq 0$, only. We add for the classical case $\hbar=0$ the Fourier transformation $\mathbb{F}$ as the representation $\Pi_{0}$, which by Proposition 3 is $*$-isomorphic to the integration type representation $\Pi_{0}^{\tau} \in \operatorname{rep}_{\text {reg }}\left(\mathcal{M}(E), \star_{0}\right)$, for each $\tau$. Let us write then

$$
Q_{0}^{\Pi}(\widehat{\mu}):=\Pi_{0}(\mu)=\widehat{\mu}, \quad \forall \mu \in \mathcal{M}(E) \quad \text { resp. } M^{\tau}(E) .
$$

We subsequently make the identification $\left\|Q_{0}^{\Pi}(\widehat{\mu})\right\|=\|\widehat{\mu}\|_{0}$, with the sup-norm $\|\cdot\|_{0}$, dropping occasionally the subscript "0".

\subsection{Dirac's and von Neumann's conditions}

As an immediate consequence of inequality (6.3) we obtain from Theorem 4 the main part of a strict quantization.

Theorem 6. For each family $\Pi=\left(\Pi_{\hbar}\right)_{\hbar}$ of representations, not necessarily of integration type:

(a) [Dirac] $\lim _{\hbar \rightarrow 0}\left\|\left\{Q_{\hbar}^{\Pi}(\widehat{\mu}), Q_{\hbar}^{\Pi}(\widehat{\nu})\right\}_{\hbar}-Q_{\hbar}^{\Pi}(\{\widehat{\mu}, \widehat{\nu}\})\right\|=0$ for all $\mu, \nu \in \mathcal{M}_{\varsigma}^{1}(E)$, with the Poisson bracket $\{\cdot, \cdot\}$ from equation (4.13), and with the $\hbar$-scaled commutators $\{\cdot, \cdot\}_{\hbar}$ from $(2.2)$.

(b) [von Neumann] $\lim _{\hbar \rightarrow 0}\left\|Q_{\hbar}^{\Pi}(\widehat{\mu}) Q_{\hbar}^{\Pi}(\widehat{\nu})-Q_{\hbar}^{\Pi}(\widehat{\mu} \cdot 0 \widehat{\nu})\right\|=0$ for all $\mu, \nu \in \mathcal{M}(E)$.

That means that the Dirac and von Neumann conditions are valid for all $\widehat{\mu}$ and $\widehat{\nu}$ contained in any sub-Poisson algebra $\tilde{\mathcal{P}}$ of $\left(\widehat{\mathcal{M}}_{\varsigma}^{\infty}\left(E_{\tau}^{\prime}\right),{ }_{0},\{\cdot, \cdot\}\right)$, where we think especially on the Fourier transformed measure Poisson algebras $\tilde{\mathcal{P}}:=\widehat{\mathcal{P}}=\mathbb{F} \mathcal{P}$, with $\mathcal{P}$ from Theorem 3 (including the case $\left.\mathcal{P}=\mathcal{M}_{\varsigma}^{\infty}(E)\right)$.

\subsection{On Rieffel's continuity condition}

In Rieffel's condition the norms of the representations $\Pi_{\hbar}$ of $\left(\mathcal{M}(E)\right.$, $\left.\star_{\hbar}\right)$ are compared with each other for different $\hbar \in \mathbb{R}$, which causes some complications. This problem does not arise for the usual $C^{*}$-Weyl algebras, corresponding to the discrete measures. We include this previously discussed case into the present method of dealing with representations.

Example 1 (Discrete case). For each family $\Pi=\left(\Pi_{\hbar}\right)_{\hbar}$, which is faithful on $\left(C^{*}\left(\mathcal{M}(E)_{d}, \star_{\hbar}\right)=\right.$ $\mathcal{W}(E, \hbar \sigma))_{\hbar \neq 0}$, the present quantization maps $\left(Q_{\hbar}^{\Pi}\right)_{\hbar \in \mathbb{R}}$ are $*$-isomorphic to the previous $C^{*}$ algebraic quantization maps of the strict deformation quantization in Theorem 1 (use Proposition 3 for $\hbar=0)$. Candidates $\mathcal{P}$ for a measure Poisson algebra are given by $\mathcal{M}(E)_{d f} \cong \Delta(E, 0)$, and by $\mathcal{M}(E)_{d} \cap \mathcal{M}_{\varsigma}^{\infty}(E)$ from Theorem 3 (already used in [14]).

The present, representation dependent quantization procedure, aims however at a larger class of $C^{*}$-algebraic quantizations, founded on the enlarged measure space $\mathcal{M}(E) \supset \mathcal{M}(E)_{d}$. Before stating (and proving) the results in detail, let us give a brieve overview. 
Summary 2. Let $\Pi$ be a family of integration type representations of $\left(\mathcal{M}(E), \star_{\hbar}\right)$. Under certain circumstances ( $\sigma$ non-degenerate, or $\Pi$ suitably well-matched), we achieve by our own efforts lower semicontinuity of $\mathbb{R} \ni \hbar \mapsto\left\|Q_{\hbar}^{\Pi}(\widehat{\mu})\right\|=\left\|\Pi_{\hbar}(\mu)\right\|$, for all $\mu \in \mathcal{M}(E)$. Using some results of Rieffel one even gets true continuity for non-degenerate $\sigma$.

Thus, if Rieffel's condition is weakened to lower semicontinuity, then $\left(Q_{\hbar}^{\Pi}\right)_{\hbar \in \mathbb{R}}$ constitutes a strict deformation quantization of each classical Poisson algebra $\widehat{\mathcal{P}}=\mathbb{F} \mathcal{P}$ of phase space functions, which is mentioned in Theorem 3.

In virtue of Lemma 6(b) the case of a non-degenerate $\sigma$ is simpler than that of a degenerate one, since only for degenerate $\sigma$ one may have that $\left\|\Pi_{\hbar}(\mu)\right\|<\left\|\Pi_{\hbar}^{\mathrm{reg}}(\mu)\right\|$, for some $\mu \in \mathcal{M}(E)$, in a proper subrepresentation $\Pi_{\hbar}<\Pi_{\hbar}^{\text {reg }}$. The following two Subsections treat technical questions of this nature.

\subsubsection{Non-degenerate symplectic form $\sigma$}

Let us assume $\sigma$ non-degenerate on the infinite dimensional test function space $E$. The proof of the next result is deferred to Appendix B.

Proposition 4 (Lower semicontinuity). Let $\Pi \leq \Pi^{\text {reg }}$ be an arbitrary family of integration type representations of $\left(\mathcal{M}(E), \star_{\hbar}\right)$ (formed with a non-degenerate $\sigma$ ). Then $\mathbb{R} \ni \hbar \mapsto\left\|Q_{\hbar}^{\Pi}(\widehat{\mu})\right\|=$ $\left\|\Pi_{\hbar}(\mu)\right\|$ is lower semicontinuous for every $\mu \in \mathcal{M}(E)$.

We want to relate our investigation to Rieffel's works $[26,10]$. This may be carried out for non-degenerate $\sigma$, since only in this case the matrix $J$ in equation (6.4) below may be related to $\sigma$ via a transformation of the variables.

Let $\alpha \in I_{\sigma}$ (i.e. $\sigma$ acts non-degenerately on the finite dimensional subspaces $E_{\alpha}$, cf. beginning of Section 3). In equation (4.4) we see how the Fourier transformation of the twisted convolution products leads to the deformed or Moyal products for functions [12]. The latter products $\hbar$ may be formulated for measures $\mu$ and $\nu$ on $E_{\alpha}$ in terms of oscillatory integrals as follows

$$
\widehat{\mu} \cdot \hbar \widehat{\nu}[F]=\int_{E_{\alpha}^{\prime}} d G \int_{E_{\alpha}^{\prime}} d H \widehat{\mu}[F+\hbar J G] \widehat{\nu}[F+H] \exp \{i G \cdot H\}
$$

where $J$ is an anti-symmetric matrix arising from $\sigma$ and $G \cdot H$ is an inner product on $E_{\alpha}^{\prime}$. Rieffel shows the continuity of $\mathbb{R} \ni \hbar \mapsto\left\|\widehat{\Pi}_{\hbar}^{L^{2}}(\widehat{\mu})\right\|$ for $\mu \in \mathcal{M}_{\varsigma}^{\infty}\left(E_{\alpha}\right)$ for the Fourier transforms $\widehat{\Pi}_{\hbar}^{L^{2}}$ of the $\mathrm{L}^{2}$-representations $\Pi_{\hbar}^{L^{2}}$ from Subsection 5.4, a specific, well-matched $\mathrm{L}^{2}$-representation family. For $\hbar=0$ observe that $\widehat{\mu}$ acts as multiplicator on the Fourier transformed $\mathrm{L}^{2}$-space, the operator norm of which is just given by its sup-norm $\|\widehat{\mu}\|_{0}=\left\|\widehat{\Pi}_{0}^{L^{2}}(\widehat{\mu})\right\|=\left\|\Pi_{0}^{L^{2}}(\mu)\right\|$. Thus in virtue of Lemma 6(b) and by taking the inductive limit over $\alpha \in I_{\sigma}$ one arrives at the continuity of $\mathbb{R} \ni \hbar \mapsto\left\|\Pi_{\hbar}(\mu)\right\|$ for all $\mu \in \mathcal{M}(E)$, for arbitrary families $\Pi \leq \Pi^{\text {reg }}$ of integration type representation classes.

\subsubsection{Degenerate $\sigma$, well-matched representation families}

Let us now allow for a degenerate, non-trivial $\sigma$ on $E$. Because of Lemma 6(b) a norm deficit $\left\|\Pi_{\hbar}(\mu)\right\|<\left\|\Pi_{\hbar}^{\mathrm{reg}}(\mu)\right\|, \mu \in \mathcal{M}(E)$, is possible in a proper subrepresentation $\Pi_{\hbar}<\Pi_{\hbar}^{\mathrm{reg}}$. Thus we need a certain compatibility condition between representations $\Pi_{\hbar}$, with different $\hbar \neq 0$, within a family $\Pi=\left(\Pi_{\hbar}\right)_{\hbar}$. Otherwise, one could not expect continuity properties for $\mathbb{R} \ni \hbar \mapsto\left\|\Pi_{\hbar}(\mu)\right\|$.

Definition 3 (Well-matched families of representation classes). Let $\tau \in \mathcal{T}(E, \sigma)$ be a locally convex topology. A family $\Pi=\left(\Pi_{\hbar}\right)_{\hbar}$ is called well-matched (with respect to $\tau$ ), if the following assertions are valid: 
(A) For each $\hbar \neq 0$ there is an $\mathbb{R}$-linear $\tau$-homeomorphism $T_{\hbar}$ on $E$ satisfying $\sigma\left(T_{\hbar} f, T_{\hbar} g\right)=$ $\hbar \sigma(f, g)$ for all $f, g \in E$. (By Lemma 1 then there exists a unique $*$-isomorphism $\beta_{\hbar}$ from $\mathcal{W}(E, \hbar \sigma)$ onto $\mathcal{W}(E, \sigma)$ with $\beta_{\hbar}\left(W^{\hbar}(f)\right)=W^{1}\left(T_{\hbar} f\right)$, for all $f \in E$.)

(B) For $\hbar=1$ we have $\Pi_{1} \leq \Pi_{1}^{\tau}$ and $\Pi_{\hbar}=\Pi_{1} \circ \beta_{\hbar}$ for all $\hbar \neq 0$, with the representations acting on $\mathcal{W}(E, \hbar \sigma)$ (relations which are extensible via the integration type construction to $C^{*}\left(\mathcal{M}(E), \star_{\hbar}\right)$ according to Definition 2$)$.

(C) Moreover, $\hbar \mapsto T_{\hbar} f$ is assumed $\tau$-continuous with $\lim _{\hbar \rightarrow 0} T_{\hbar} f=0$, for all $f \in E$.

Instead of $\tau$-continuous representations one may also treat merely regular ones. Then the $\mathbb{R}$-linear bijections $T_{\hbar}$ on $E$ are demanded to leave each finite dimensional subspace invariant, so that part $(\mathrm{C})$ is reasonable, and one assumes $\Pi_{1} \leq \Pi_{1}^{\mathrm{reg}}$.

The dual mapping $\beta_{\hbar}^{*}$ is an affine bijection from the folium $\mathcal{F}_{\hbar=1}^{\tau}$ onto the folium $\mathcal{F}_{\hbar}^{\tau}$, and consequently $\beta_{\hbar}$ extends $\sigma$-strong continuously to a $*$-isomorphism from the $\mathrm{W} *$-algebra ${\overline{\Pi_{\hbar}^{\tau}(\mathcal{W}(E, \hbar \sigma))}}^{w}$ onto the $\mathrm{W}$ *-algebra ${\overline{\Pi_{\hbar=1}^{\tau}(\mathcal{W}(E, \sigma))}}^{w}$, which in addition maps $\Pi_{\hbar}^{\tau}\left(\mathcal{M}(E), \star_{\hbar}\right)$ onto $\Pi_{\hbar=1}^{\tau}\left(\mathcal{M}(E), \star_{1}\right)$ and $\Pi_{\hbar}^{\tau}\left(C^{*}\left(\mathcal{M}(E), \star_{\hbar}\right)\right)$ onto $\Pi_{\hbar=1}^{\tau}\left(C^{*}\left(\mathcal{M}(E), \star_{1}\right)\right)$. Some examples of $T_{\hbar}$ fulfilling the above Definition are given in Subsection 2.3. (If in concrete situations such $T_{\hbar}$ are only given for $\hbar$ in a subset $J \subset \mathbb{R}$ - as in Subsection 2.1-, then the subsequent results remain valid by restricting $\hbar \in \mathbb{R}$ to $\hbar \in J$.)

The notion of $E_{\rho}^{\prime}$-invariance, used in our next result, is introduced in Appendix A, the proof is found in Appendix B.

Proposition 5 (Lower semicontinuity). Let $\Pi \leq \Pi^{\text {reg }}$ be a family of integration type representations.

(a) Assume $\Pi$ well-matched. Then $\hbar \mapsto\left\|Q_{\hbar}^{\Pi}(\widehat{\mu})\right\|$ is lower semicontinuous on $\mathbb{R} \backslash\{0\}$, for every $\mu \in \mathcal{M}(E)$, and we have $\lim _{\hbar \rightarrow 0}\left\|Q_{\hbar}^{\Pi}(\widehat{\mu})\right\|=\|\widehat{\mu}\|_{0}$ for all $\mu \in \mathcal{M}(E)^{+}$.

Suppose in addition a locally convex topology $\rho$ on $E$ so that either each $\Pi_{\hbar}$ is $E_{\rho}^{\prime}$-invariant, or that $\Pi_{\hbar=1}$ is partially $E_{\rho}^{\prime}$-invariant and $E_{\rho}^{\prime} \circ T_{\hbar}^{-1}=E_{\rho}^{\prime}$, for each $\hbar \neq 0$. Then the above lower semicontinuity is valid on all of $\mathbb{R}$ (now including the origin).

(b) Let $\Pi$ be well-matched with reference to $\tau$ (thus especially $\Pi \leq \Pi^{\tau}$ ), and let $\rho \leq \tau$ with $\tau \in \mathcal{T}(E, \sigma)$ a locally convex topology. Then the assertions of part (a) are even valid for $\mu \in M^{\tau}(E)$.

Examples are provided, if $\Pi^{\mathrm{reg}}$ is well-matched and is $E_{\rho}^{\prime}$-invariant, for every locally convex $\rho$ on $E$, and also $\Pi^{\tau}$ is well-matched and $E_{\tau}^{\prime}$-invariant.

Let us now investigate some circumstances under which the lower semicontinuity may be strengthend to proper continuity of $\mathbb{R} \ni \hbar \mapsto\left\|Q_{\hbar}^{\Pi}(\widehat{\mu})\right\|$. We suppose a $\tau$-well-matched family $\Pi$ with $E_{\rho}^{\prime}$-invariance, as in the above Proposition. It is immediately checked that $\mu \mapsto$ $\limsup \left\|\Pi_{\hbar}(\mu)\right\|$ defines a semi- $C^{*}$-norm on the Banach-*-algebra $\left(\mathcal{M}(E), \star_{\hbar_{0}}\right)$. Consequently, $\hbar \rightarrow \hbar_{0}$

$\limsup _{\hbar \rightarrow \hbar_{0}}\left\|\Pi_{\hbar}(\mu)\right\| \leq\|\mu\|_{\hbar_{0}}$ for each $\hbar_{0} \in \mathbb{R}$, since $\|\mu\|_{\hbar_{0}}$ is the $C^{*}$-norm of the enveloping $C^{*}$ algebra $C^{*}\left(\mathcal{M}(E), \star_{\hbar_{0}}\right)$ as in Subsection 5.4. Now equation (B.2) yields:

Example 2. Let $\mu \in \mathcal{M}(E)$. If one is able to show that $\left\|\Pi_{\hbar}(\mu)\right\|=\|\mu\|_{\hbar}$ for all $\hbar \in \mathbb{R}$, then $\mathbb{R} \ni \hbar \mapsto\left\|Q_{\hbar}^{\Pi}(\widehat{\mu})\right\|$ is continuous, that is, Rieffel's condition is satisfied for this $\mu$.

This observation leads to the question, if there exist well-matched families $\Pi=\left(\Pi_{\hbar}\right)_{\hbar}$, which are faithful on the enveloping $C^{*}$-algebras $C^{*}\left(\mathcal{M}(E), \star_{\hbar}\right), \hbar \in \mathbb{R}$ (here including $\hbar=0$ ), resp. on 
parts of it? We know already from Proposition 3 that the representation of the classical observables $\Pi_{0}$ is faithful on $\mathcal{W}(E, 0)=C^{*}\left(\mathcal{M}(E)_{d}, \star_{0}\right)$ and on each $C^{*}\left(\mathcal{M}\left(E_{\alpha}\right)_{a}, \star_{0}\right), \alpha \in I$. In [14] are used similar arguments for proving the referred Theorem 1.

Let $\alpha \in I$ be fixed, and suppose $\Pi_{\hbar=1}$ faithful on $C^{*}\left(\mathcal{M}\left(E_{\alpha}\right)_{a}, \star_{1}\right)$. Here we suppose the family $\Pi=\left(\Pi_{\hbar}\right)_{\hbar}$ to be well-matched with respect to the regularity condition or with respect to $\tau$, but with $T_{\hbar}\left(E_{\alpha}\right)=E_{\alpha}$ for all $\hbar \neq 0$. Then $\Pi=\left(\Pi_{\hbar}\right)_{\hbar}$ is faithful on the $C^{*}$-algebras $\left(C^{*}\left(\mathcal{M}\left(E_{\alpha}\right)_{a}, \star_{\hbar}\right)\right)_{\hbar \in \mathbb{R}}$ and also on the $C^{*}$-Weyl algebras $\mathcal{W}\left(E_{\alpha}, \hbar \sigma\right)=C^{*}\left(\mathcal{M}\left(E_{\alpha}\right)_{d}, \star_{\hbar}\right), \hbar \in \mathbb{R}$ (for $\hbar \neq 0$ by Lemma 5). Consequently, for all $\hbar \in \mathbb{R}$ it is $\left\|\Pi_{\hbar}(\mu)\right\|=\|\mu\|_{\hbar}\left(=\|\widehat{\mu}\|_{0}\right.$ in case of $\hbar=0)$, and by Example 2 Rieffel's condition is valid for every measure $\mu \in \mathcal{M}\left(E_{\alpha}\right)_{a} \cup \mathcal{M}\left(E_{\alpha}\right)_{d}$.

If $\Pi_{\hbar=1}$ acts faithfully on each $C^{*}\left(\mathcal{M}\left(E_{\alpha}\right)_{a}, \star_{1}\right), \alpha \in I$, then $\Pi=\left(\Pi_{\hbar}\right)_{\hbar}$ may be $\tau$-wellmatched with possibly $T_{\hbar}\left(E_{\alpha}\right)=E_{\beta}$ for $\alpha \neq \beta$, in order to obtain Rieffel's condition for all $\mu \in \bigcup_{\alpha \in I} \mathcal{M}\left(E_{\alpha}\right)_{a} \cup \mathcal{M}(E)_{d}$

For finite dimensional $E_{\alpha}$ (its dual $E_{\alpha}^{\prime}$ may be treated as part of $E_{\tau}^{\prime}$ ) the Fourier transformed measures $\widehat{\mathcal{M}}\left(E_{\alpha}^{\prime}\right)$ form a proper subspace of the bounded, $\mathbb{C}$-valued, uniformly continuous functions on $E_{\alpha}^{\prime}$. After all, one knows that $\widehat{\mathcal{M}}\left(E_{\alpha}^{\prime}\right)_{a}$ is a $\|\cdot\|_{0}$-dense sub-*-algebra of the $C^{*}$-algebra $\left(\mathrm{C}_{\infty}\left(E_{\alpha}^{\prime}\right),{ }_{0}\right)$ of the continuous functions on $E_{\alpha}^{\prime}$ vanishing at infinity (cf. e.g. [32, (31.5)]).

Especially, for an arbitrary norm $\kappa$ on $E_{\alpha}$ every $\widehat{\mu} \in \widehat{\mathcal{M}}_{\kappa}^{\infty}\left(E_{\alpha}^{\prime}\right)$ is a smooth bounded function on $E_{\alpha}^{\prime}$, all of whose derivatives of all orders are bounded (and so is similar to an element in Rieffel's function class $\mathcal{B}[10])$. However the Poisson algebra $\widehat{\mathcal{M}}_{\varsigma}^{\infty}\left(E_{\alpha}^{\prime}\right)_{a}=\widehat{\mathcal{M}}_{\varsigma}^{\infty}\left(E_{\alpha}^{\prime}\right) \bigcap \widehat{\mathcal{M}}\left(E_{\alpha}^{\prime}\right)_{a}$ covers the infinitely differentiable functions with compact support, resp. the functions of rapid decrease, which are rather different from the almost periodic functions occurring in the usual $C^{*}$-Weyl quantization. Nevertheless we have proved them to be strictly deformation quantizable (including the full Rieffel condition), also when $\sigma$ acts degenerately on $E_{\alpha}$. Note, if $\sigma$ is degenerate on $E_{\alpha}$, the elements of $\widehat{\mathcal{M}}_{\varsigma}^{\infty}\left(E_{\alpha}^{\prime}\right)_{a}$ are not differentiable in all directions, since $\operatorname{ker}(\varsigma) \bigcap E_{\alpha}$ is non-trivial.

But for degenerate $\sigma$ the elements of $\widehat{\mathcal{M}}_{\varsigma}^{\infty}\left(E_{\alpha}^{\prime}\right)_{a}$ are not differentiable in all directions for non-trivial $\operatorname{ker}(\varsigma) \bigcap E_{\alpha}$ (the latter being possible only for degenerate $\sigma$ on $E_{\alpha}$ ).

One apparently has to use mathematical techniques different from ours for efficiently investigating Rieffel's condition, when one wants to quantize all phase space functions, which are the Fourier transforms of $\mu \in \mathcal{M}(E)$, where even the results concerned with measures $\mu$ on finite dimensional test functions spaces $E_{\alpha}$ have to be completed.

\section{A $\quad E_{\rho}^{\prime}$-invariant folia and representations}

Let $\rho$ be a locally convex topology on $E$ with dual space $E_{\rho}^{\prime}$. For each $F \in E_{\rho}^{\prime}$ there exists a unique *-automorphism $\gamma_{\hbar}^{F}$ on the Weyl algebra $\mathcal{W}(E, \hbar \sigma)$, called gauge transformation of the second kind [30,31], such that

$$
\gamma_{\hbar}^{F}\left(W^{\hbar}(f)\right)=\exp \{i F(f)\} W^{\hbar}(f), \quad \forall f \in E .
$$

Definition 4 ( $\boldsymbol{E}_{\boldsymbol{\rho}}^{\prime}$-invariance). A folium $\mathcal{F}_{\hbar} \in \operatorname{fol}(\mathcal{W}(E, \hbar \sigma))$ respectively the associated representation $\Pi_{\hbar} \in \operatorname{rep}(\mathcal{W}(E, \hbar \sigma))$ are called partially $E_{\rho}^{\prime}$-invariant, if there exists at least one state $\omega \in \mathcal{F}_{\hbar}$ such that $\omega \circ \gamma_{\hbar}^{F} \in \mathcal{F}_{\hbar}$ for all $F \in E_{\rho}^{\prime}$.

$\mathcal{F}_{\hbar}$ resp. $\Pi_{\hbar}$ are called (globally) $E_{\rho}^{\prime}$-invariant, if $\mathcal{F}_{\hbar}=\mathcal{F}_{\hbar} \circ \gamma_{\hbar}^{F}$, or equivalently, if $\Pi_{\hbar}=\Pi_{\hbar} \circ \gamma_{\hbar}^{F}$ for all $F \in E_{\rho}^{\prime}$.

Note that for non-degenerate $\sigma$ and $\hbar \neq 0$ every $\mathcal{F}_{\hbar} \in \operatorname{fol}(\mathcal{W}(E, \hbar \sigma))$ is $E_{\rho}^{\prime}$-invariant for the locally convex topology $\rho$ arising from the semi-norms $E \ni f \mapsto|\sigma(g, f)|, g \in E$ (since for $\omega \in \mathcal{F}_{\hbar}$ one has $\left\langle\omega ; W^{\hbar}(-g) . W^{\hbar}(g)\right\rangle \in \mathcal{F}_{\hbar}$ by the definition of a folium), especially $\rho \in \mathcal{T}(E, \sigma)$. 
As mentioned already, the $C^{*}$-Weyl algebra $\mathcal{W}(E, \hbar \sigma)$ is simple, if and only if $\sigma$ is nondegenerate and $\hbar \neq 0$, in which case every representation is faithful. But also for degenerate $\sigma$ we have:

Lemma 8. Let $\rho \leq \tau$ for locally convex $\rho$ and $\tau \in \mathcal{T}(E, \sigma)$. Then $\mathcal{F}_{\hbar}^{\tau}$ is $E_{\rho}^{\prime}$-invariant, and the associated representation $\Pi_{\hbar}^{\tau}$ is faithful on $\mathcal{W}(E, \hbar \sigma)$.

Proof. The $E_{\rho}^{\prime}$-invariance is immediate. For the proof of the case $\hbar=0$ one may assume that $\sigma=0$. So suppose $\hbar \neq 0$. By construction the quotient symplectic form $\sigma_{q}(q(f), q(g)):=\sigma(f, g)$ on the quotient $E / \operatorname{ker}_{\sigma}$ is non-degenerate $\left(\mathrm{ker}_{\sigma}\right.$ is the null space of $\sigma$ from equation (2.4), and $q(f):=f+\operatorname{ker}_{\sigma}$ the quotient map). Hence the $C^{*}$-Weyl algebra $\mathcal{W}\left(E / \operatorname{ker}_{\sigma}, \hbar \sigma_{q}\right)$ is simple, and so every non-degenerate representation of it is faithful.

Let $\Pi^{\prime}$ be a non-degenerate representation of $\mathcal{W}\left(E / \operatorname{ker}_{\sigma}, \hbar \sigma_{q}\right)$. Then $\pi(f):=\Pi^{\prime}\left(W^{\hbar}(q(f))\right)$, $f \in E$, defines a projective unitary representation of the additive group $E$, and hence by Subsection 2.2 there exists a unique representation $\Pi$ of $\mathcal{W}(E, \hbar \sigma)$ with $\Pi\left(W^{\hbar}(f)\right)=\pi(f)$. The separate $\tau$-continuity of $\sigma$ implies that $\operatorname{ker}_{\sigma}$ is closed. Thus on the quotient $E / \operatorname{ker}_{\sigma}$ there exists a canonical locally convex topology $\tau_{q}$ arising from $\tau$, for which the quotient map $q: E \rightarrow E / \operatorname{ker}_{\sigma}$ is continuous and $\sigma_{q}$ is separately continuous (e.g. [39, Proposition V.2.1]). Consequently, if $\Pi^{\prime}$ is $\tau_{q}$-continuous, then $\Pi$ is $\tau$-continuous. Especially, by taking GNS representations every $\omega \in$ $\mathcal{F}_{\hbar}^{\tau_{q}} \in \operatorname{fol}\left(\mathcal{W}\left(E / \operatorname{ker}_{\sigma}, \hbar \sigma_{q}\right)\right)$ extends to a unique state $\varphi \in \mathcal{F}_{\hbar}^{\tau}$ with $\left\langle\varphi ; W^{\hbar}(f)\right\rangle=\left\langle\omega ; W^{\hbar}(q(f))\right\rangle$ for all $f \in E$.

Let us fix a state $\omega \in \mathcal{F}_{\hbar}^{\tau_{q}}$ with associated $\varphi \in \mathcal{F}_{\hbar}^{\tau}$. Then $\varphi_{F}:=\varphi \circ \gamma_{F}^{\hbar} \in \mathcal{F}_{\hbar}^{\tau}$ for all $F \in E_{\rho}^{\prime}$. By construction it follows that $\|A\|_{\hbar} \geq\left\|\Pi_{\hbar}^{\tau}(A)\right\| \geq \sup _{F \in E_{\rho}^{\prime}}\left|\left\langle\varphi_{F} ; A\right\rangle\right|$ for all $A \in \mathcal{W}(E, \hbar \sigma)$. Now we restrict ourselves to arbitrary elements $A=\sum_{k} z_{k} W^{\hbar}\left(f_{k}\right)$ from the commutative sub-*algebra $\Delta\left(\operatorname{ker}_{\sigma}, 0\right)$ (note that $\sigma$ restricted to $\operatorname{ker}_{\sigma}$ vanishes), i.e., with $f_{k} \in \operatorname{ker}_{\sigma}$. Since $q(f)=0$ $\left\langle\varphi ; W^{\hbar}(f)\right\rangle=\left\langle\omega ; W^{\hbar}(0)\right\rangle=1$ for all $f \in \operatorname{ker}_{\sigma}$, yielding, for all $F \in E_{\rho}^{\prime}$,

$$
\begin{aligned}
\left\langle\varphi_{F} ; \sum_{k} z_{k} W^{\hbar}\left(f_{k}\right)\right\rangle & =\sum_{k} z_{k} \exp \left\{i F\left(f_{k}\right)\right\}\left\langle\varphi ; W^{\hbar}\left(f_{k}\right)\right\rangle \\
& =\sum_{k} z_{k} \exp \left\{i F\left(f_{k}\right)\right\}=\left(\sum_{k} z_{k} W_{c}\left(f_{k}\right)\right)[F]
\end{aligned}
$$

with the Weyl functions $W_{c}(f)$ from equation (2.13). By the above estimation we get

$$
\begin{aligned}
\left\|\sum_{k} z_{k} W^{\hbar}\left(f_{k}\right)\right\|_{\hbar} & \geq\left\|\Pi_{\hbar}^{\tau}\left(\sum_{k} z_{k} W^{\hbar}\left(f_{k}\right)\right)\right\| \\
& \geq \sup _{F \in E_{\rho}^{\prime}}\left|\sum_{k} z_{k} \exp \left\{i F\left(f_{k}\right)\right\}\right|=\left\|\sum_{k} z_{k} W_{c}\left(f_{k}\right)\right\|_{0} .
\end{aligned}
$$

But from [31] it is known that $\mathcal{W}\left(\operatorname{ker}_{\sigma}, 0\right)$ is a sub- $C^{*}$-algebra of $\mathcal{W}(E, \hbar \sigma)$, which means that the norm on $\mathcal{W}\left(\operatorname{ker}_{\sigma}, 0\right)$ is just the restriction of the norm on $\mathcal{W}(E, \hbar \sigma)$. With the help of Proposition 1 we conclude that $\left\|\sum_{k} z_{k} W^{\hbar}\left(f_{k}\right)\right\|_{\hbar}=\left\|\sum_{k} z_{k} W_{c}\left(f_{k}\right)\right\|_{0}$. Consequently, $\|A\|=$ $\left\|\Pi_{\hbar}^{\tau}(A)\right\|$ for all $A \in \Delta\left(\operatorname{ker}_{\sigma}, 0\right)$, and thus, $\operatorname{ker}\left(\Pi_{\hbar}^{\tau}\right) \bigcap \mathcal{W}\left(\operatorname{ker}_{\sigma}, 0\right)=\{0\}$. Finally, [30, (4.21.iii)] implies that the closed $*$-ideal $\operatorname{ker}\left(\Pi_{\hbar}^{\tau}\right)$ vanishs, that is, $\Pi_{\hbar}^{\tau}$ is faithful.

Note, the proof ensures that also the direct sum representation $\bigoplus_{F} \Pi_{\varphi} \circ \gamma_{F}^{\hbar} \leq \Pi_{\hbar}^{\tau}$ is faithful, for every $\varphi$ constructed from an $\omega \in \mathcal{F}_{\hbar}^{\tau_{q}}$. Hence there exist many proper subrepresentations of $\Pi_{\hbar}^{\tau}$, which are faithful.

\section{B Proofs of the Propositions 5 and 4}

Let us first treat Proposition 5. We demonstrate part (b), part (a) then is obvious. For $\hbar \neq 0$ let $\mathcal{F}_{\hbar} \in \operatorname{fol}(\mathcal{W}(E, \hbar \sigma))$ be the folium associated with $\Pi_{\hbar} \in \operatorname{rep}(\mathcal{W}(E, \hbar \sigma))$. Then $\beta_{\hbar}^{*}\left(\mathcal{F}_{1}\right)=\mathcal{F}_{\hbar}$, 
and $\beta_{\hbar}$ extends $\sigma$-strongly to the associated $\mathrm{W} *$-algebras. $\omega_{\hbar}:=\omega \circ \beta_{\hbar}$ is $\tau$-continuous for $\omega \in \mathcal{F}_{1} \subseteq \mathcal{F}_{1}^{\tau}$, and thus

$$
\hbar \mapsto\left\langle\omega_{\hbar} ; W^{\hbar}(f)\right\rangle=\left\langle\omega ; \beta_{\hbar}\left(W^{\hbar}(f)\right)\right\rangle=\left\langle\omega ; W^{1}\left(T_{\hbar} f\right)\right\rangle
$$

is continuous on $\mathbb{R} \backslash\{0\}$ with $\lim _{\hbar \rightarrow 0}\left\langle\omega_{\hbar} ; W^{\hbar}(f)\right\rangle=0$ for each $f \in E$. For $\hbar_{0} \neq 0$ and $\mu \in M^{\tau}(E)$ we conclude that

$$
\begin{aligned}
& \left\langle\omega_{\hbar} ; Q_{\hbar}^{\Pi}(\widehat{\mu})^{*} Q_{\hbar}^{\Pi}(\widehat{\mu})\right\rangle=\int_{E} d \mu^{*}(f) \int_{E} d \mu(g) \exp \left\{-\frac{i}{2} \hbar \sigma(f, g)\right\}\left\langle\omega_{\hbar} ; W^{\hbar}(f+g)\right\rangle \\
& \stackrel{\hbar \rightarrow \hbar_{0}}{\longrightarrow} \int_{E} d \mu^{*}(f) \int_{E} d \mu(g) \exp \left\{-\frac{i}{2} \hbar_{0} \sigma(f, g)\right\}\left\langle\omega_{\hbar_{0}} ; W^{\hbar_{0}}(f+g)\right\rangle=\left\langle\omega_{\hbar_{0}} ; Q_{\hbar_{0}}^{\Pi}(\widehat{\mu})^{*} Q_{\hbar_{0}}^{\Pi}(\widehat{\mu})\right\rangle .
\end{aligned}
$$

Consequently $\left\langle\omega_{\hbar_{0}} ; Q_{\hbar_{0}}^{\Pi}(\widehat{\mu})^{*} Q_{\hbar_{0}}^{\Pi}(\widehat{\mu})\right\rangle=\lim _{\hbar \rightarrow \hbar_{0}}\left\langle\omega_{\hbar} ; Q_{\hbar}^{\Pi}(\widehat{\mu})^{*} Q_{\hbar}^{\Pi}(\widehat{\mu})\right\rangle \leq \liminf _{\hbar \rightarrow \hbar_{0}}\left\|Q_{\hbar}^{\Pi}(\widehat{\mu})\right\|^{2}$. Since $\mathcal{F}_{\hbar}$ consists of the normal states on ${\overline{\Pi_{\hbar}(\mathcal{W}(E, \hbar \sigma))}}^{w}$, we have $\|A\|^{2}=\sup \left\{\left\langle\varphi ; A^{*} A\right\rangle \mid \varphi \in \mathcal{F}_{\hbar}\right\}$ for all $A \in{\overline{\Pi_{\hbar}(\mathcal{W}(E, \hbar \sigma))}}^{w}$. Hence, taking the supremum over all states $\omega \in \mathcal{F}_{1}$, or equivalently over the states $\omega_{\hbar_{0}} \in \mathcal{F}_{\hbar_{0}}$, we conclude the lower semicontinuity at $\hbar_{0} \neq 0$, more precisely

$$
\left\|Q_{\hbar_{0}}^{\Pi}(\widehat{\mu})\right\| \leq \liminf _{\hbar \rightarrow \hbar_{0}}\left\|Q_{\hbar}^{\Pi}(\widehat{\mu})\right\| \leq \limsup _{\hbar \rightarrow \hbar_{0}}\left\|Q_{\hbar}^{\Pi}(\widehat{\mu})\right\| \leq\|\mu\|_{1}, \quad \forall \mu \in M^{\tau}(E) .
$$

Similarly we obtain for $\hbar_{0}=0$ that

$$
\left\langle\omega_{\hbar} ; Q_{\hbar}^{\Pi}(\widehat{\mu})^{*} Q_{\hbar}^{\Pi}(\widehat{\mu})\right\rangle \stackrel{\hbar \rightarrow 0}{\longrightarrow} \int_{E} d \mu^{*}(f) \int_{E} d \mu(g)=\widehat{\mu}^{*}[0] \widehat{\mu}[0]=|\widehat{\mu}[0]|^{2},
$$

and hence $|\widehat{\mu}[0]| \leq \liminf _{\hbar \rightarrow 0}\left\|Q_{\hbar}^{\Pi}(\widehat{\mu})\right\| \leq \limsup _{\hbar \rightarrow 0}\left\|Q_{\hbar}^{\Pi}(\widehat{\mu})\right\| \leq\|\mu\|_{1}$ for all $\mu \in M^{\tau}(E)$. But Lemma 7 tells that $\|\mu\|_{1}=\widehat{\mu}[0]$ for all $\mu \in M^{\tau}(E)^{+}$.

Suppose now the additional property of (partial) $E_{\rho}^{\prime}$-invariance. Then there exists a state $\varphi \in \mathcal{F}_{1}$ so that $\varphi_{\hbar}^{F}:=\beta_{\hbar}^{*}(\varphi) \circ \gamma_{F}^{\hbar} \in \mathcal{F}_{\hbar}$ for all $F \in E_{\rho}^{\prime}$ for each $\hbar \neq 0$. We have $\left\langle\varphi_{\hbar}^{F} ; W^{\hbar}(f)\right\rangle=$ $\exp \{i F(f)\}\left\langle\varphi ; W^{1}\left(T_{\hbar} f\right)\right\rangle$ for each $f \in E$. Similarly to the above reasoning we get

$$
\begin{aligned}
\left\langle\varphi_{\hbar}^{F} ;\right. & \left.Q_{\hbar}^{\Pi}(\widehat{\mu})^{*} Q_{\hbar}^{\Pi}(\widehat{\mu})\right\rangle \\
& =\int_{E} d \mu^{*}(f) \int_{E} d \mu(g) \exp \left\{i\left(F(f)+F(g)-\frac{1}{2} \hbar \sigma(f, g)\right)\right\}\left\langle\varphi ; W^{1}\left(T_{\hbar}(f+g)\right)\right\rangle \\
& \stackrel{\hbar \rightarrow 0}{\longrightarrow} \int_{E} d \mu^{*}(f) \int_{E} d \mu(g) \exp \{i F(f)\} \exp \{i F(g)\}=\widehat{\mu}^{*}[F] \widehat{\mu}[F]=|\widehat{\mu}[F]|^{2}
\end{aligned}
$$

for all $F \in E_{\rho}^{\prime}$. Taking the sup-norm for $\widehat{\mu}$ yields $\|\widehat{\mu}\|_{0} \leq \liminf _{\hbar \rightarrow 0}\left\|Q_{\hbar}^{\Pi}(\widehat{\mu})\right\|$ for all $\mu \in M^{\tau}(E)$.

The proof of Proposition 4 now is easy. For non-degenerate $\sigma$ it follows from Lemma 6(b) that the well matching properties are not necessary, since the norms for regular families $\Pi$ don't differ from those of the well matched families $\Pi^{\tau}$, resp. $\Pi^{\text {reg }}$. The result follows with help of the previous proof.

\section{References}

[1] Emch G.G., Algebraic methods in statistical mechanics and quantum field theory, John Wiley and Sons, New York, 1972.

[2] Bratteli O., Robinson D.W., Operator algebras and quantum statistical mechanics, Vol. II, Springer, New York, 1981.

[3] Reed S., Simon B., Fourier analysis, self-adjointness, Vol. II, Academic Press, New York, 1975. 
[4] Weyl H., Quantenmechanik und Gruppentheorie, Z. Phys. 46 (1928), 1-46.

[5] Weyl H., The theory of groups and quantum mechanics, Methuen, London, 1931 (reprinted by Dover Publ., New York, 1950).

[6] Putnam C.R., Commutation properties of Hilbert space operators and related topics, Springer, New York Berlin - Heidelberg, 1967.

[7] Galindo A., Pascual P., Quantum mechanics, Vols. I, II, Springer, New York - Berlin, 1989, 1991.

[8] Honegger R., On Heisenberg's uncertainty principle and the CCR, Z. Naturforsch. A 48 (1993), $447-451$.

[9] von Neumann J., Die Eindeutigkeit der Schrödingerschen Operatoren, Math. Ann. 104 (1931), $570-578$.

[10] Rieffel M.A., Quantization and $C^{*}$-algebras, in " $C^{*}$-Algebras: 1943-1993", Editor R.S. Doran, Contemp. Math. 167 (1994), 67-97.

[11] Honegger R., Rieckers A., Some continuous field quantizations, equivalent to the $C^{*}$-Weyl quantization, Publ. RIMS Kyoto Univ. 41 (2005), 113-138.

[12] Garcia-Bondía J.M., Varilly C., Algebras of distributions suitable for phase-space quantum mechanics I, J. Math. Phys. 29 (1988), 869-879.

[13] Kastler D., The $C^{*}$-algebras of a free Boson field, Comm. Math. Phys. 1 (1965), 14-48.

[14] Binz E., Honegger R., Rieckers A., Field-theoretic Weyl quantization as a strict and continuous deformation quantization, Ann. Henri Poincaré 5 (2004), 327-346.

[15] Loudon R., The quantum theory of light, Clarendon Press, Oxford, 1979.

[16] Cohen-Tannoudji C., Dupont-Roc J., Grynberg G., Photons \& atoms, introduction to QED, John Wiley \& Sons, New York - Toronto - Singapore, 1989.

[17] Schwarz G., Hodge decompositions - a method for solving boundary value problems, Lecture Notes in Mathematics, Vol. 1607, Springer, Berlin - New York, 1995.

[18] Buchholz D., Mack G., Todorov I., The current algebra on the circle as a germ of the local field theories, Nuclear Phys. B 5 (1988), 20-56.

[19] Bayen F., Flato M., Fronsdal C., Lichnerovicz A., Sternheimer D., Deformation theory and quantization, Ann. Phys. 111 (1978), 61-151.

[20] Dito J., Star-products and nonstandard quantization for the Klein-Gordon equation, J. Math. Phys. 33 (1992), 791-801.

[21] Dito J., An example of cancellation of infinities in the star-product quantization of fields, Lett. Math. Phys. 27 (1993), 73-80.

[22] Dütsch M., Fredenhagen K., Algebraic quantum field theory, perturbation theory, and the loop expansion, Comm. Math. Phys. 219 (2001), 5-30, hep-th/0001129.

[23] Sternheimer D., Alcade C.A., Analytic vectors, anomalies and star representations, Lett. Math. Phys. 17 (1989), 117-127.

[24] Fronsdal C., Normal ordering and quantum groups, Lett. Math. Phys. 22 (1991), 225-228.

[25] Dito J., Star-product approach to quantum field theory: the free scalar field, Lett. Math. Phys. 20 (1990), $125-134$.

[26] Rieffel M.A., Deformation quantization for actions of $\mathbb{R}^{d}$, Mem. Amer. Math. Soc. 106 (1993), no. 506.

[27] Rieffel M.A., Questions on quantization, in Operator Algebras and Operator Theory (1997, Shanghai), Contemp. Math. 228 (1998), 315-326, quant-ph/9712009.

[28] Landsman N.P., Strict quantization of coadjoint orbits, J. Math. Phys. 39 (1998), 6372-6383, math-ph/9807027.

[29] Landsman N.P., Mathematical topics between classical and quantum mechanics, Springer, New York, 1998.

[30] Manuceau J., Sirugue M., Testard D., Verbeure A., The smallest $C^{*}$-algebra for canonical commutation relations, Comm. Math. Phys. 32 (1973), 231-243.

[31] Binz E., Honegger R., Rieckers A., Construction and uniqueness of the $C^{*}$-Weyl algebra over a general pre-symplectic form, J. Math. Phys. 45 (2004), 2885-2907.

[32] Hewitt E., Ross K.A., Abstract harmonic analysis, Vols. I, II, Springer, New York, 1963, 1970.

[33] Abraham R., Marsden J.E., Foundations of mechanics, 2nd ed., Benjamin/Cummings Publishing Company, London, Amsterdam, 1978. 
[34] Arnold V.I., Mathematical methods of classical mechanics, Springer, New York, 1985.

[35] Libermann P., Marle C.-M., Symplectic geometry and analytical mechanics, D. Reidel Publ. Co, Dordrecht, 1987.

[36] Binz E., Sniatycki J., Fischer H., Geometry of classical fields, Mathematics Studies, Vol. 154, North Holland, Amsterdam, 1988.

[37] Marsden J.E., Ratiu T., Introduction to mechanics and symmetry, Springer, New York, Berlin, 1994.

[38] Schaefer H.H., Topological vector spaces, Macmillan Company, New York, 1966.

[39] Conway J.B., A course in functional analysis, Springer, New York, 1985.

[40] Cohn D.L., Measure theory, Birkhäuser, Boston, 1980.

[41] Edwards C.M., Lewis J.T., Twisted group algebras I, II, Comm. Math. Phys. 13 (1969), 119-141.

[42] Busby R.C., Smith H.A., Representations of twisted group algebras, Trans. Amer. Math. Soc. 149 (1970), 503-537.

[43] Dixmier J., $C^{*}$-algebras, North-Holland, Amsterdam, 1977.

[44] Pedersen G.K., $C^{*}$-algebras and their automorphism groups, Academic Press, London, 1979.

[45] Packer J.A., Raeburn I., Twisted crossed products of $C^{*}$-algebras, Math. Proc. Camb. Phil. Soc. 106 (1989), 293-311.

[46] Grundling H., A group algebra for inductive limit groups, continuity problems of the canonical commutation relations, Acta Appl. Math. 46 (1997), 107-145.

[47] Haag R., Kadison R.V., Kastler D., Nets of $C^{*}$-algebras and classification of states, Comm. Math. Phys. 16 (1970), 81-104.

[48] Sewell G.L., States and dynamics of infinitely extended physical systems, Comm. Math. Phys. 33 (1973), $43-51$.

[49] Kadison R.V., Ringrose J.R., Fundamentals of the theory of operator algebras, Vols. I, II, Academic Press, New York, 1983, 1986.

[50] Honegger R., Global cocycle dynamics for infinite mean field quantum systems interacting with the Boson gas, J. Math. Phys. 37 (1996), 263-282.

[51] Bratteli O., Robinson D.W., Operator algebras and quantum statistical mechanics, Vol. I, Springer, New York, 1987.

[52] Honegger R., Rieckers A., Partially classical states of a Boson field, Lett. Math. Phys. 64 (2003), 31-44.

[53] Robinson P.L., Symplectic pathology, Quart. J. Math. Oxford 44 (1993), 101-107.

[54] Honegger R., On the continuous extension of states on the CCR-algebra, Lett. Math. Phys. 42 (1997), 11-25.

[55] Honegger R., Enlarged testfunction spaces for the global free folia dynamics on the CCR-algebra, J. Math. Phys. 39 (1998), 1153-1169.

[56] Segal I.E., Distributions in Hilbert space and canonical systems of operators, Trans. Amer. Math. Soc. 88 (1958), 12-41.

[57] Hörmann G., Regular Weyl-systems and smooth structures on Heisenberg groups, Comm. Math. Phys. 184 (1997), 51-63.

[58] Segal I.E., Representations of the canonical commutation relations, in Cargese Lectures in Theoretical Physics, Gordon and Breach, 1967, 107-170.

[59] Folland G., Harmonic analysis in phase space, Annals of Mathematics Studies, Vol. 122, Princeton University Press, Princeton, 1989.

[60] Fell J.M.G., Doran R.S. Representations of *-algebras, locally compact groups, and Banach *-algebraic bundles, Pure and Applied Mathematics, Vol. 126, Academic Press, New York, London, 1988.

[61] Takesaki M., Theory of operator algebras, Vol. I, Springer, New York, 1979. 